\title{
Ir-Catalyzed C-H Amidation using Carbamoyl Azides for the Syntheses of Unsymmetrical Ureas
}

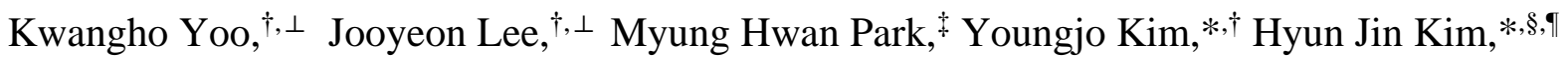
and Min Kim*, ${ }^{*}$

${ }^{\dagger}$ Department of Chemistry, Chungbuk National University, Cheongju, Korea 28644

${ }^{\ddagger}$ Department of Chemistry Education, Chungbuk National University, Cheongju, Korea 28644

${ }^{\S}$ Innovative Therapeutic Research Center, Therapeutics and Biotechnology Division, Korea Research Institute of Chemical Technology (KRICT), Daejeon, Korea 34114

"Department of New Drug Discovery and Development, Chungnam National University, Daejeon, Korea 34134

*E-mail: ykim@chungbuk.ac.kr (Y.K.), hyunjin@krict.re.kr (H.J.K.), and minkim@chungbuk.ac.kr (M.K)

\section{Contents}

I. Additional Optimization Studies

II. Additional Directing Group Tests

III. Proposed Mechanism for C-H Amidation with Carbamoyl Azide

\section{Appendix I}

Spectral Copies of ${ }^{1} \mathrm{H},{ }^{13} \mathrm{C}$-NMR and FT-IR of Compounds Obtained in this Study 


\section{Additional Optimization Studies}

$N$-(tert-Butyl)benzamide (1a, $27 \mathrm{mg}, 0.15 \mathrm{mmol}$ ), diphenylcarbamoyl azide (2c, $24 \mathrm{mg}, 0.1 \mathrm{mmol}$ ), $\left[\mathrm{IrCp}^{*} \mathrm{Cl}_{2}\right]_{2}(4 \mathrm{mg}, 0.005 \mathrm{mmol}), \operatorname{AgNTf}_{2}(4 \mathrm{mg}, 0.01 \mathrm{mmol})$ and additive were dissolved in solvent $(0.35 \mathrm{~mL})$ at a screw capped vial with spinvane type triangular-shaped stir bar. The reaction mixture was stirred at $35^{\circ} \mathrm{C}$ for $12 \mathrm{~h}$. After completion, the mixture was filtered through a silica gel and then washed with EtOAc (3 $\mathrm{mL}$ x 3). Organic solvents were removed under reduced pressure and the crude yield was measured by ${ }^{1} \mathrm{H}$ NMR using dibromomethane as an internal standard.

Table S1. Additional solvent and additive screening for optimization ${ }^{a}$

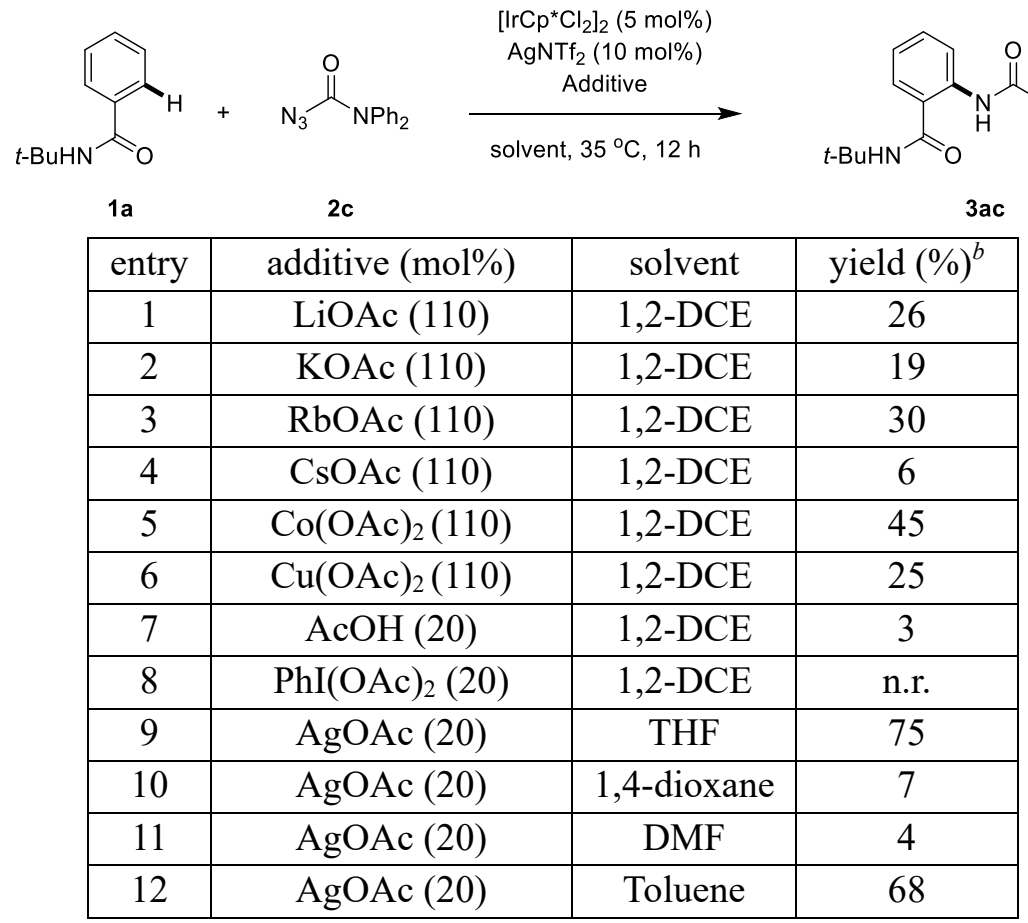

${ }^{a}$ Reaction condition: 1a $(0.15 \mathrm{mmol}, 27 \mathrm{mg}), \mathbf{2 c}(0.1 \mathrm{mmol}, 24 \mathrm{mg}),\left[\mathrm{IrCp}^{*} \mathrm{Cl}_{2}\right]_{2}(5 \mathrm{~mol} \%), \operatorname{AgNTf}_{2}(10$ mol\%), and additives in solvent $(0.35 \mathrm{~mL})$ for $12 \mathrm{~h}$ at $35{ }^{\circ} \mathrm{C} .{ }^{b}$ Yield based on ${ }^{1} \mathrm{H}$ NMR analysis of the crude reaction mixture using $\mathrm{CH}_{2} \mathrm{Br}_{2}$ as the internal standard. 
Table S2. Additional substrate ratio screening for optimization ${ }^{a}$

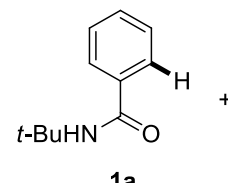

$1 a$

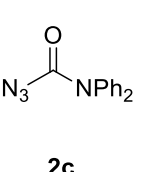

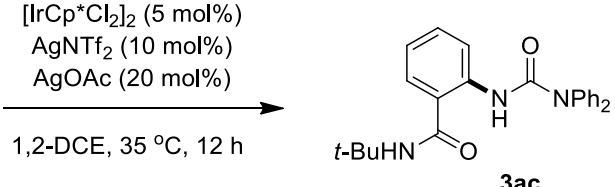

3ac

\begin{tabular}{|c|c|c|}
\hline entry & 1a:2c & ${\text { yield }(\%)^{b}}^{b}$ \\
\hline 1 & $1: 1$ & 82 \\
\hline 2 & $1.2: 1$ & 83 \\
\hline 3 & $1.5: 1$ & 93 \\
\hline 4 & $1: 1.2$ & 82 \\
\hline
\end{tabular}

${ }^{a}$ Reaction conditions: $1 \mathrm{a}(0.15 \mathrm{mmol}, 27 \mathrm{mg}), \mathbf{2 c}(0.1 \mathrm{mmol}, 24 \mathrm{mg}),\left[\mathrm{IrCp}^{*} \mathrm{Cl}_{2}\right]_{2}(5 \mathrm{~mol} \%, 4 \mathrm{mg})$, $\operatorname{AgNTf}_{2}(10 \mathrm{~mol} \%, 4 \mathrm{mg})$, and $\operatorname{AgOAc}(20 \mathrm{~mol} \%, 3 \mathrm{mg})$ in 1,2-DCE $(0.35 \mathrm{~mL})$ for $12 \mathrm{~h}$ at $35{ }^{\circ} \mathrm{C}$. Isolated yields from an average of two independent experiments. 


\section{Additional Directing Group Tests}

Directing group derivatives $(0.15 \mathrm{mmol})$, diphenylcarbamoyl azide (2c, $24 \mathrm{mg}, 0.1 \mathrm{mmol})$, $\left[\mathrm{IrCp} * \mathrm{Cl}_{2}\right]_{2}$ (4 mg, $0.005 \mathrm{mmol}), \operatorname{AgNTf}_{2}(4 \mathrm{mg}, 0.01 \mathrm{mmol}$ ) and AgOAc (3 mg, $0.02 \mathrm{mmol}$ ) were dissolved in 1,2dichloroethane $(0.35 \mathrm{~mL})$ at a screw capped vial with spinvane type triangular-shaped stir bar. The reaction mixture was stirred at $35^{\circ} \mathrm{C}$ for $12 \mathrm{~h}$. After completion, the mixture was filtered through a silica gel and then washed with EtOAc ( $3 \mathrm{~mL} x 3)$, and analyzed by TLC.

Table S3. Additional directing group test for optimization ${ }^{a}$

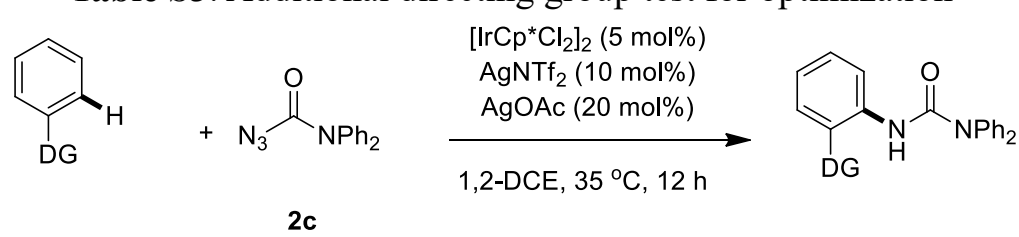

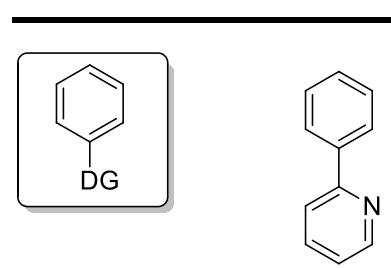

n.r.<smiles>NC(=O)Oc1ccccc1</smiles>

n.r.<smiles>O=C(c1ccccc1)N1CCCCC1</smiles>

trace

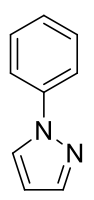

n.r.

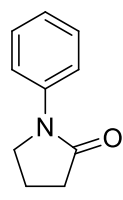

n.r.<smiles>CON=Cc1ccc(Cl)cc1</smiles>

n.r.<smiles>c1ccc(-c2cocn2)cc1</smiles>

n.r.<smiles>CC(=O)c1ccccc1</smiles><smiles>CC(C)(C)OC(=O)c1ccccc1</smiles>

trace

trace<smiles>CC(=N)c1ccccc1</smiles>

n.r.

${ }^{a}$ Reaction condition: $1(0.15 \mathrm{mmol}), \mathbf{2 c}(0.1 \mathrm{mmol}, 24 \mathrm{mg}),\left[\mathrm{IrCp}^{*} \mathrm{Cl}_{2}\right]_{2}(5 \mathrm{~mol} \%), \operatorname{AgNTf}_{2}(10 \mathrm{~mol} \%)$, and $\mathrm{AgOAc}(20 \mathrm{~mol} \%)$ in $1,2-\mathrm{DCE}(0.35 \mathrm{~mL})$ for $12 \mathrm{~h}$ at $35^{\circ} \mathrm{C} .{ }^{b}$ n.r. $=$ no reaction. 


\section{Proposed Mechanism for C-H Amidation with Carbamoyl Azide}

Scheme S1. Plausible Mechanism for Ir-Catalyzed C-H Amidation with Carbamoyl Azide.

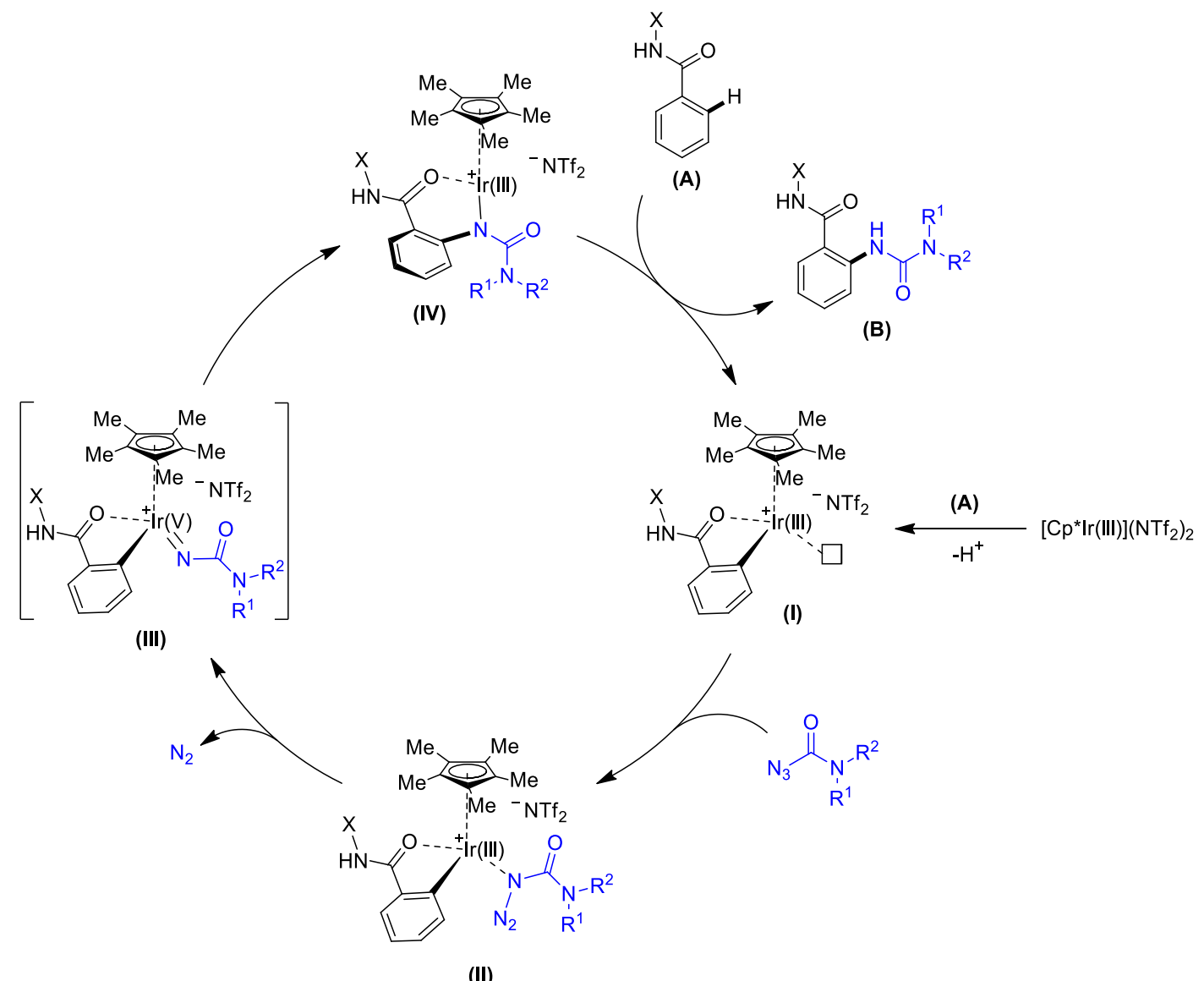




\section{Appendix I}

\section{Spectral Copies of ${ }^{1} \mathrm{H}$ and ${ }^{13} \mathrm{C}$ NMR and FT-IR Spectra of the Obtained Compounds in this Study}


Di-p-tolylcarbamoyl azide (Table 2, 2d, ${ }^{1} \mathrm{H}$ NMR-500 MHz \& ${ }^{13} \mathrm{C}$ NMR-125 MHz in $\mathrm{CDCl}_{3}$ )
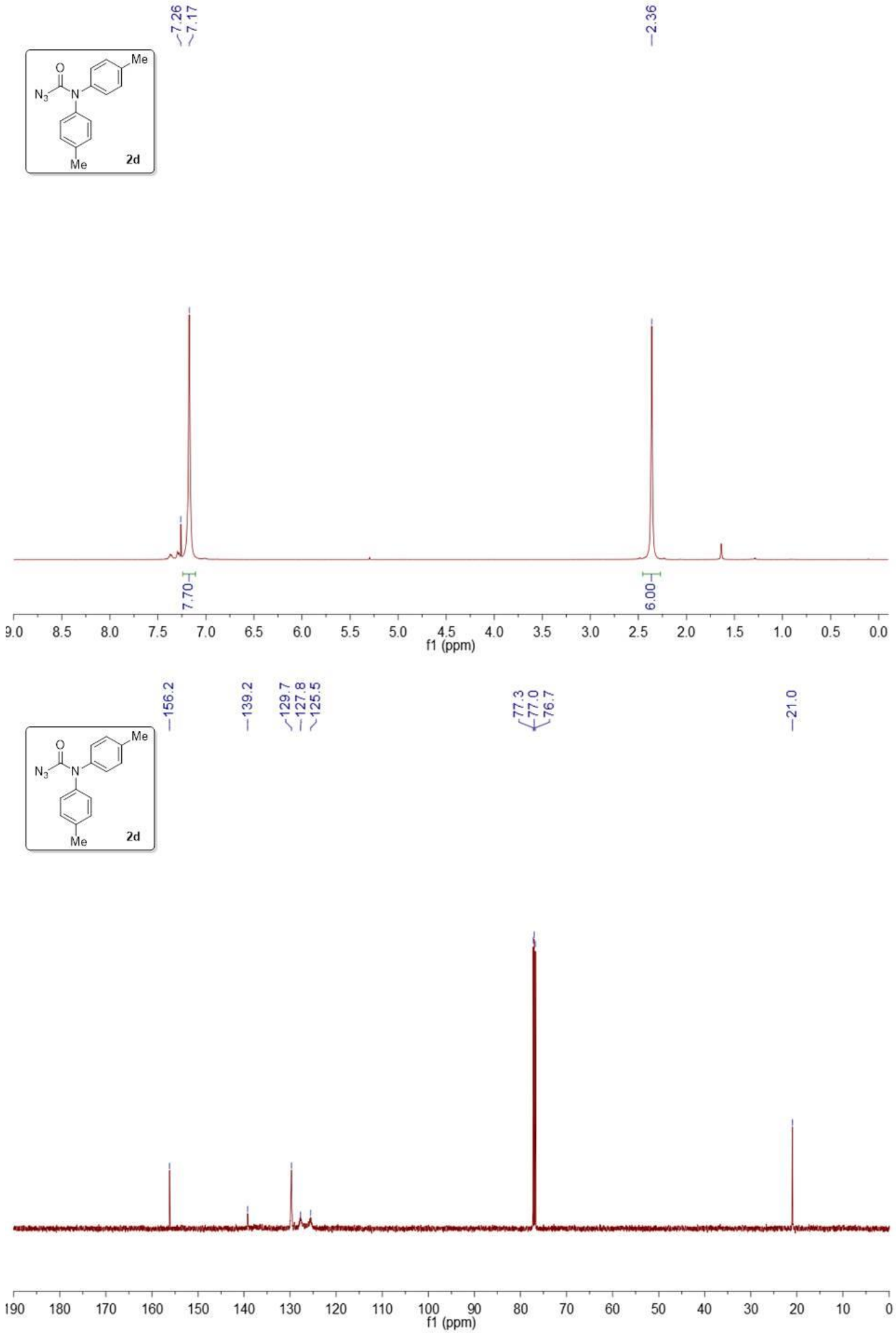


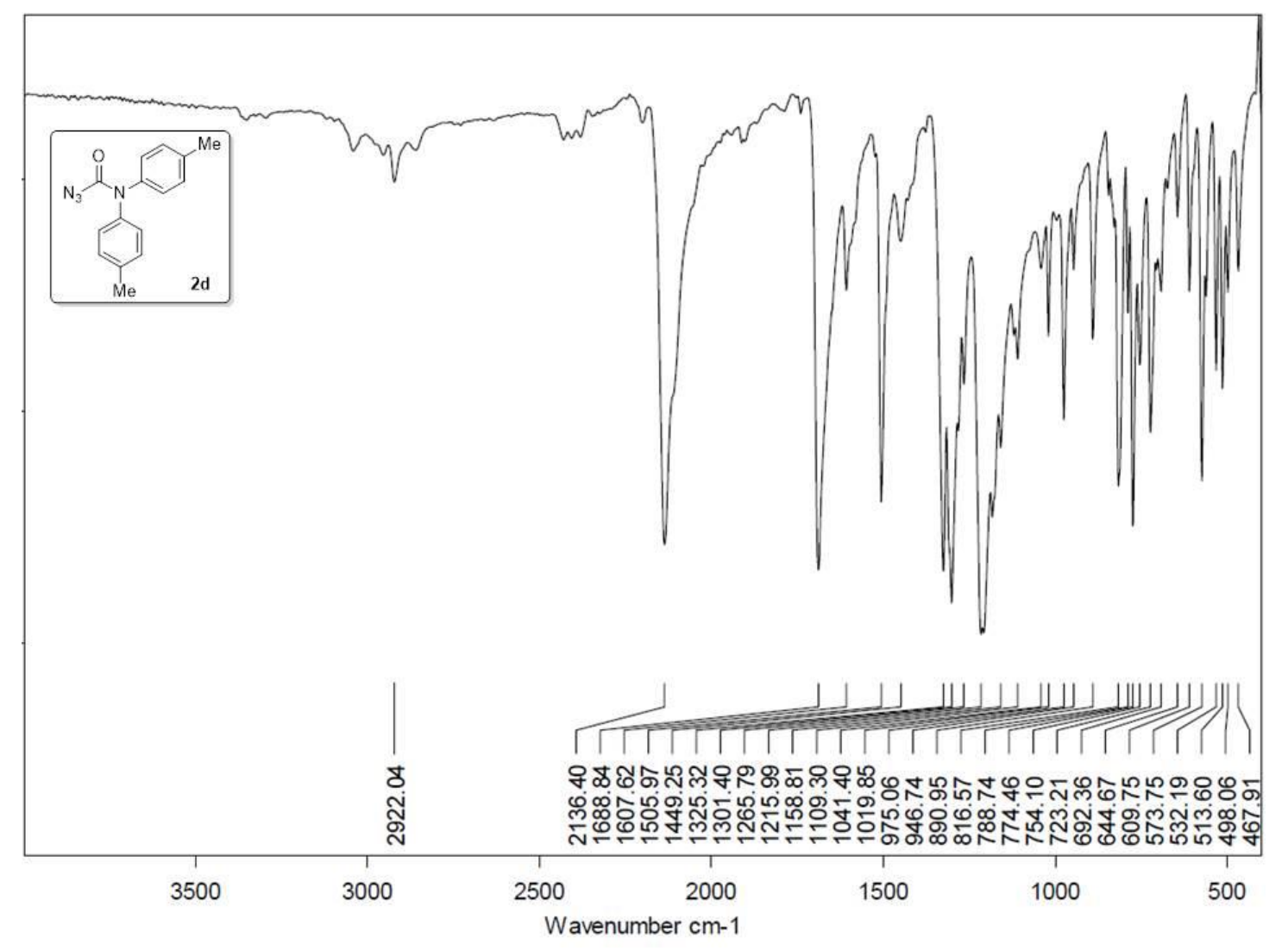


(4-(tert-Butyl)phenyl)(p-tolyl)carbamoyl azide (Table 2, 2e, ${ }^{1} \mathrm{H}$ NMR-500 MHz \& ${ }^{13} \mathrm{C}$ NMR-125 $\mathrm{MHz}$ in $\mathrm{CDCl}_{3}$ )
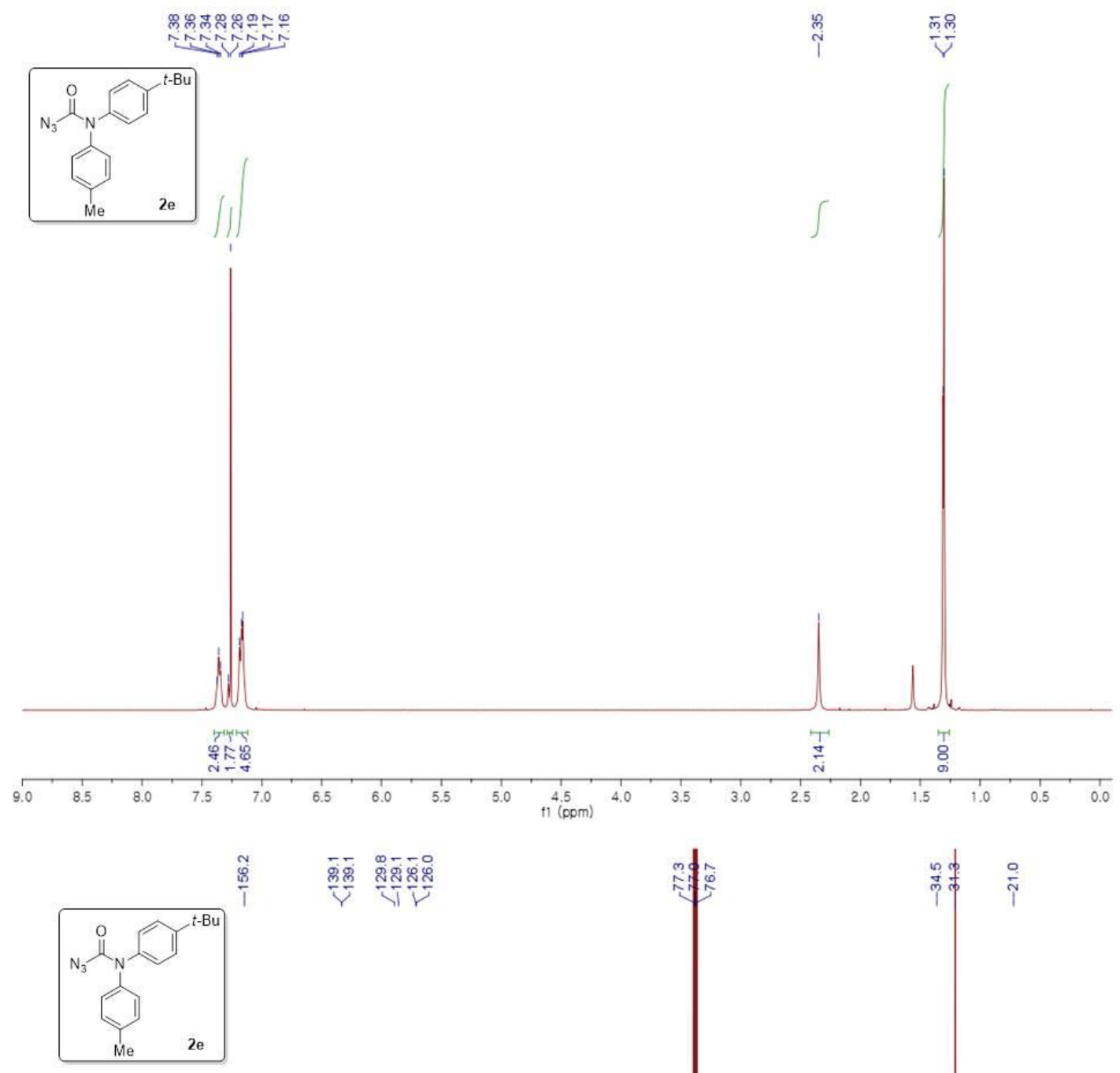

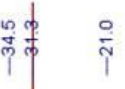

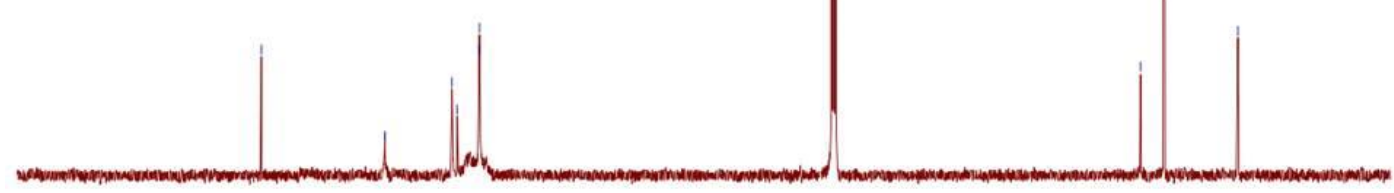

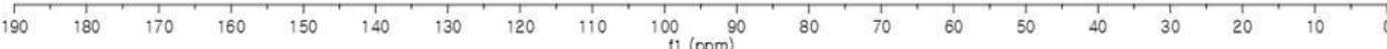




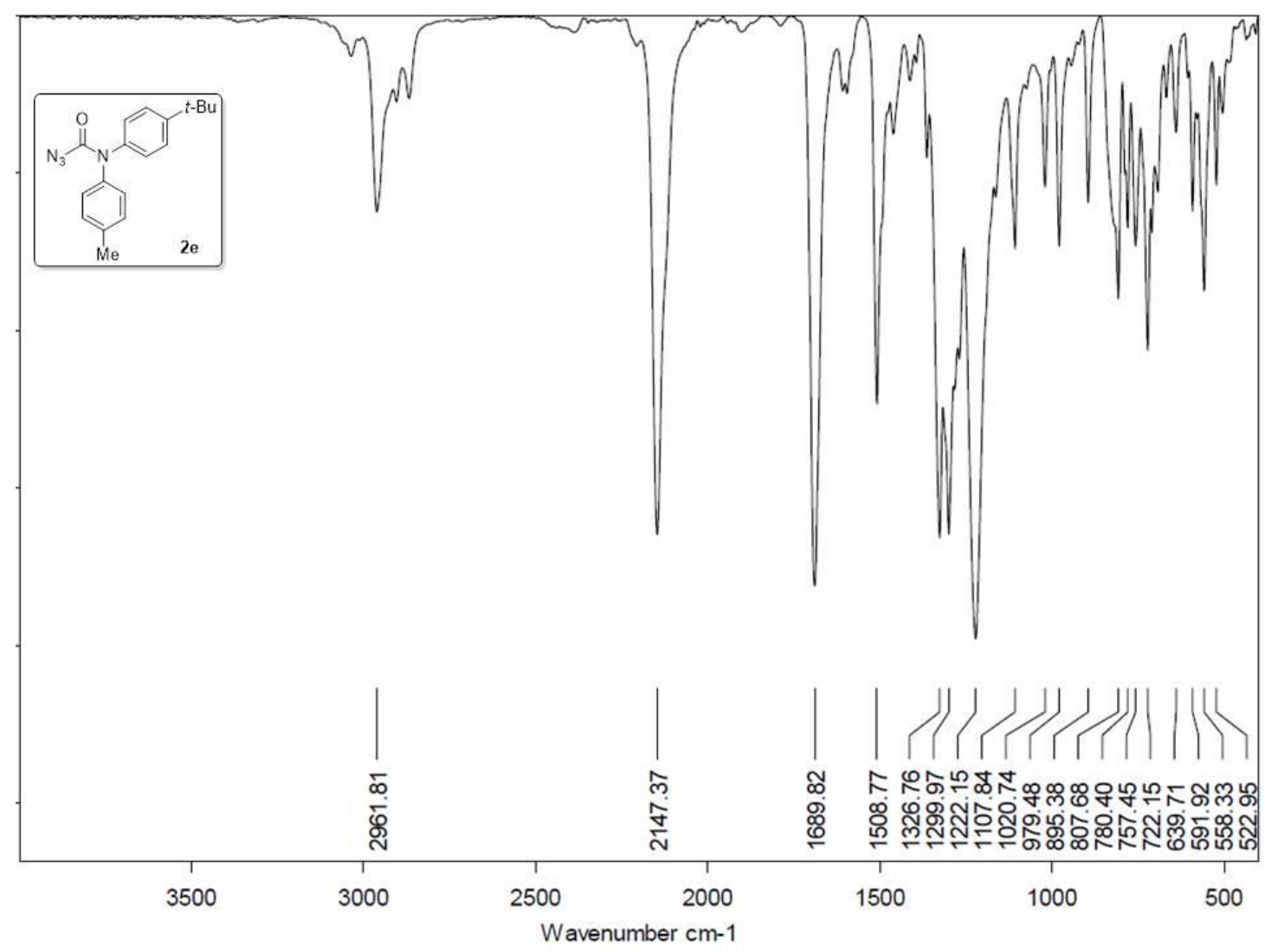


(4-Chlorophenyl)(4-fluorophenyl)carbamoyl azide (Table 2, 2f, ${ }^{1} \mathrm{H}$ NMR-500 MHz \& ${ }^{13} \mathrm{C}$ NMR$125 \mathrm{MHz}$ in $\mathrm{CDCl}_{3}$ )
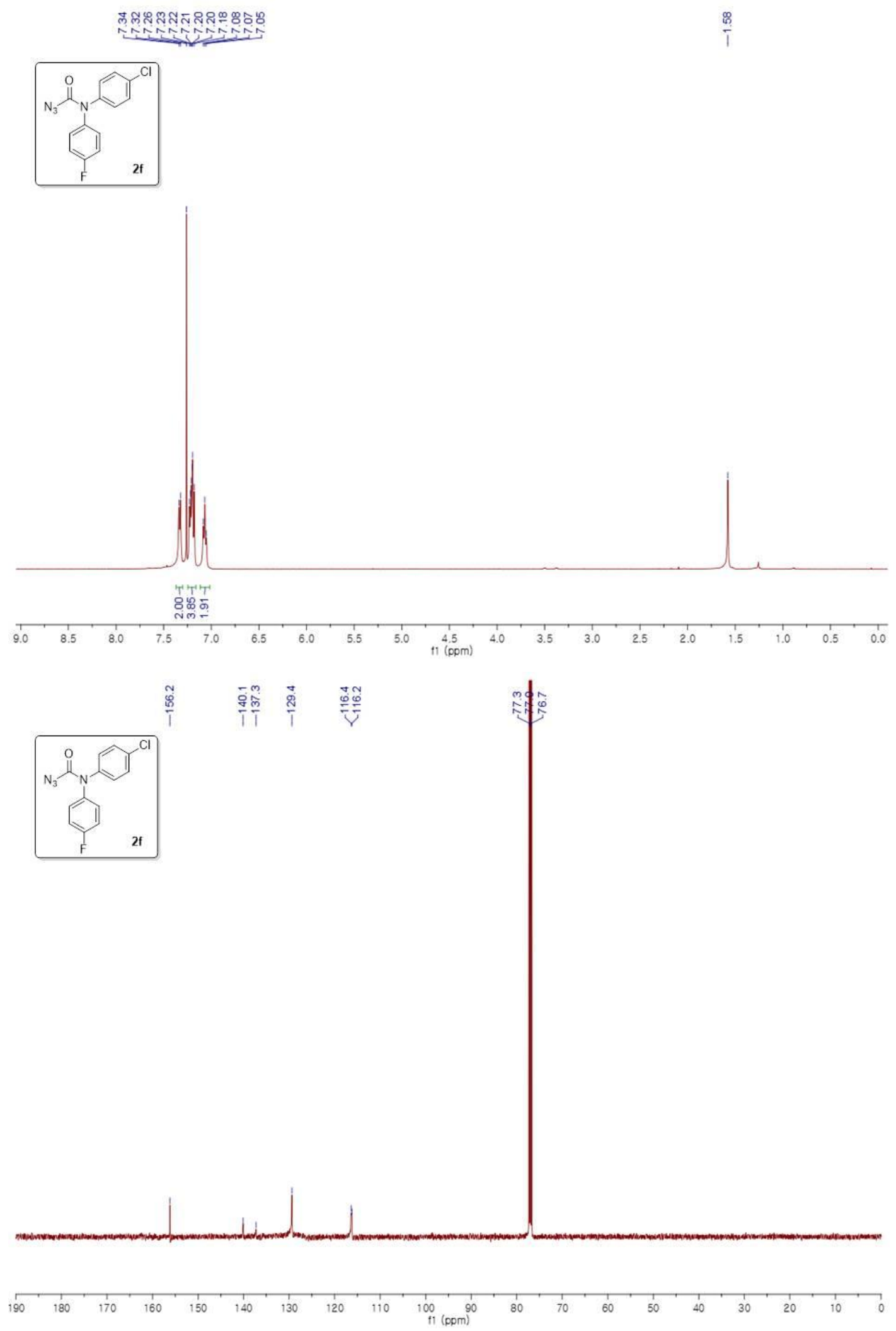


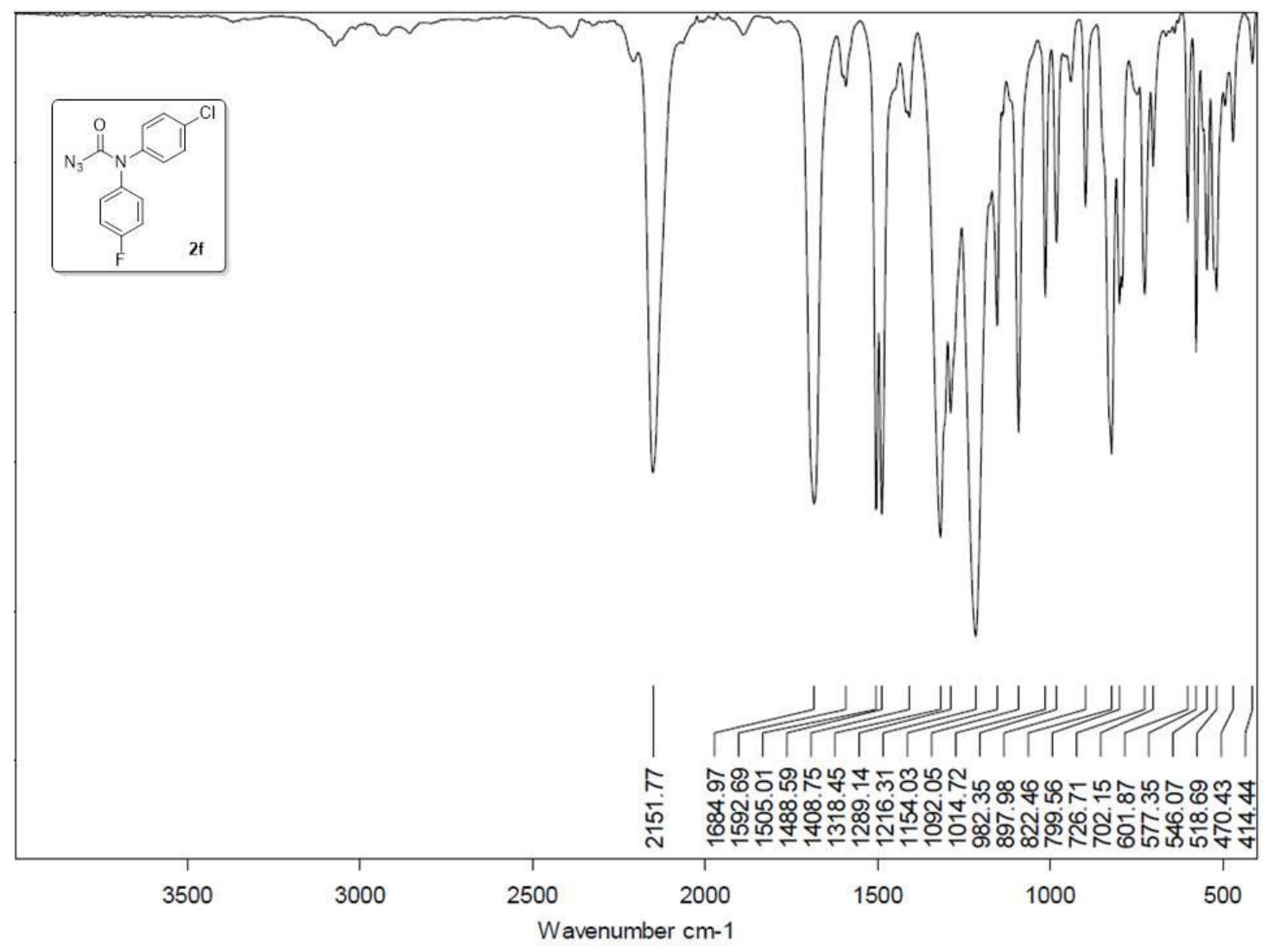


(4-Fluoro-2-methylphenyl)(4-fluorophenyl)carbamoyl azide (Table 2, 2g, ${ }^{1} \mathrm{H}$ NMR-500 MHz \& ${ }^{13} \mathrm{C}$ NMR-125 MHz in $500 \mathrm{MHz}, \mathrm{CDCl}_{3}$ )
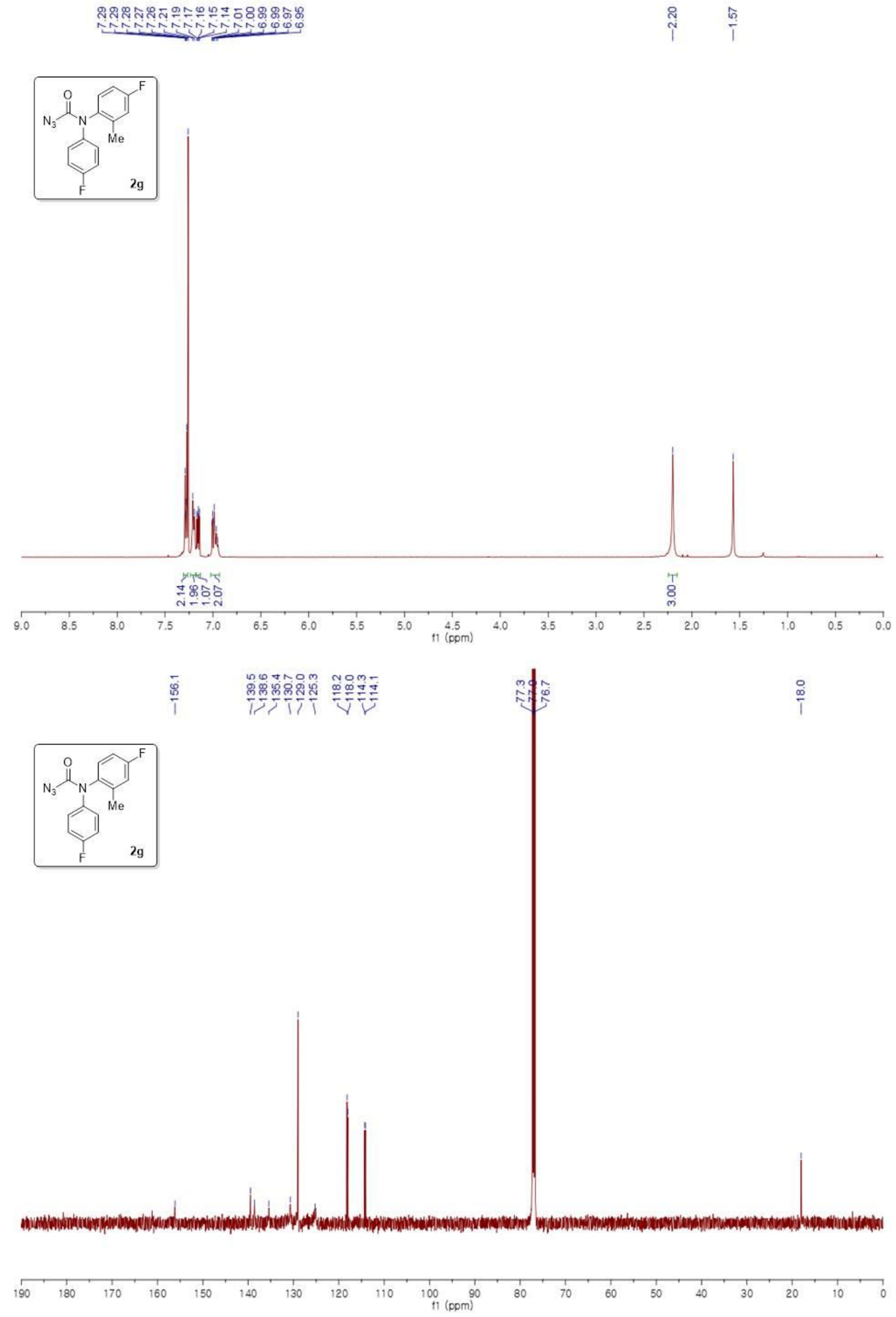


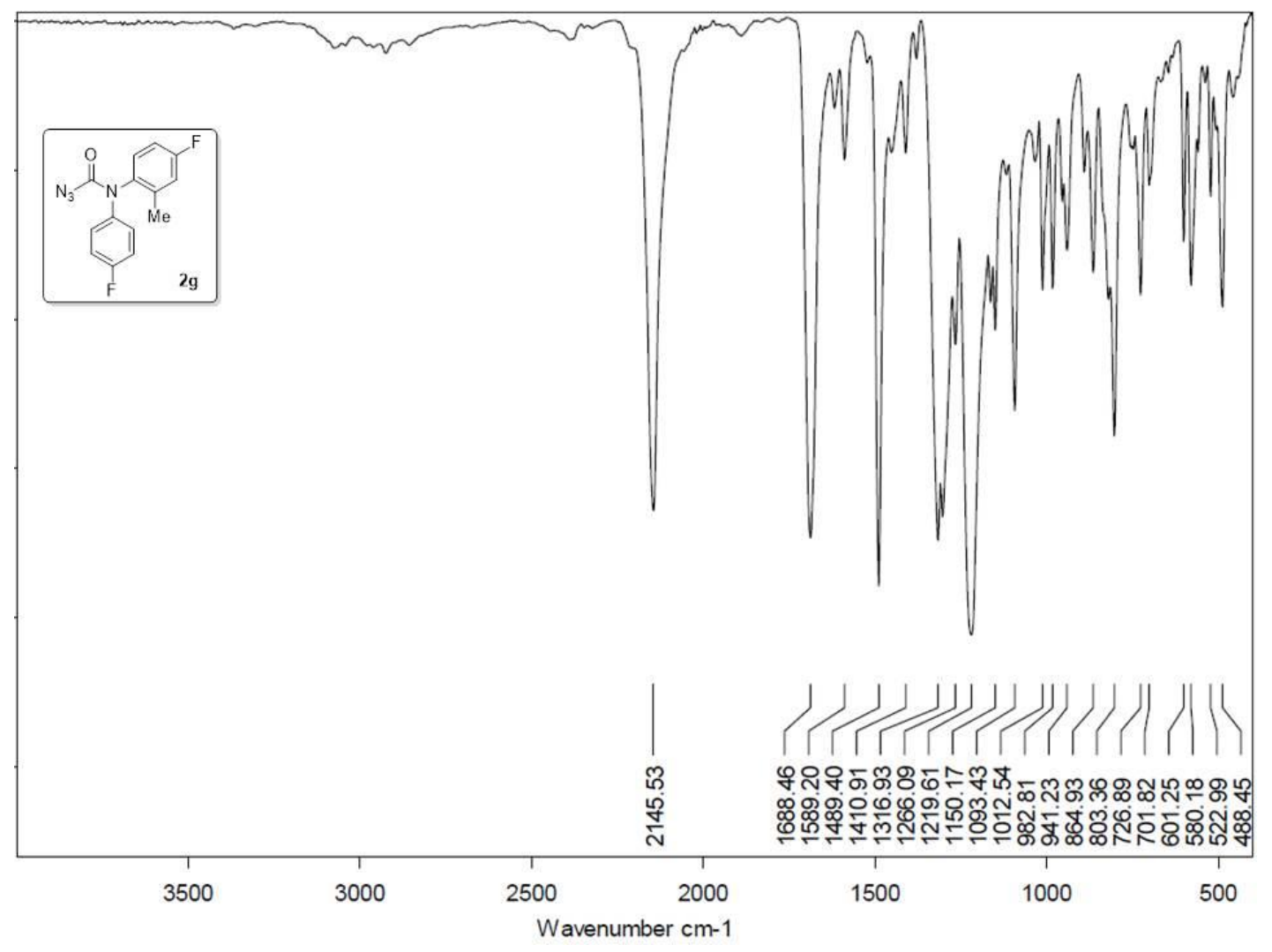


Methyl(phenyl)carbamoyl azide (Table 2, 2i, ${ }^{1} \mathrm{H}$ NMR-500 $\mathrm{MHz} \&{ }^{13} \mathrm{C}$ NMR-125 MHz in $\mathrm{CDCl}_{3}$ )
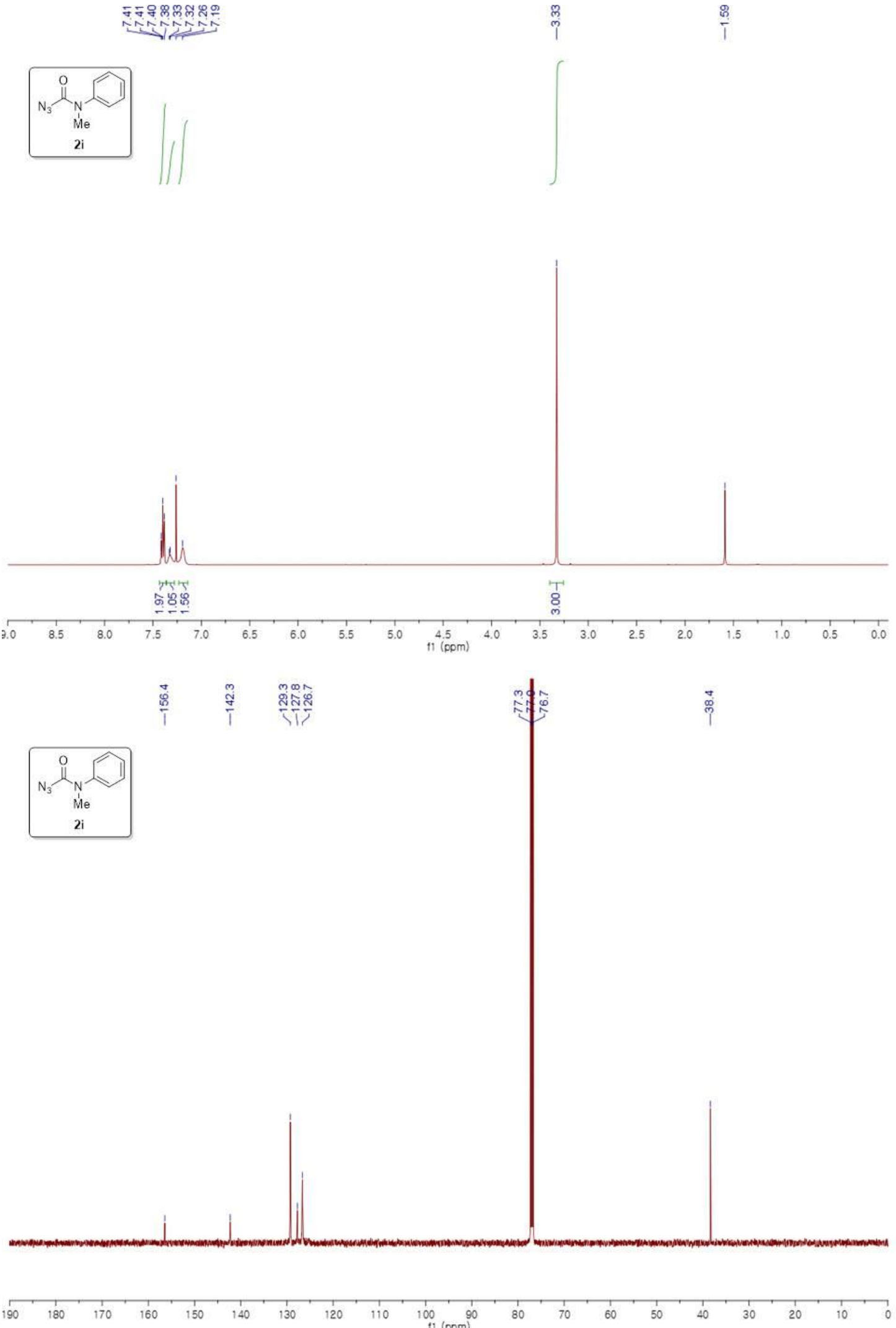


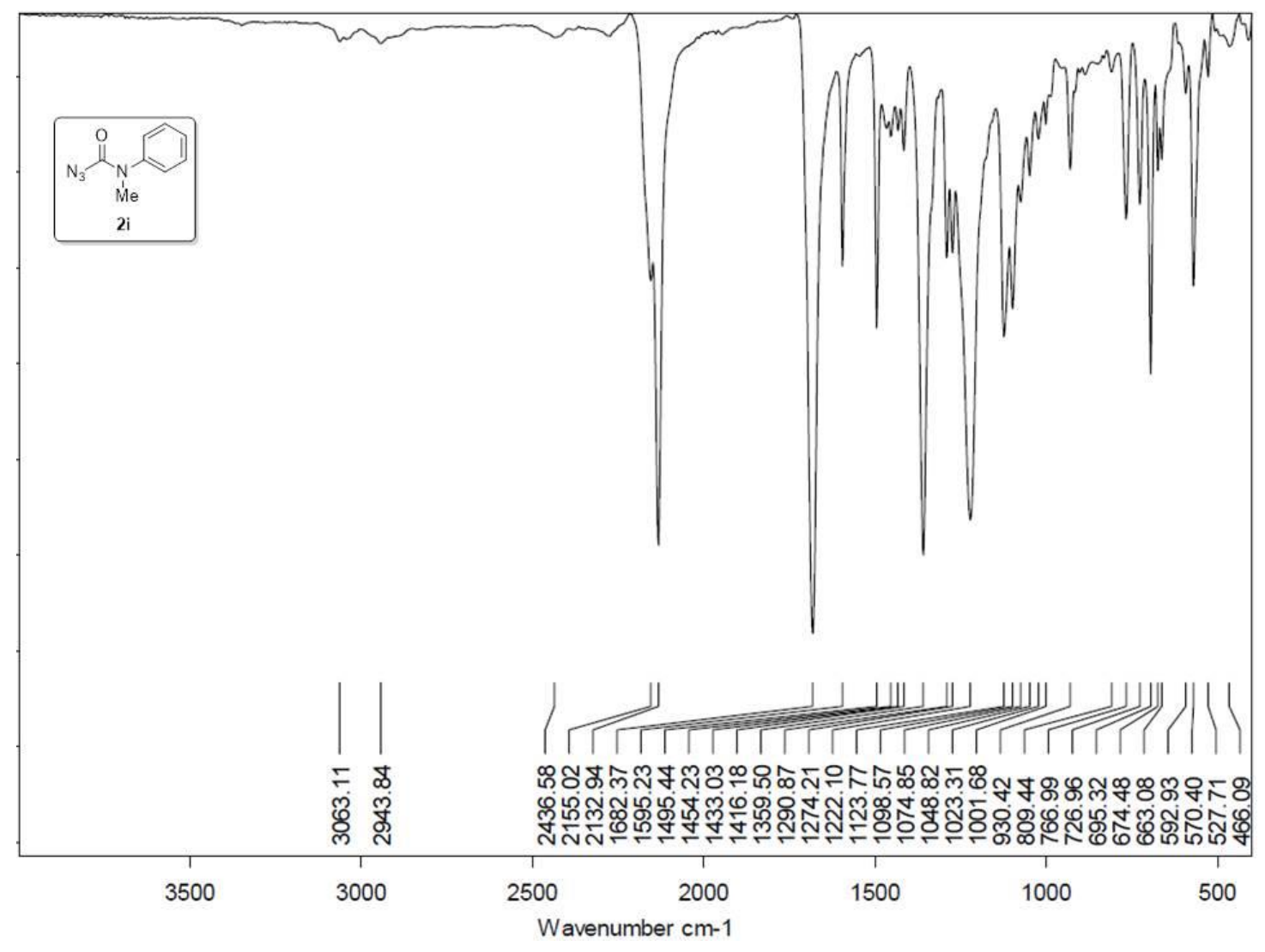


$\mathrm{N}$-(tert-Butyl)-2-(3-pentylureido)benzamide (Table 2, 3ab, ${ }^{1} \mathrm{H}$ NMR-500 MHz \& ${ }^{13} \mathrm{C}$ NMR-125 $\mathrm{MHz}$ in $\mathrm{CDCl}_{3}$ )
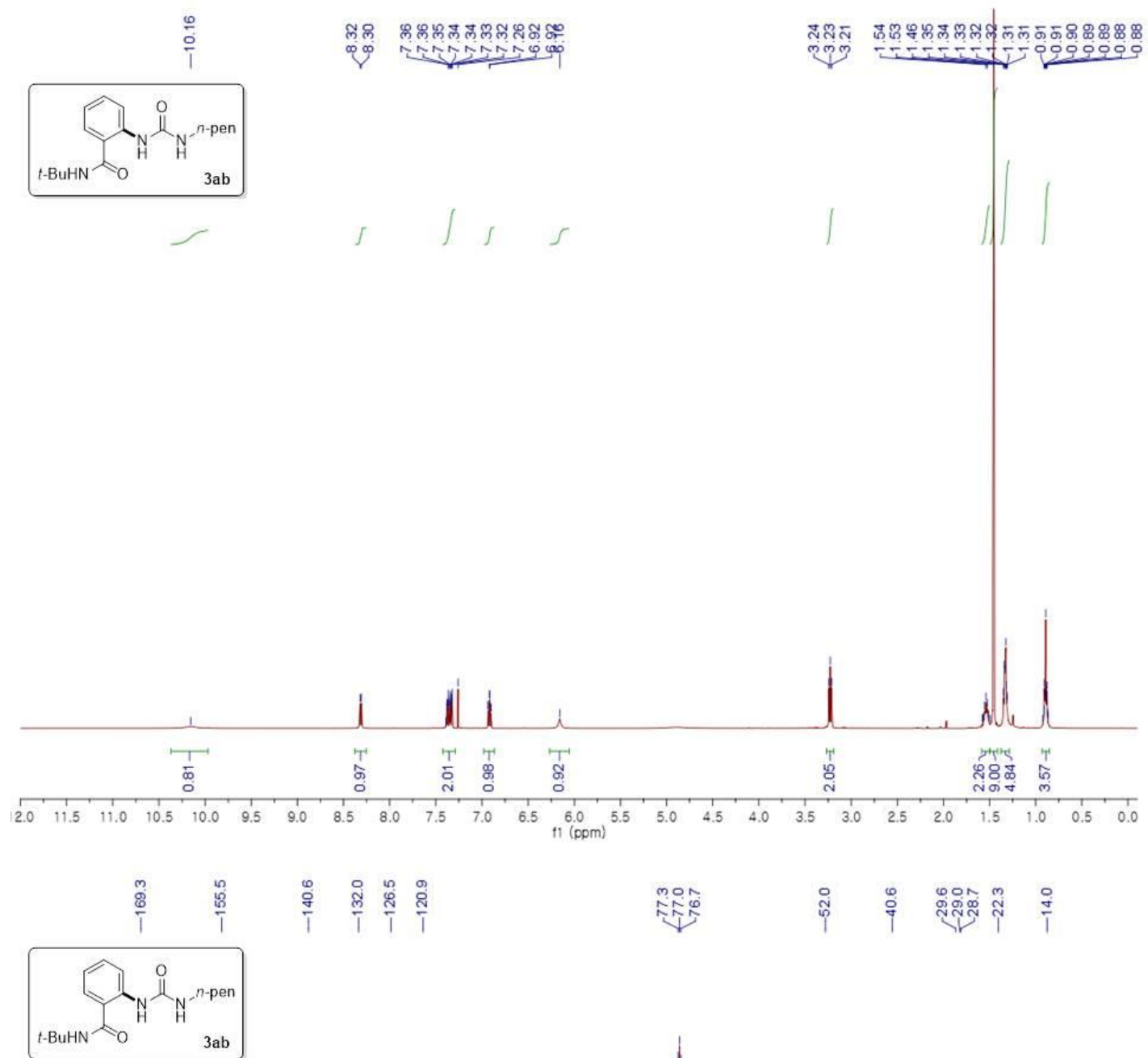

$3 a b$
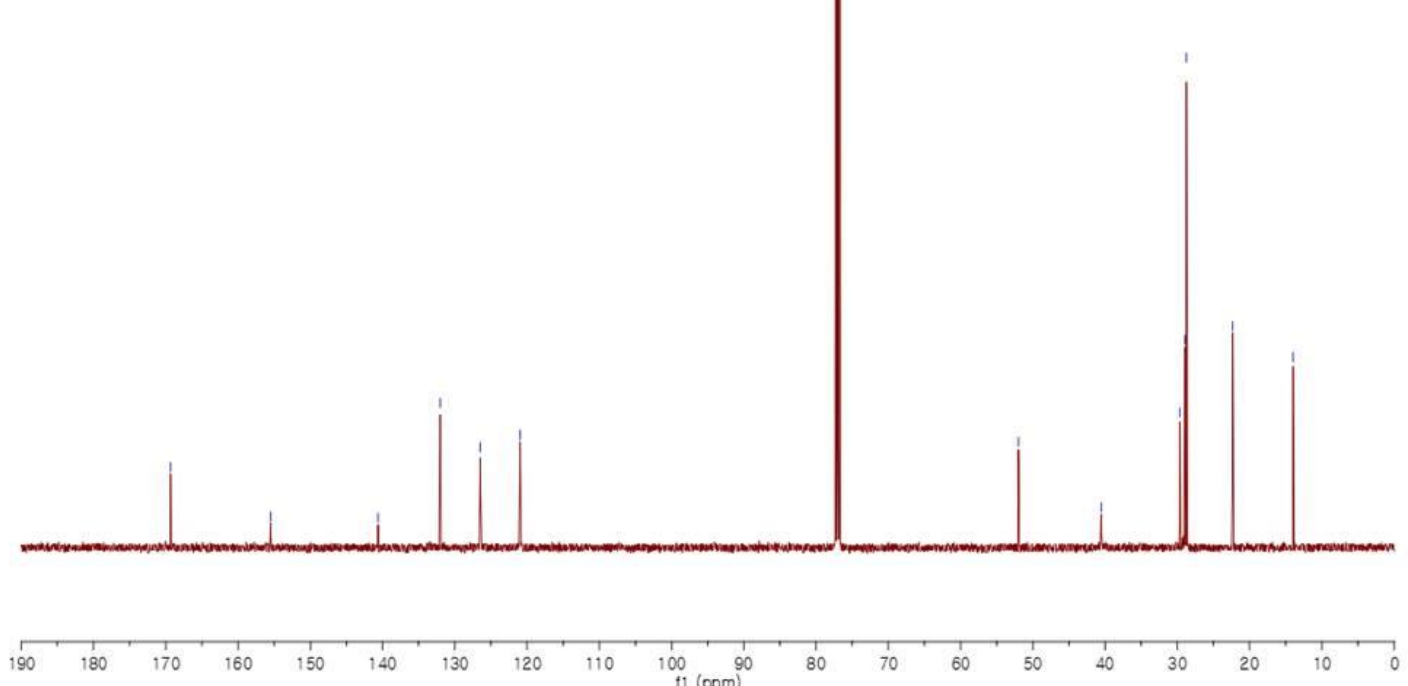


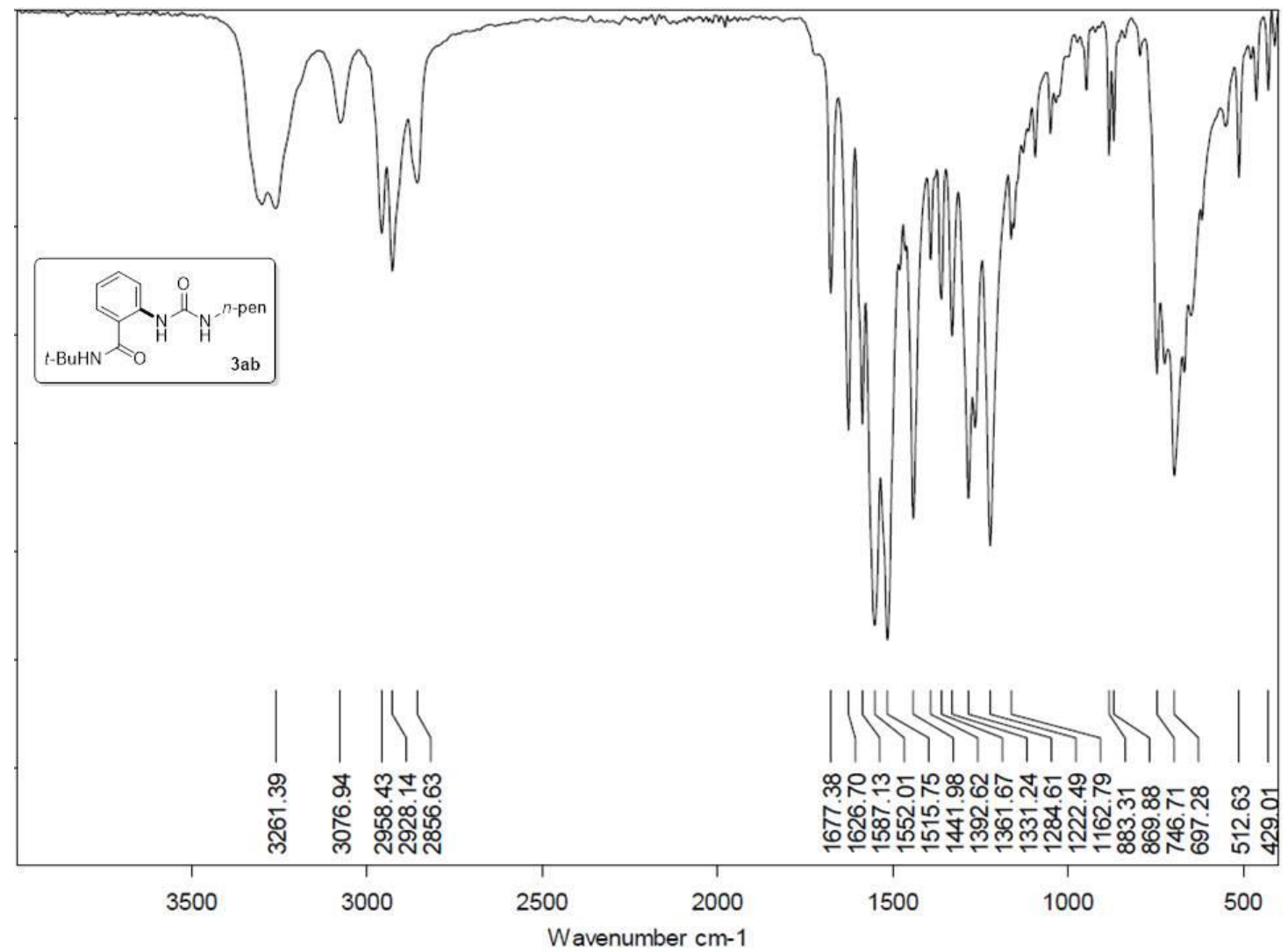


1,3-Dipentylurea (Scheme 2, 3ab', ${ }^{1} \mathrm{H}$ NMR-500 MHz \& ${ }^{13} \mathrm{C}$ NMR-125 MHz in $\mathrm{CDCl}_{3}$ )

$\stackrel{2}{i}$

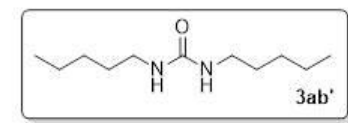

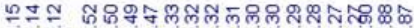

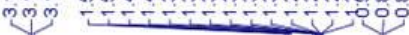
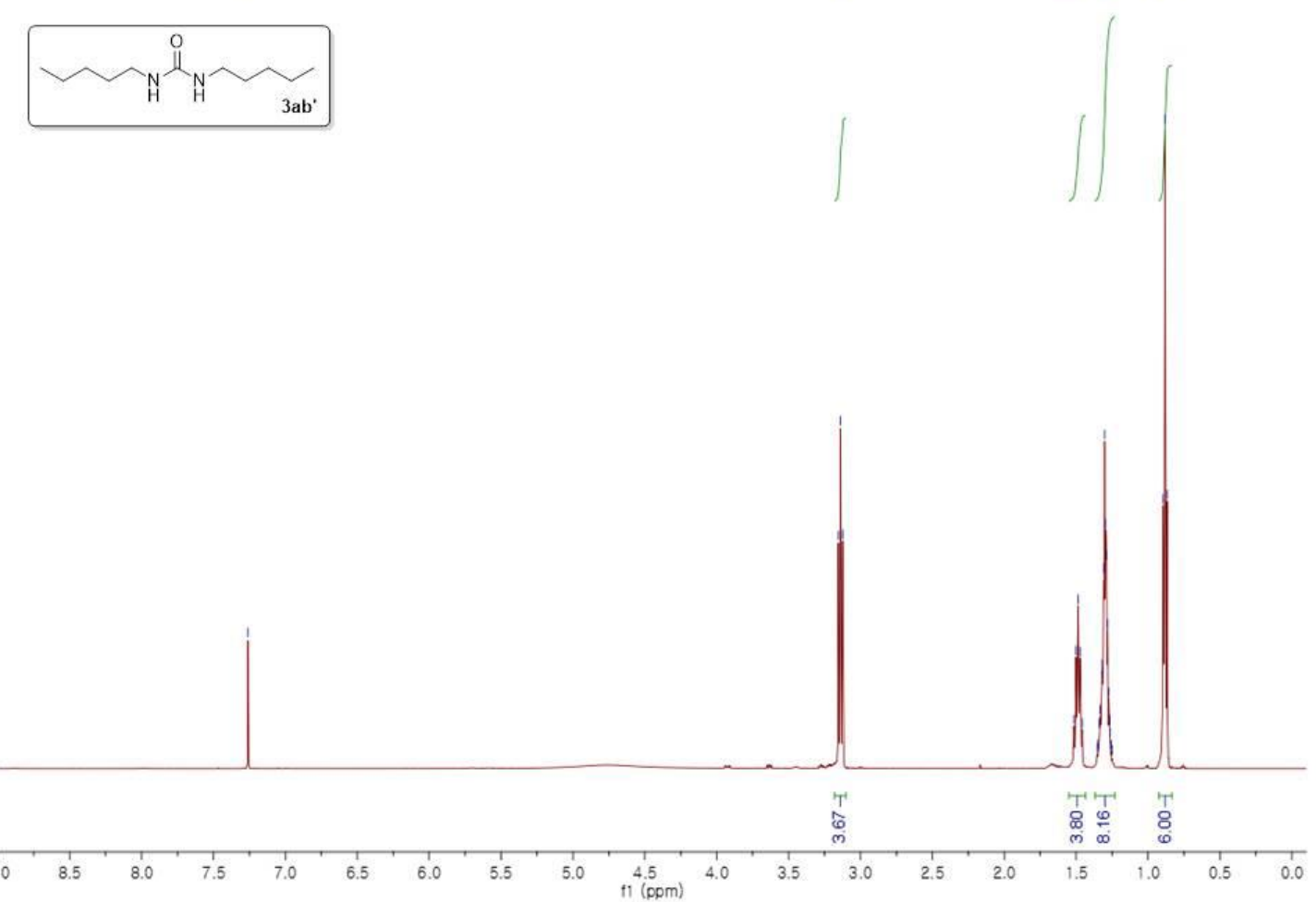

$\stackrel{0}{i}$

ำ

芦

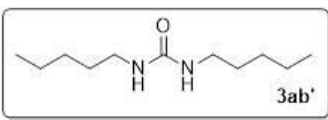

$3 \mathrm{ab}^{\circ}$

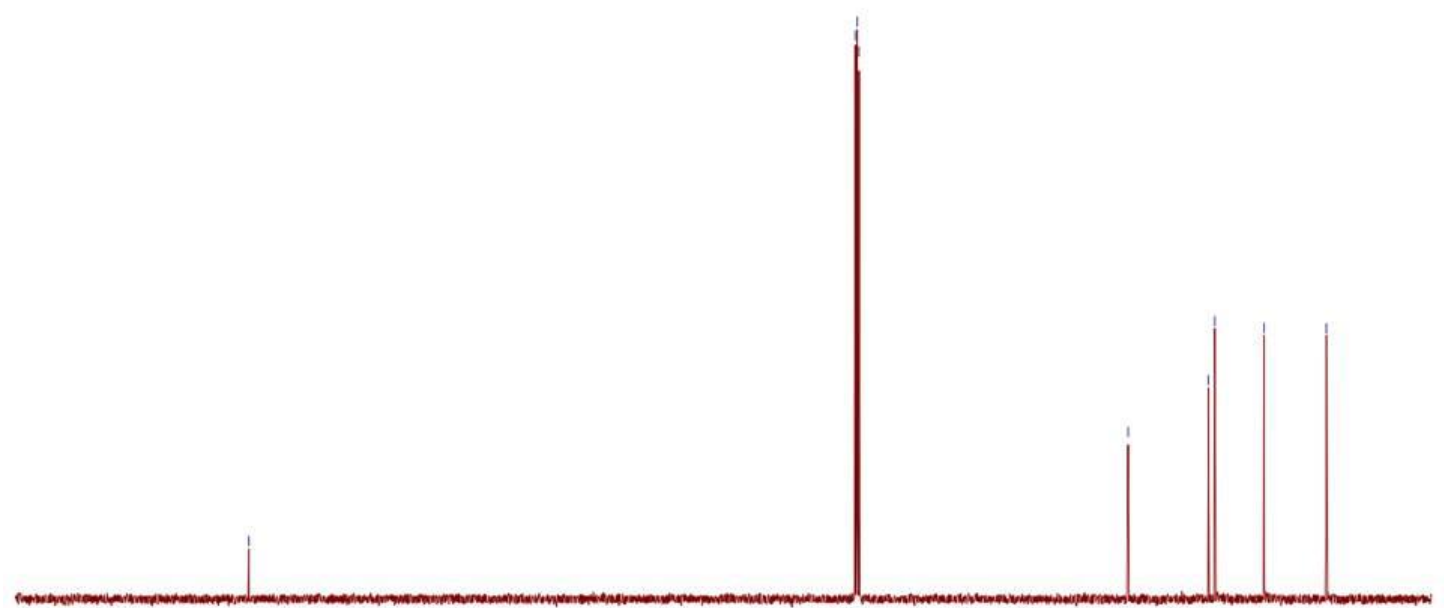

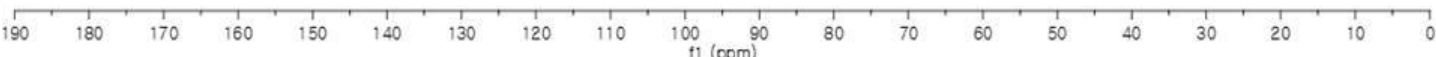




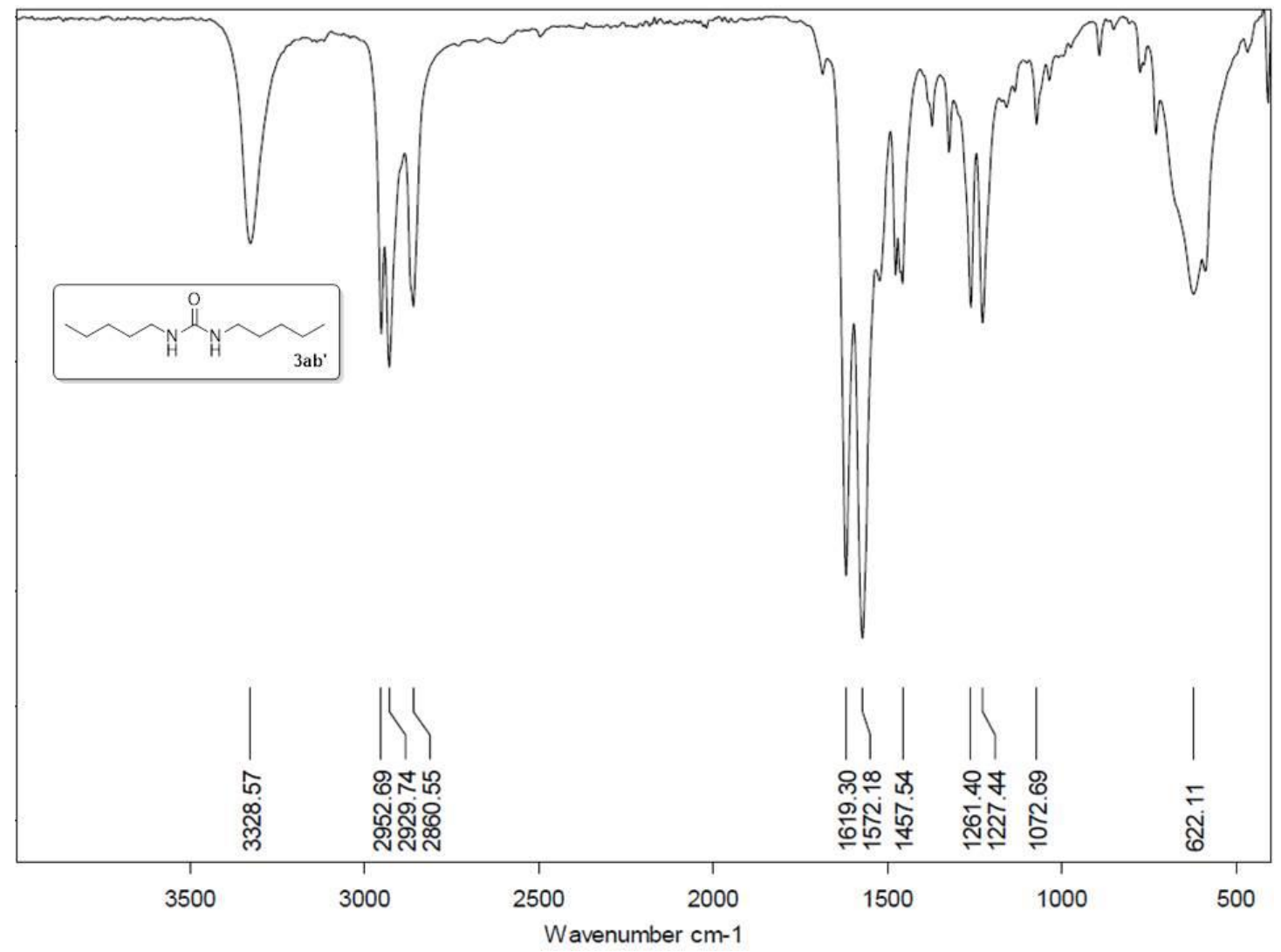


$N$-(tert-Butyl)-2-(3,3-diphenylureido)benzamide (Table 2, 3ac, ${ }^{1} \mathrm{H}$ NMR-500 MHz \& ${ }^{13} \mathrm{C}$ NMR-125 $\mathrm{MHz}$ in Acetone- $\left.d_{6}\right)$

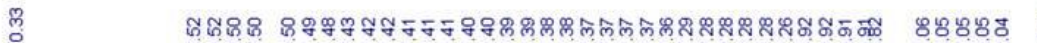
I

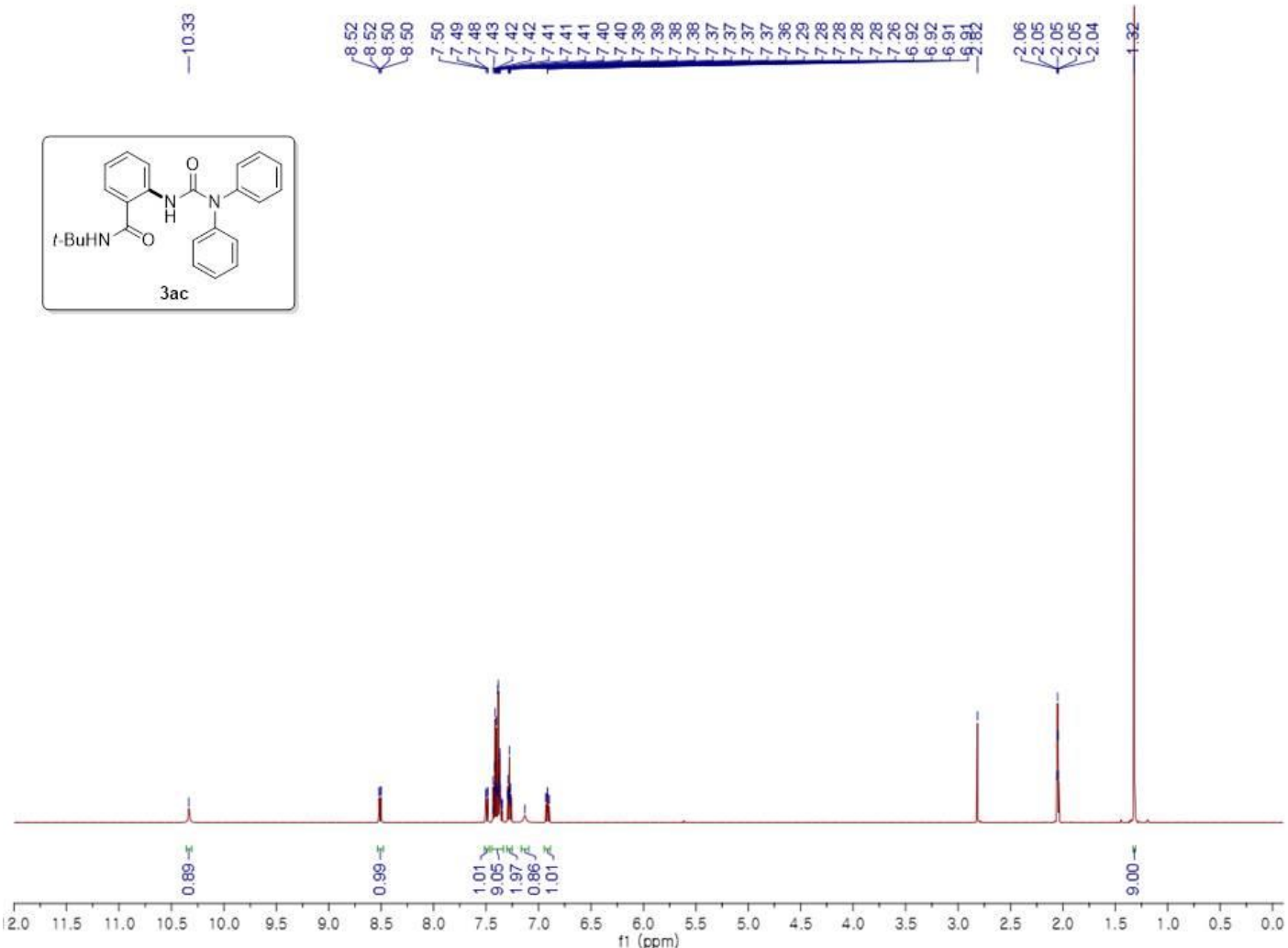

कृ

至

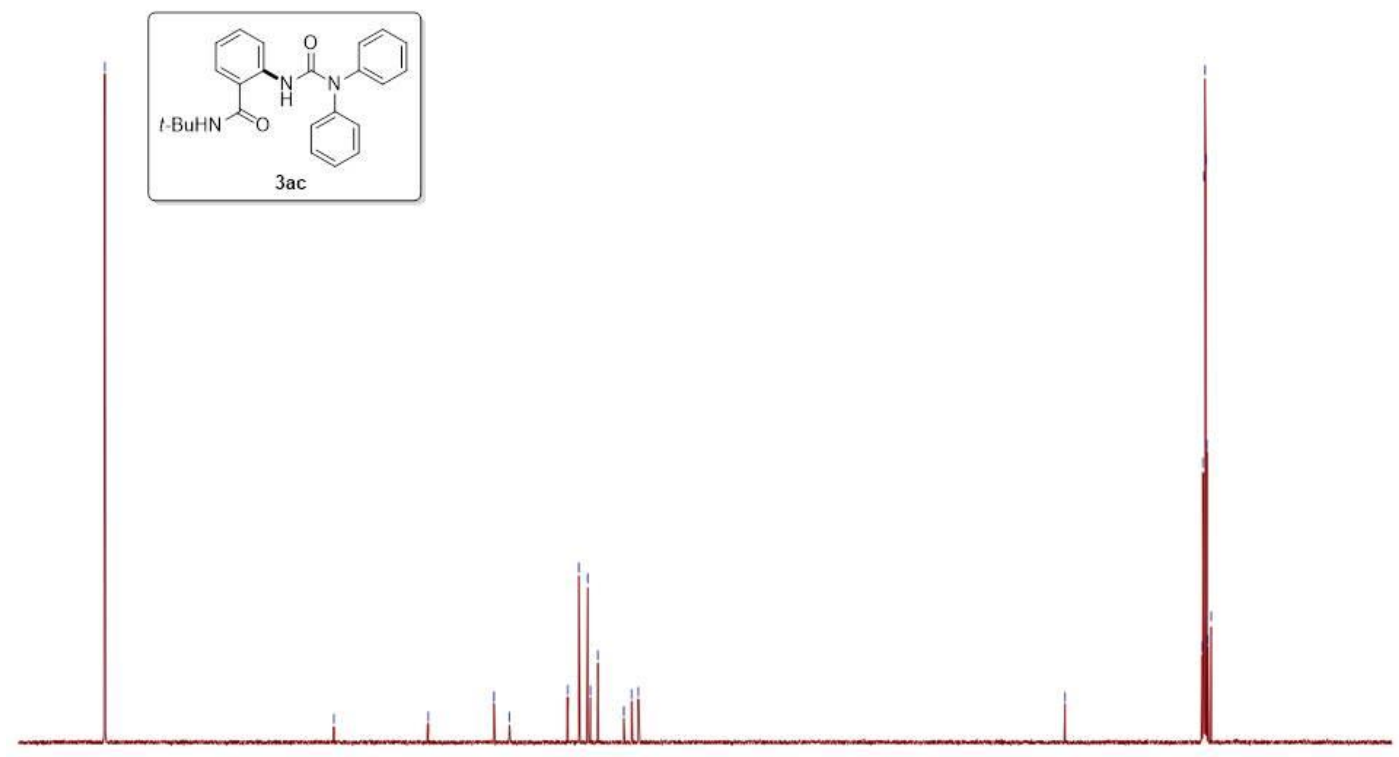

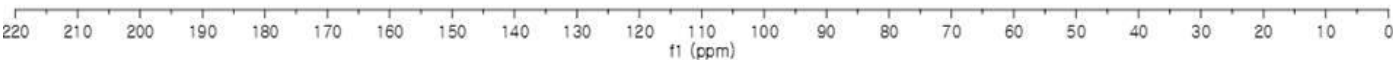




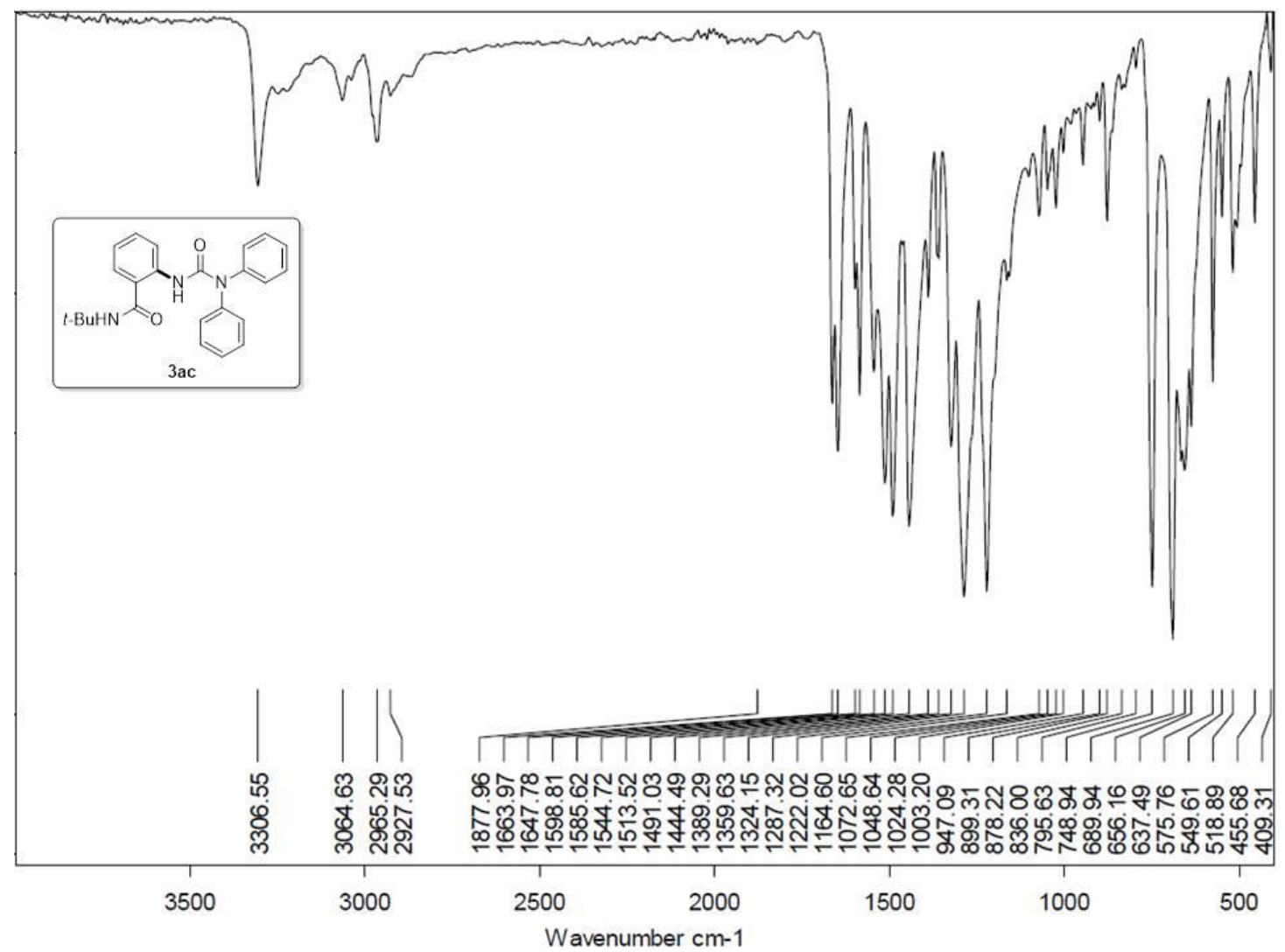


$\boldsymbol{N}$-(tert-Butyl)-2-(3,3-di-p-tolylureido)benzamide (Table 2, 3ad, ${ }^{1} \mathrm{H}$ NMR-500 MHz \& ${ }^{13} \mathrm{C}$ NMR$125 \mathrm{MHz}$ in Acetone- $\left.d_{6}\right)$
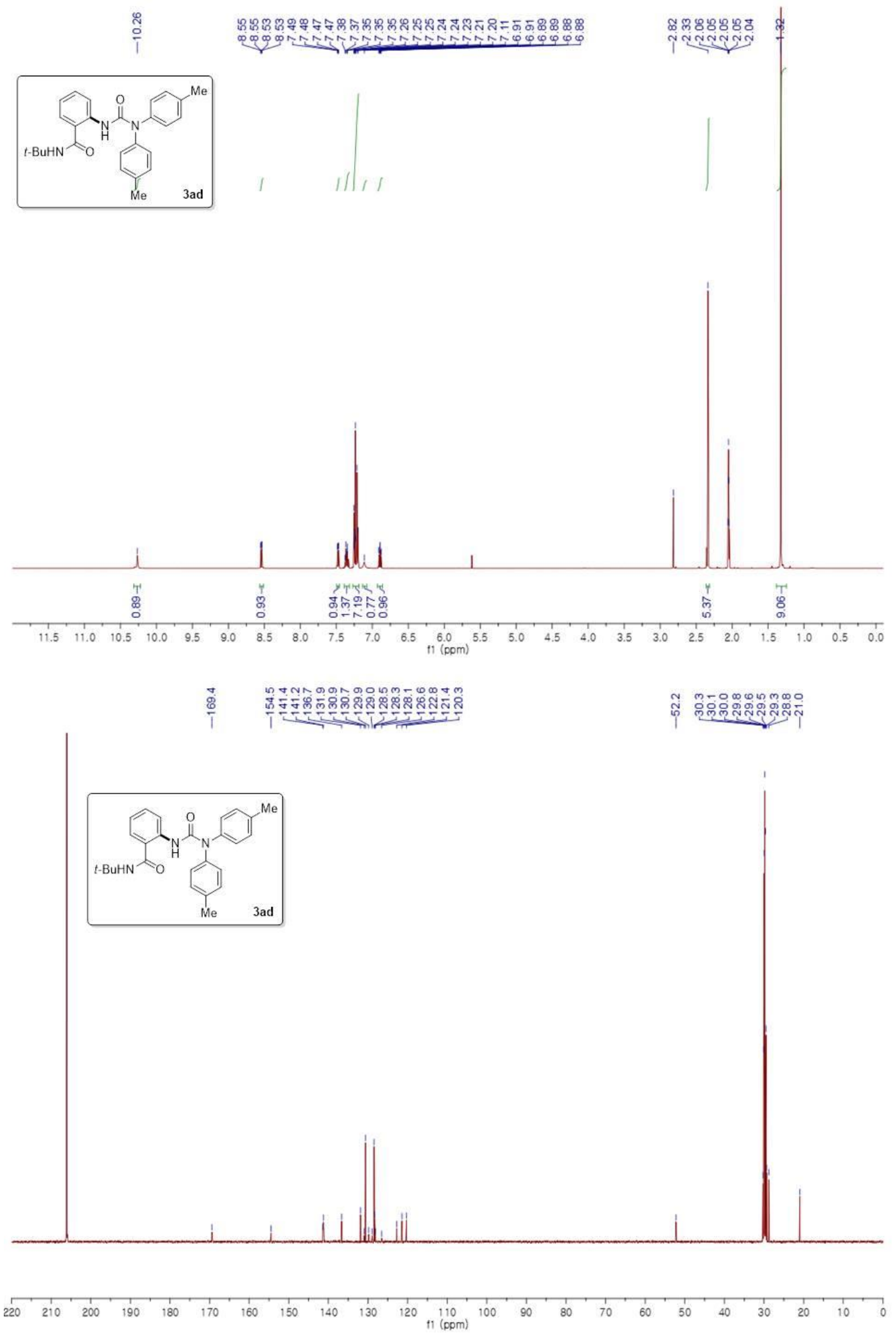


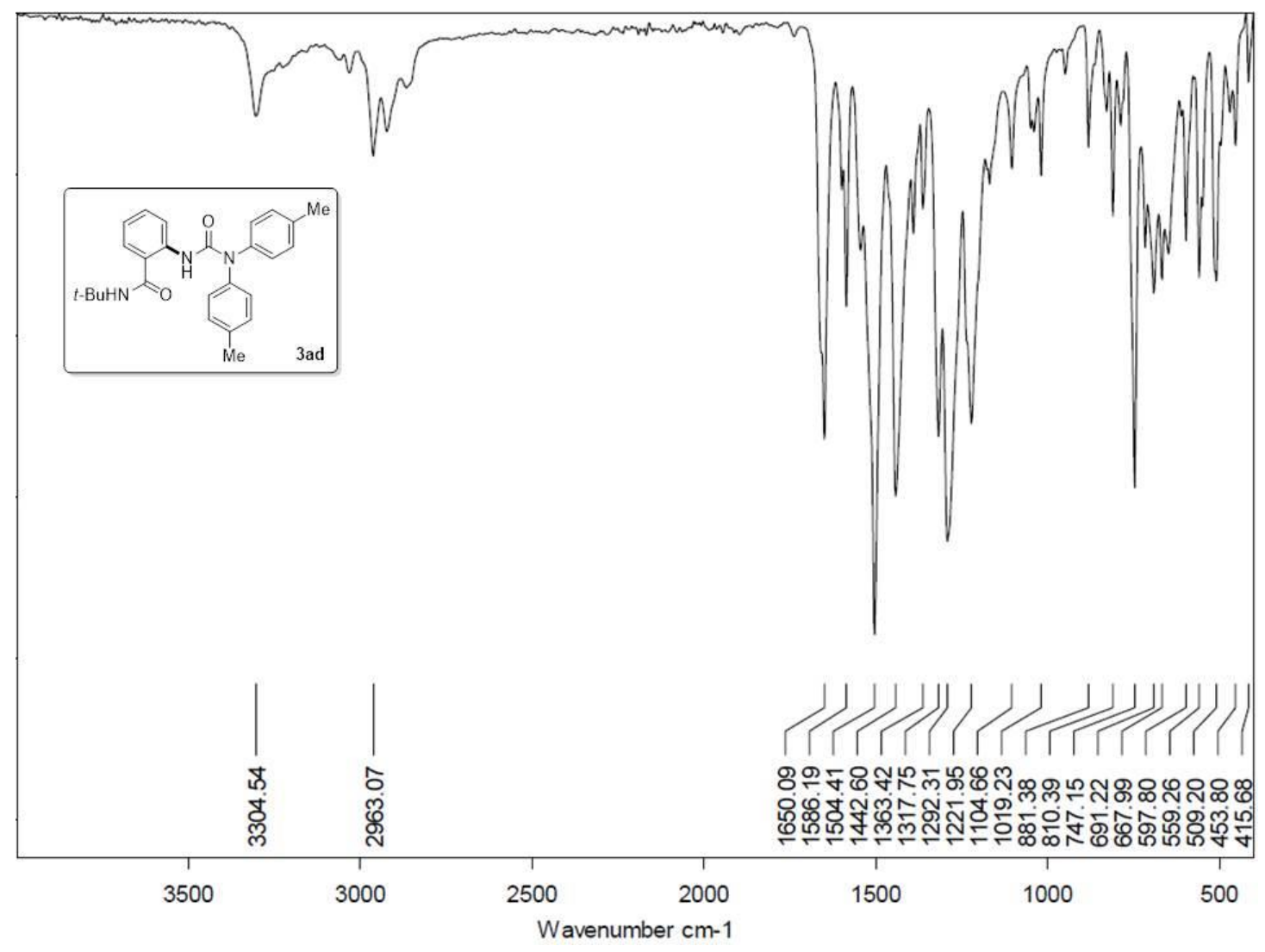


$N$-(tert-Butyl)-2-(3-(4-(tert-butyl)phenyl)-3-(p-tolyl)ureido)benzamide (Table 2, 3ae, ${ }^{1} \mathrm{H}$ NMR-500 $\mathrm{MHz} \&{ }^{13} \mathrm{C}$ NMR-125 MHz in Acetone- $\left.d_{6}\right)$

లొ

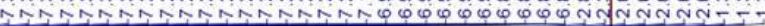
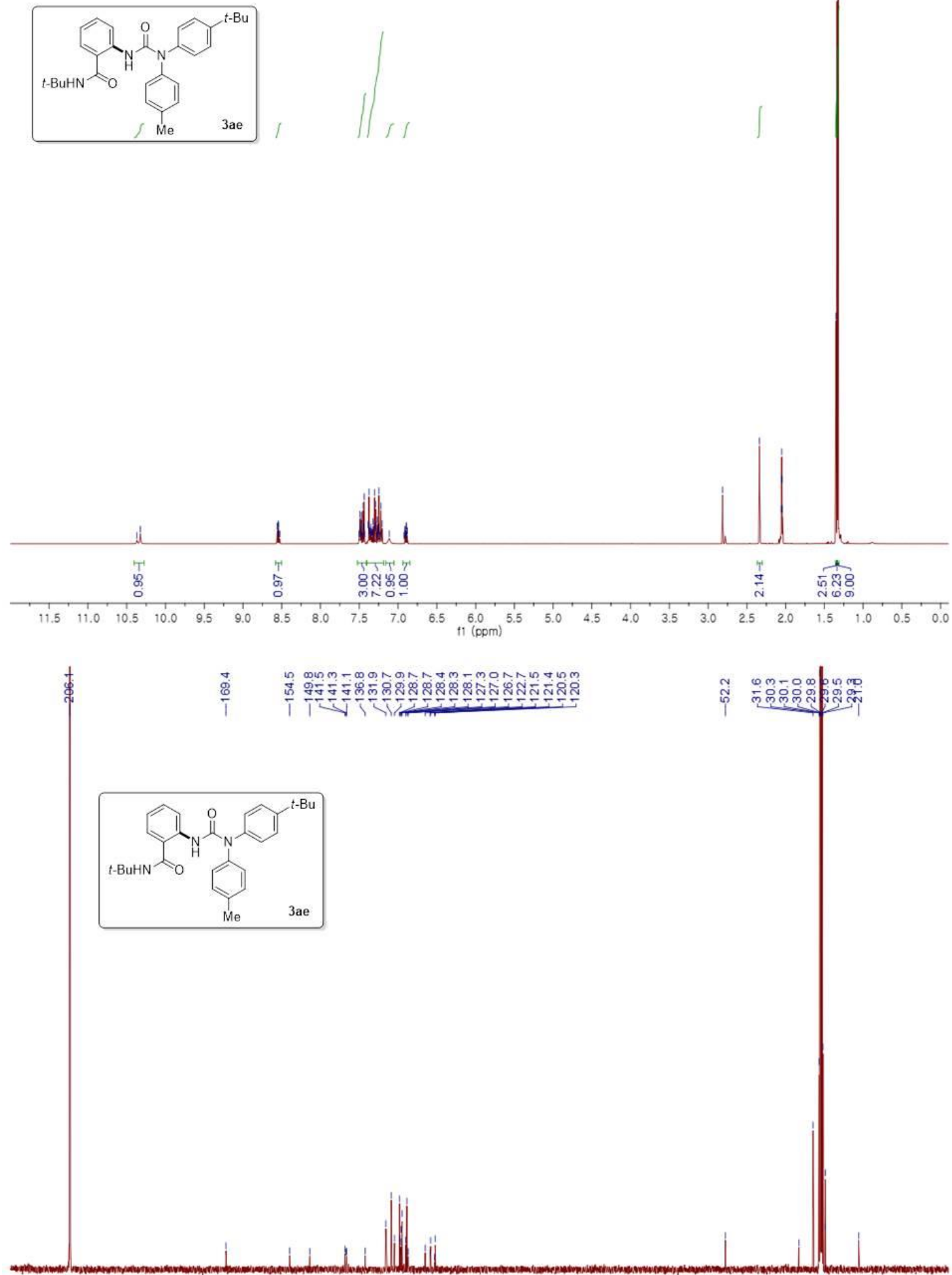

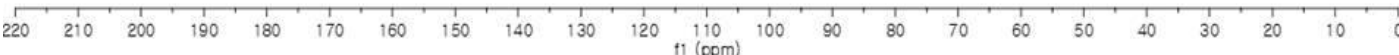




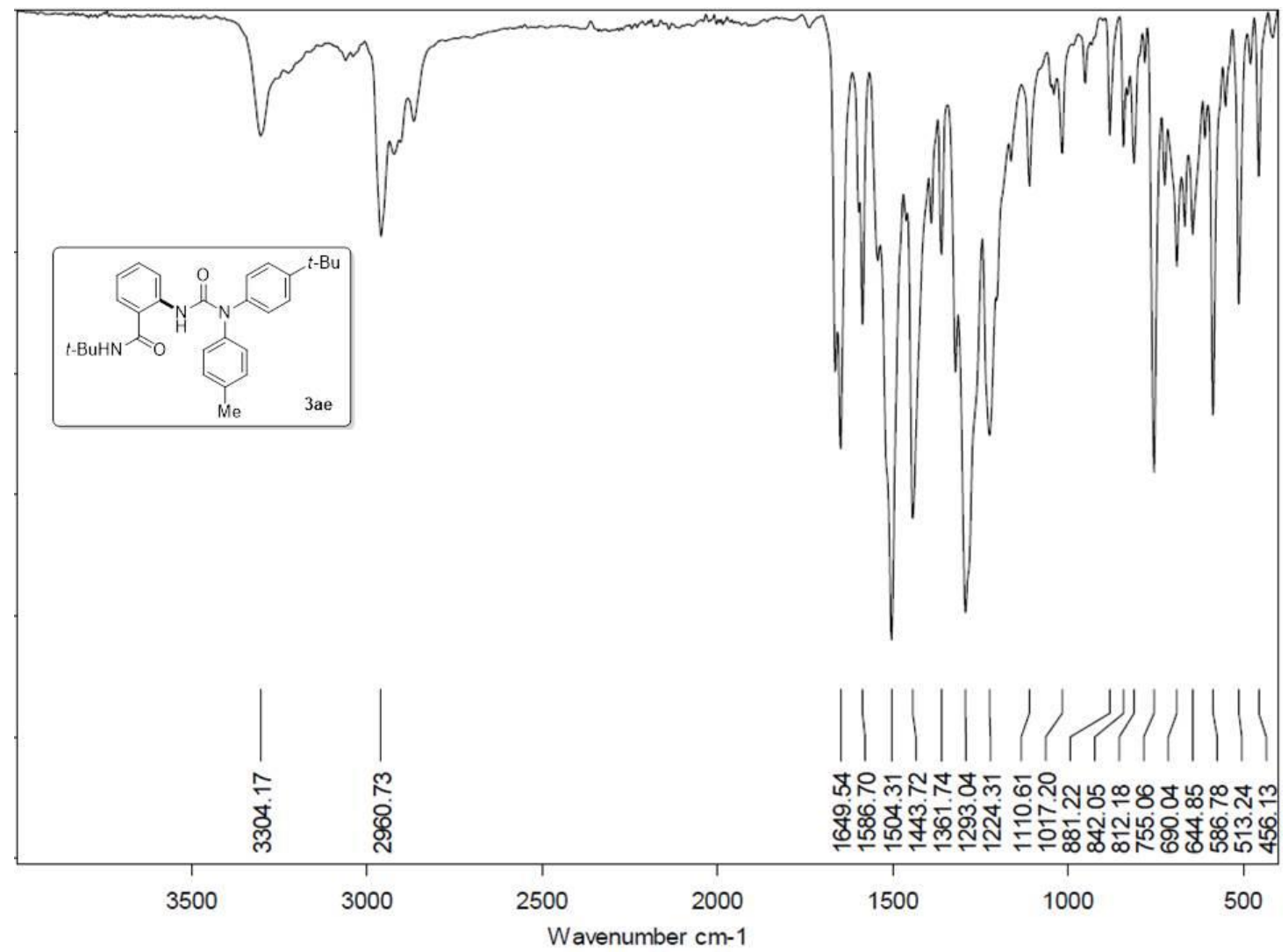


$\mathrm{N}$-(tert-Butyl)-2-(3-(4-chlorophenyl)-3-(4-fluorophenyl)ureido)benzamide (Table 2, 3af, ${ }^{1} \mathrm{H}$ NMR$500 \mathrm{MHz} \&{ }^{13} \mathrm{C}$ NMR-125 MHz in Acetone- $\left.d_{6}\right)$

$\stackrel{8}{0}$

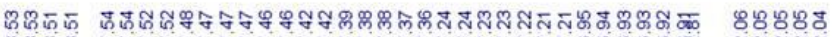

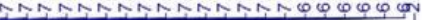

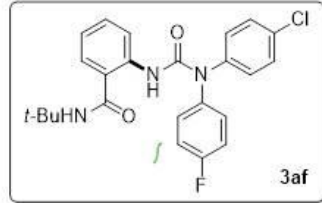

年
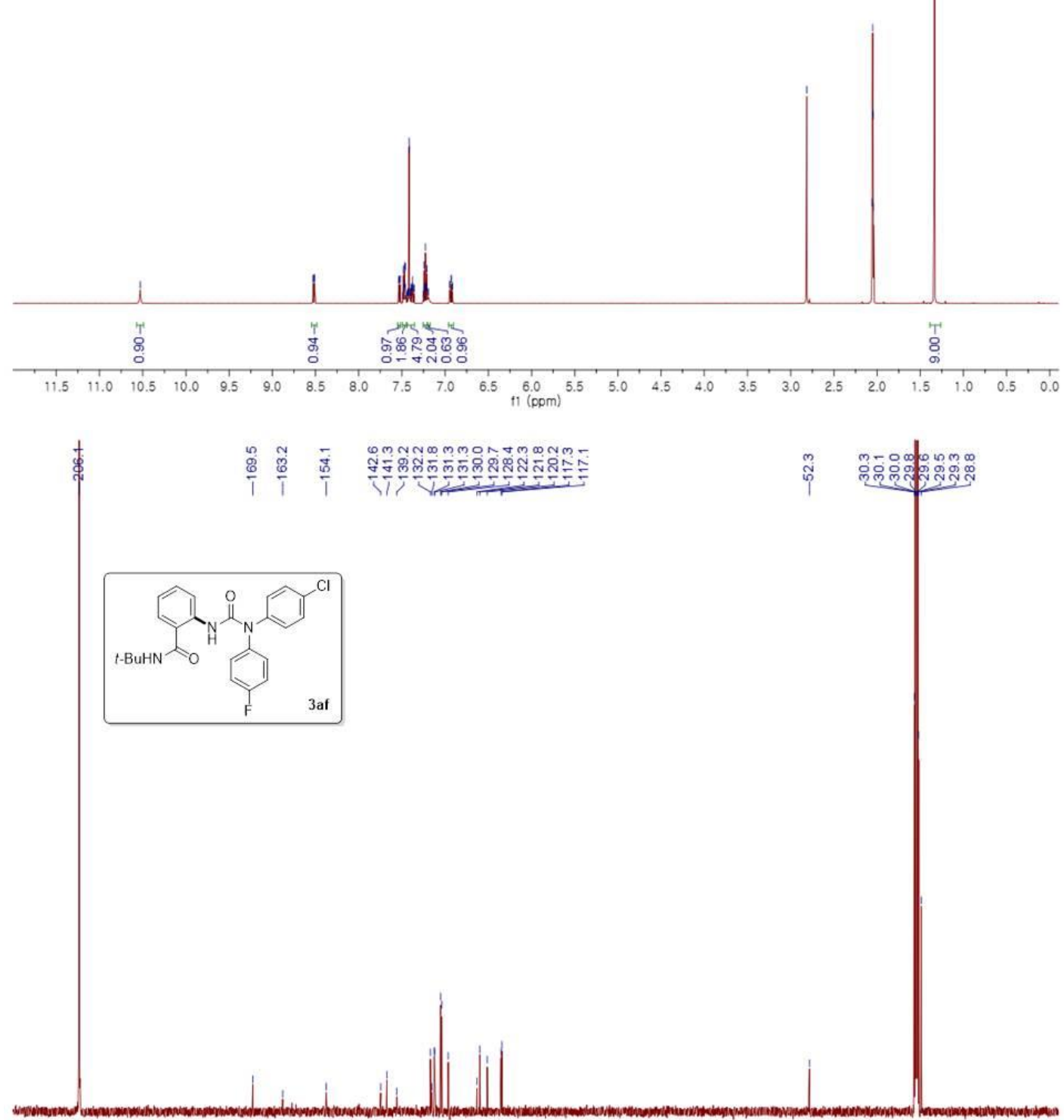

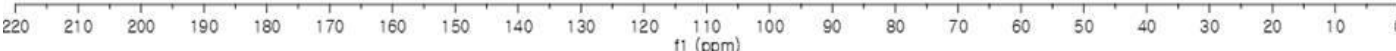




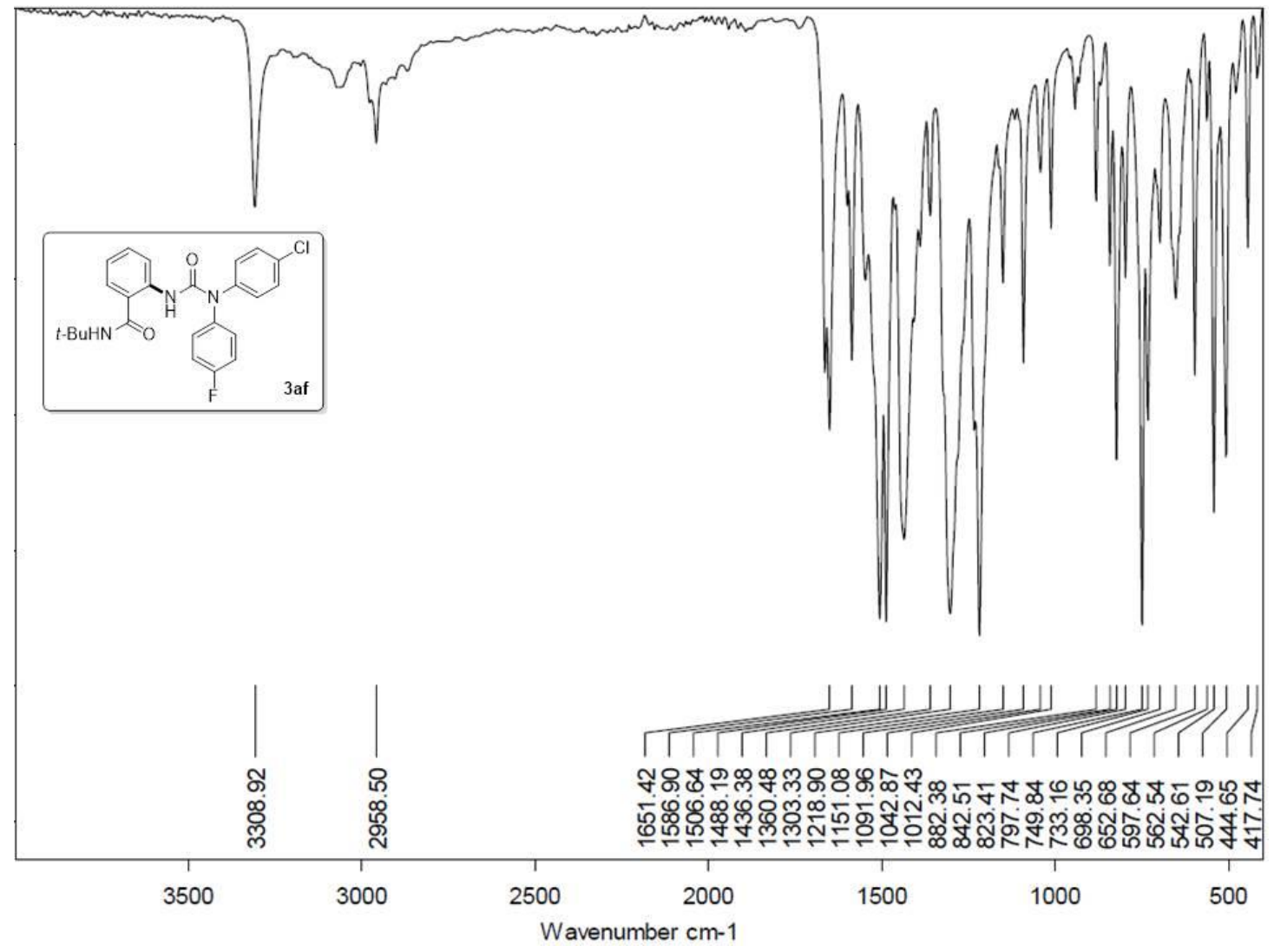


$\mathrm{N}$-(tert-Butyl)-2-(3-(4-fluoro-2-methylphenyl)-3-(4-fluorophenyl)ureido)benzamide (Table 2, 3ag, ${ }^{1} \mathrm{H}$ NMR-500 MHz \& ${ }^{13} \mathrm{C}$ NMR-125 MHz in Acetone- $\left.d_{6}\right)$
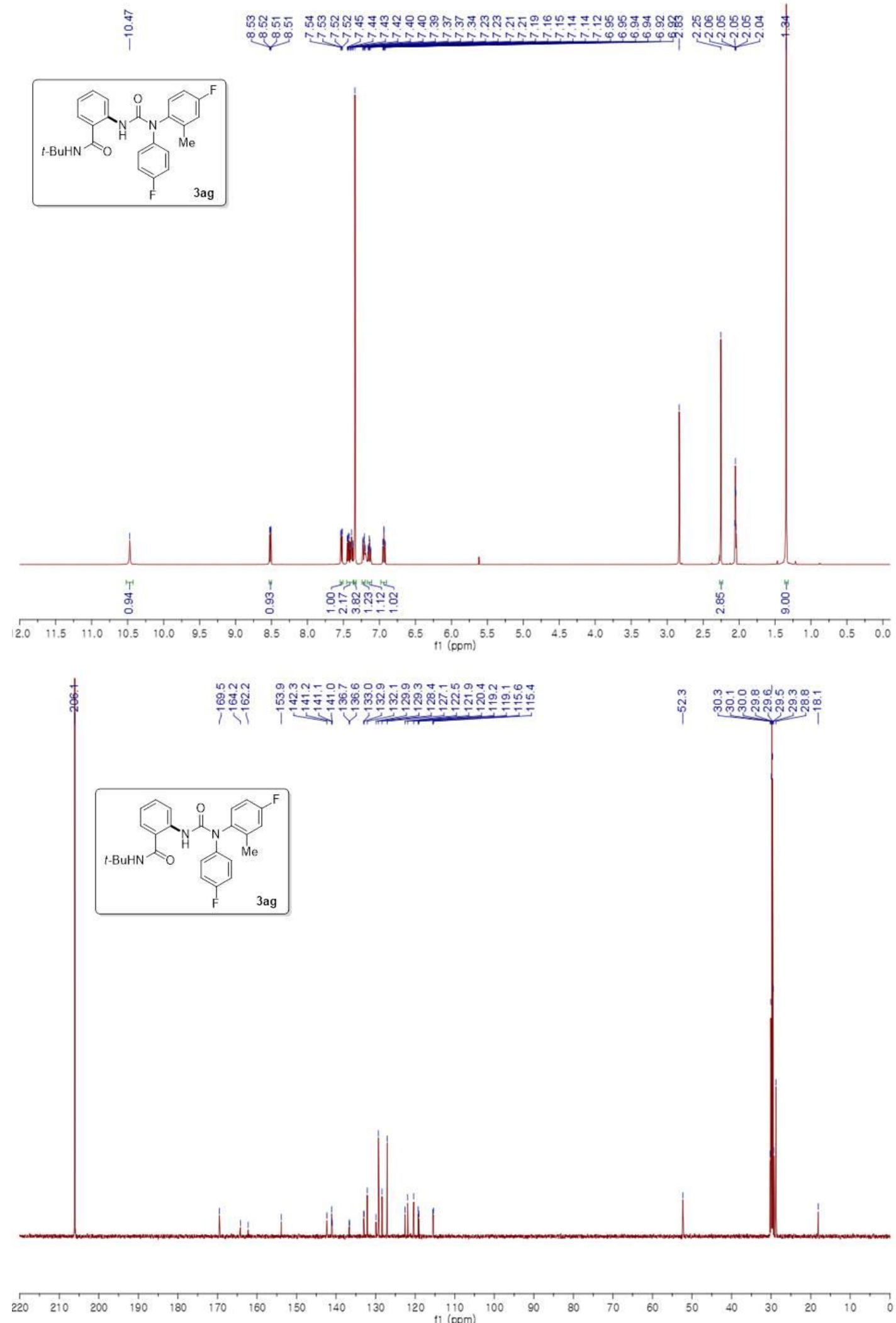


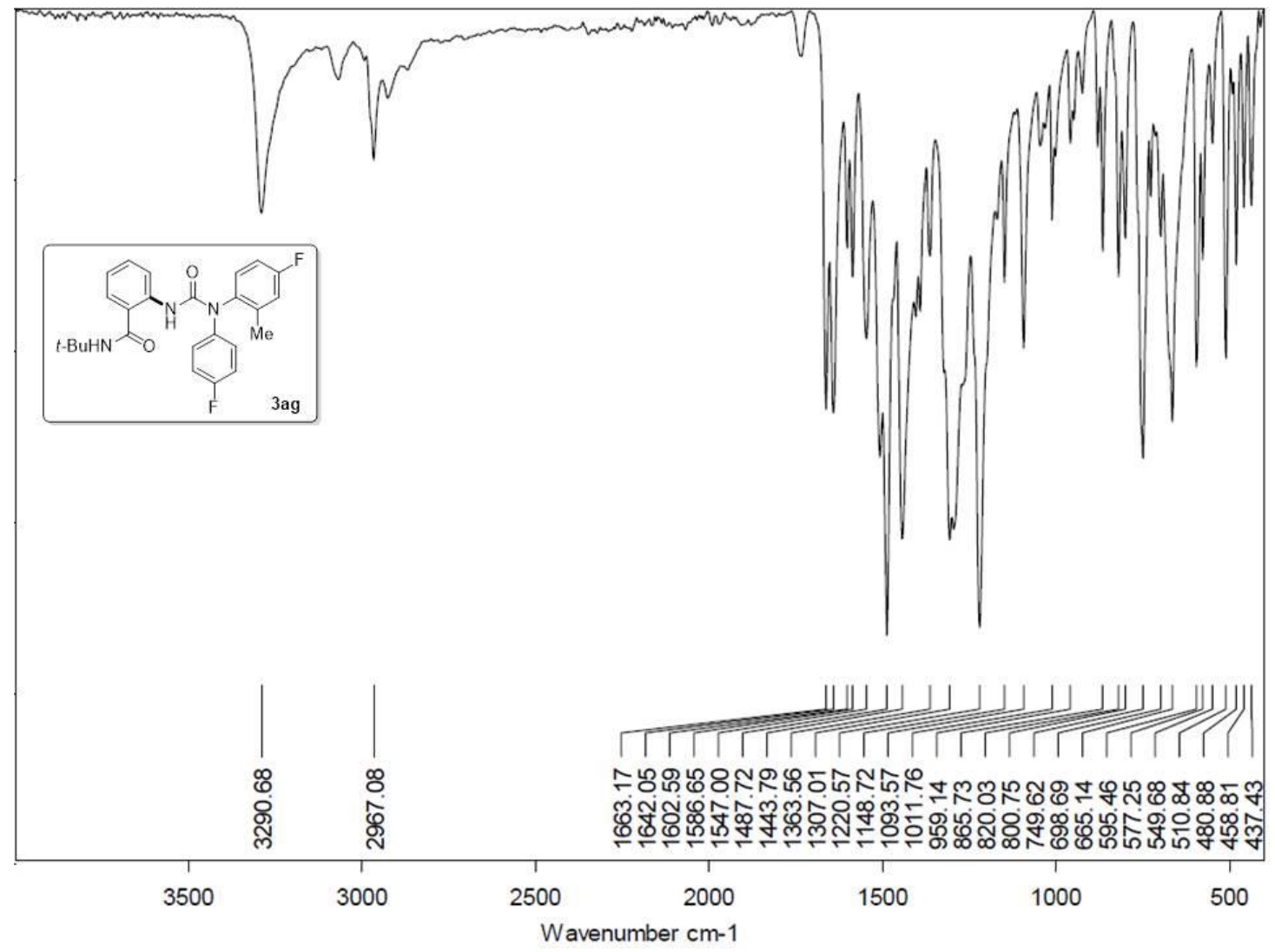


2-(3-Benzyl-3-phenylureido)- $N$-(tert-butyl)benzamide (Table 2, 3ah, ${ }^{1} \mathrm{H}$ NMR-500 MHz \& ${ }^{13} \mathrm{C}$ NMR-125 MHz in Acetone- $d_{6}$ )

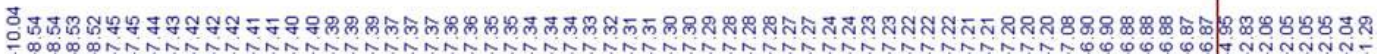
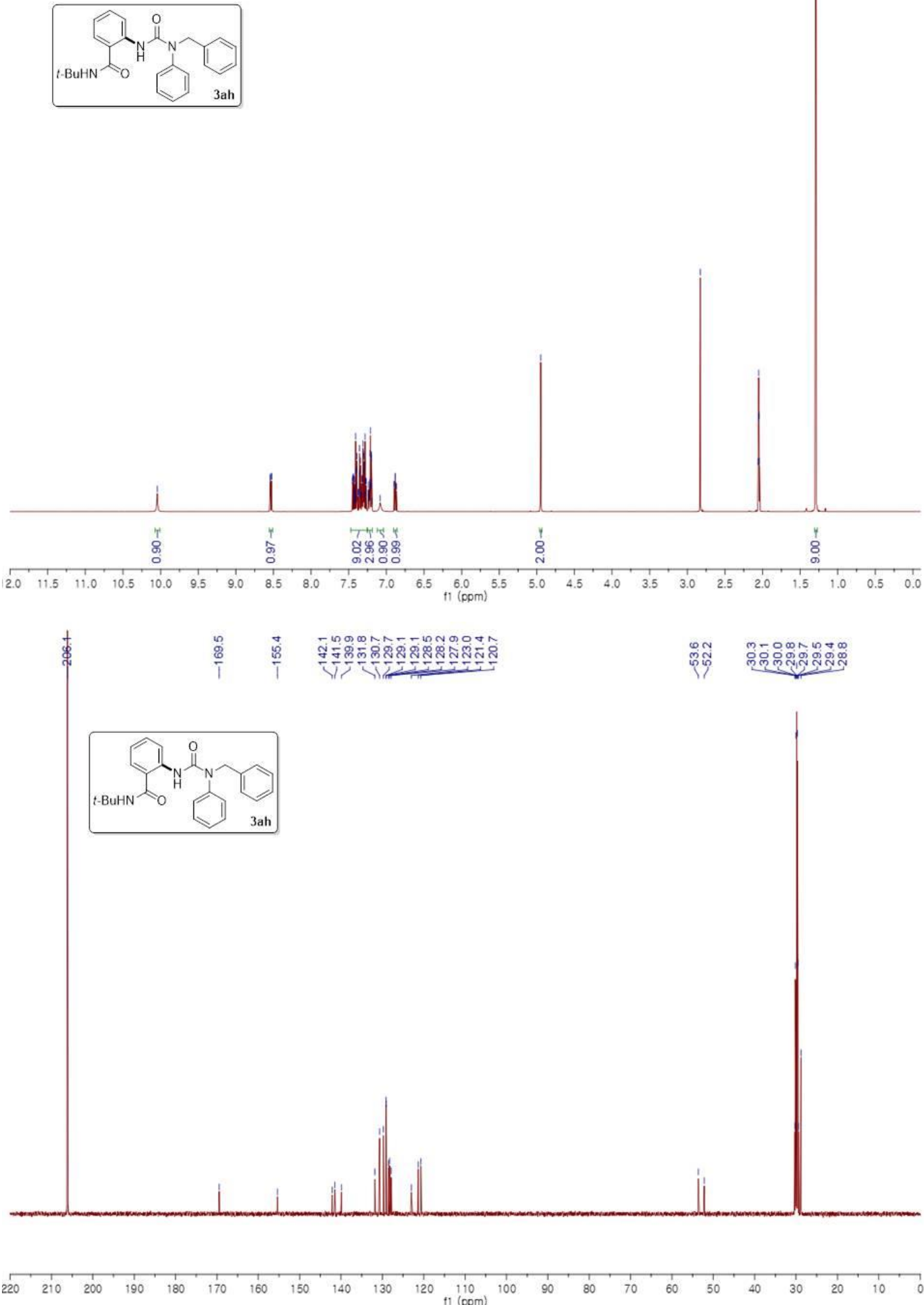


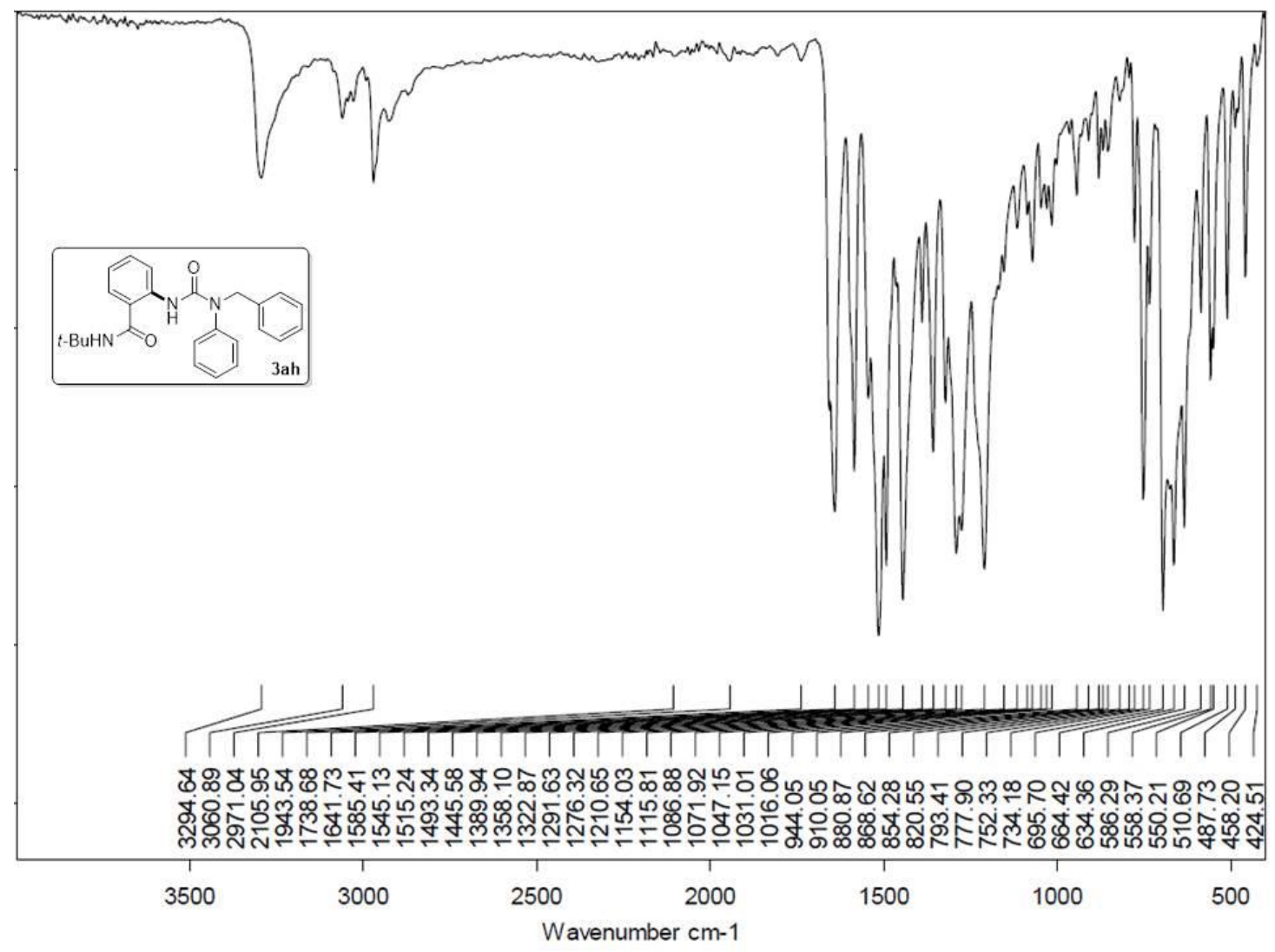


$\boldsymbol{N}$-(tert-Butyl)-2-(3-methyl-3-phenylureido)benzamide (Table 2, 3ai, ${ }^{1} \mathrm{H}$ NMR-500 MHz \& ${ }^{13} \mathrm{C}$ NMR-125 MHz in Acetone- $d_{6}$ )
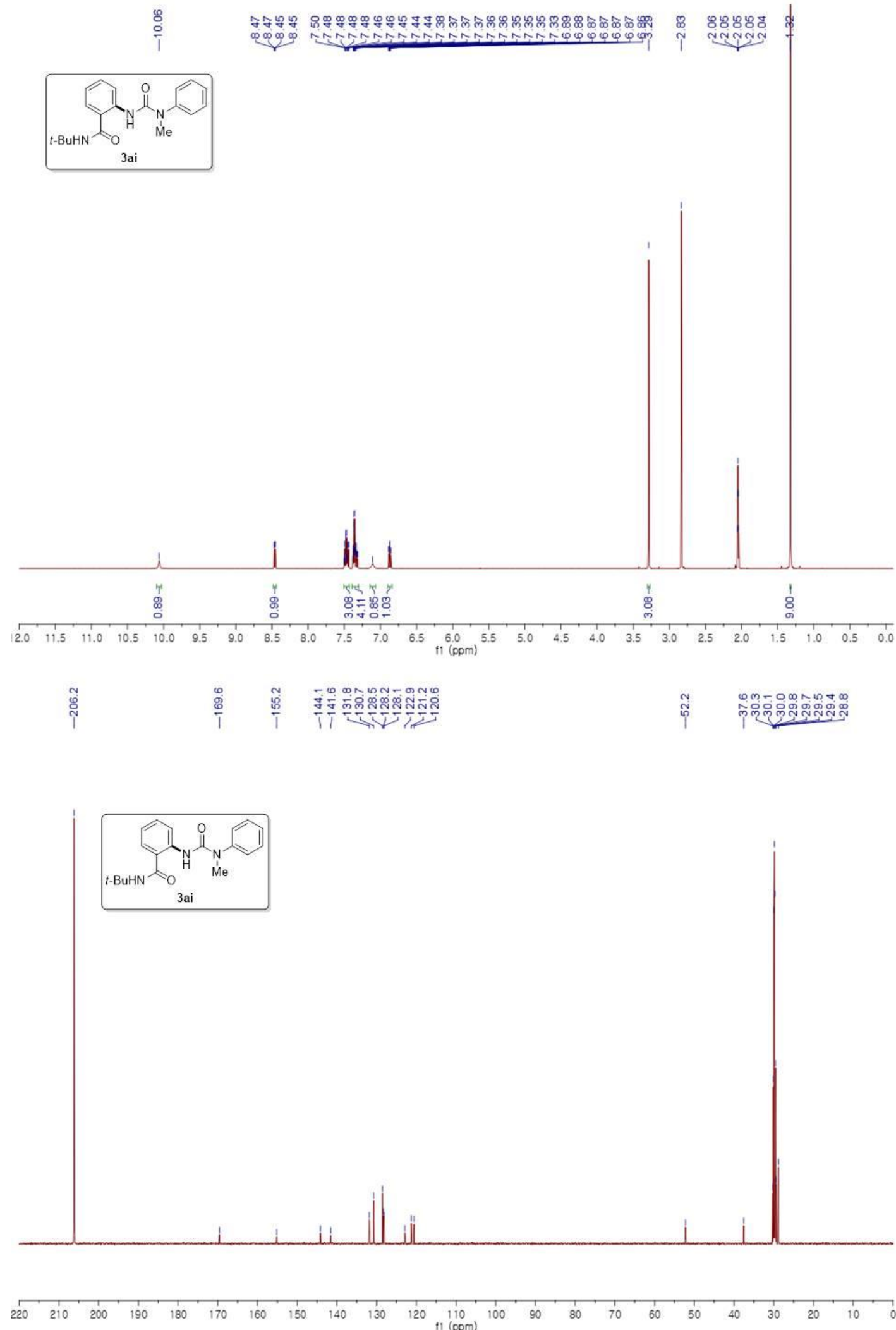


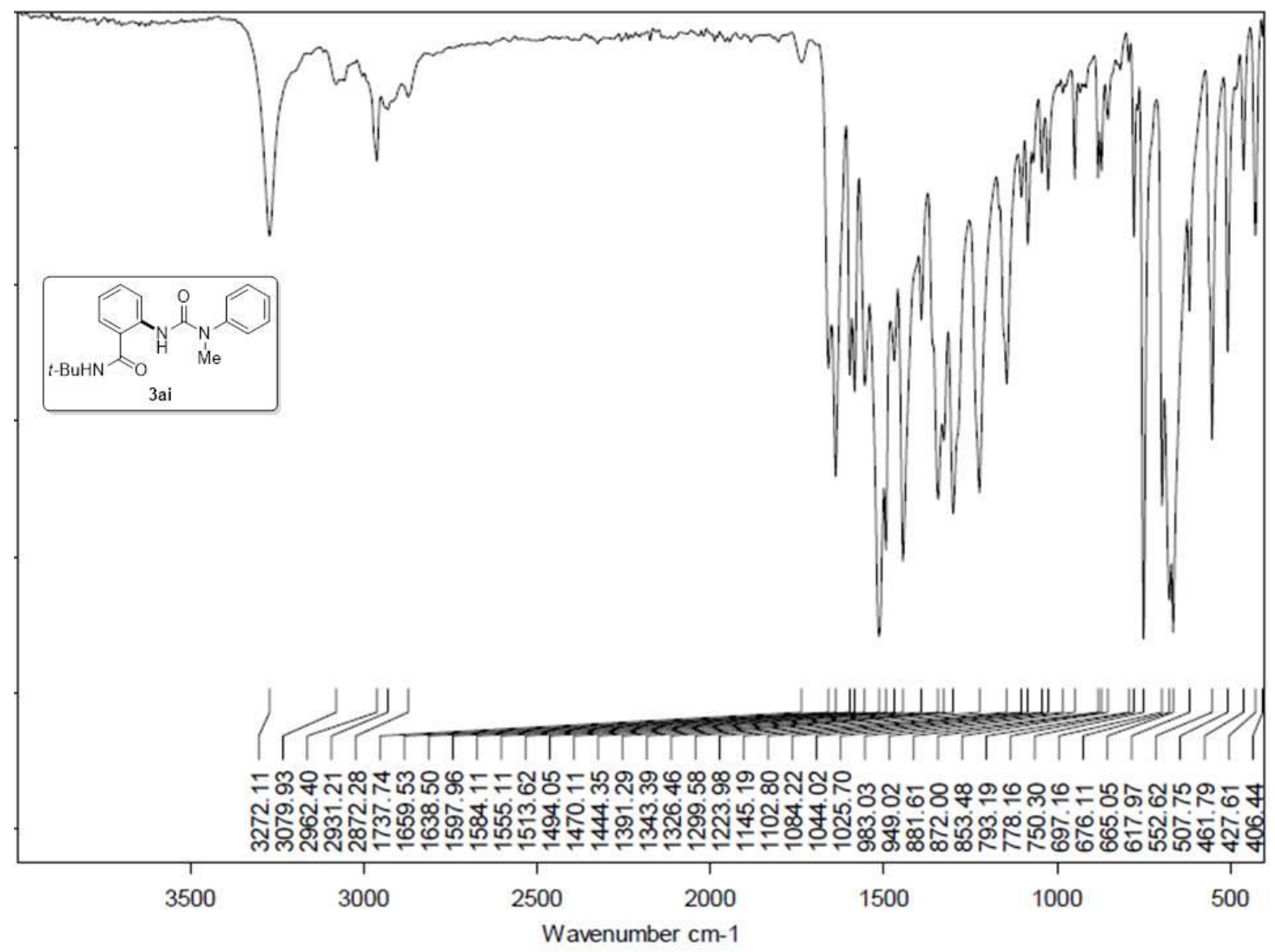


$\mathrm{N}$-(2-(tert-Butylcarbamoyl)phenyl)pyrrolidine-1-carboxamide (Table 2, 3aj, ${ }^{1} \mathrm{H}$ NMR-500 MHz \& ${ }^{13} \mathrm{C}$ NMR-125 $\mathrm{MHz}$ in $\mathrm{CDCl}_{3}$ )

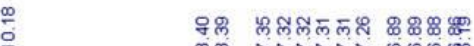

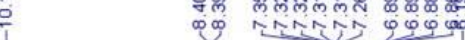

$t$-BuHN

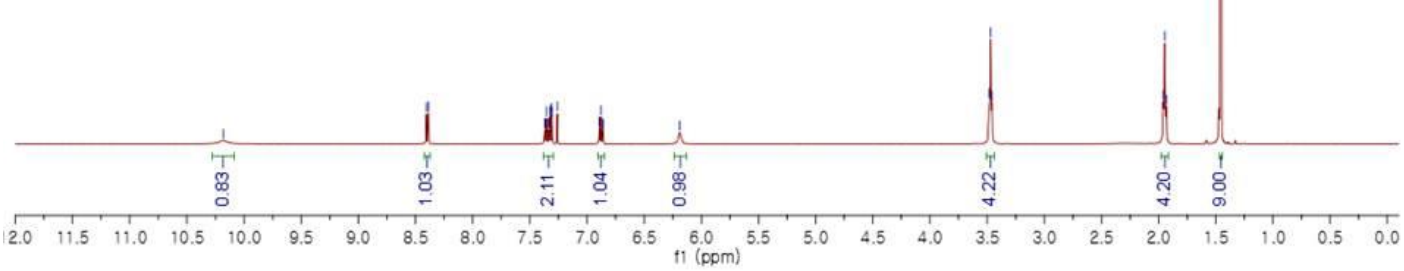

广

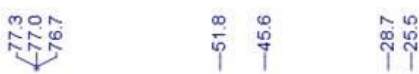
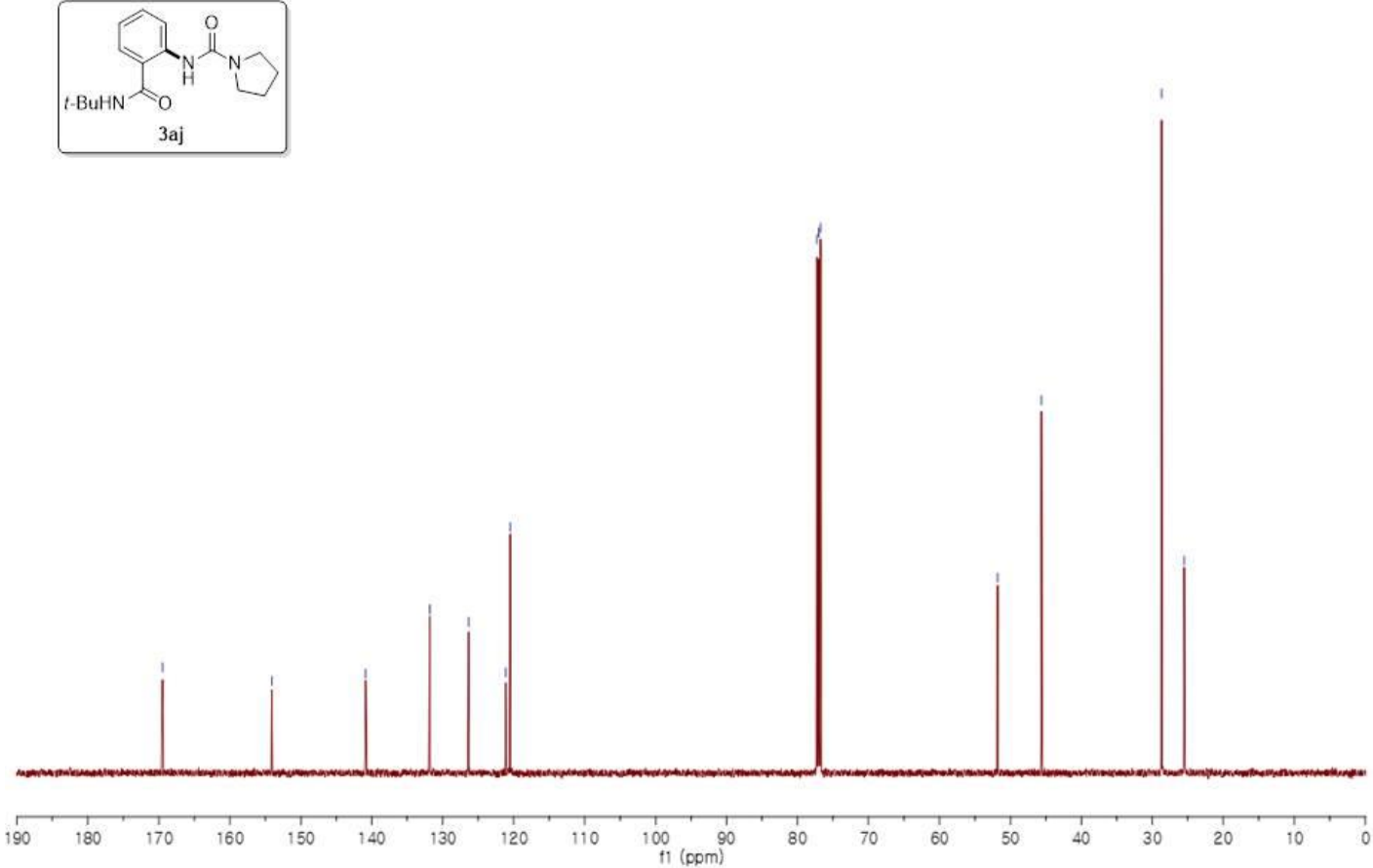


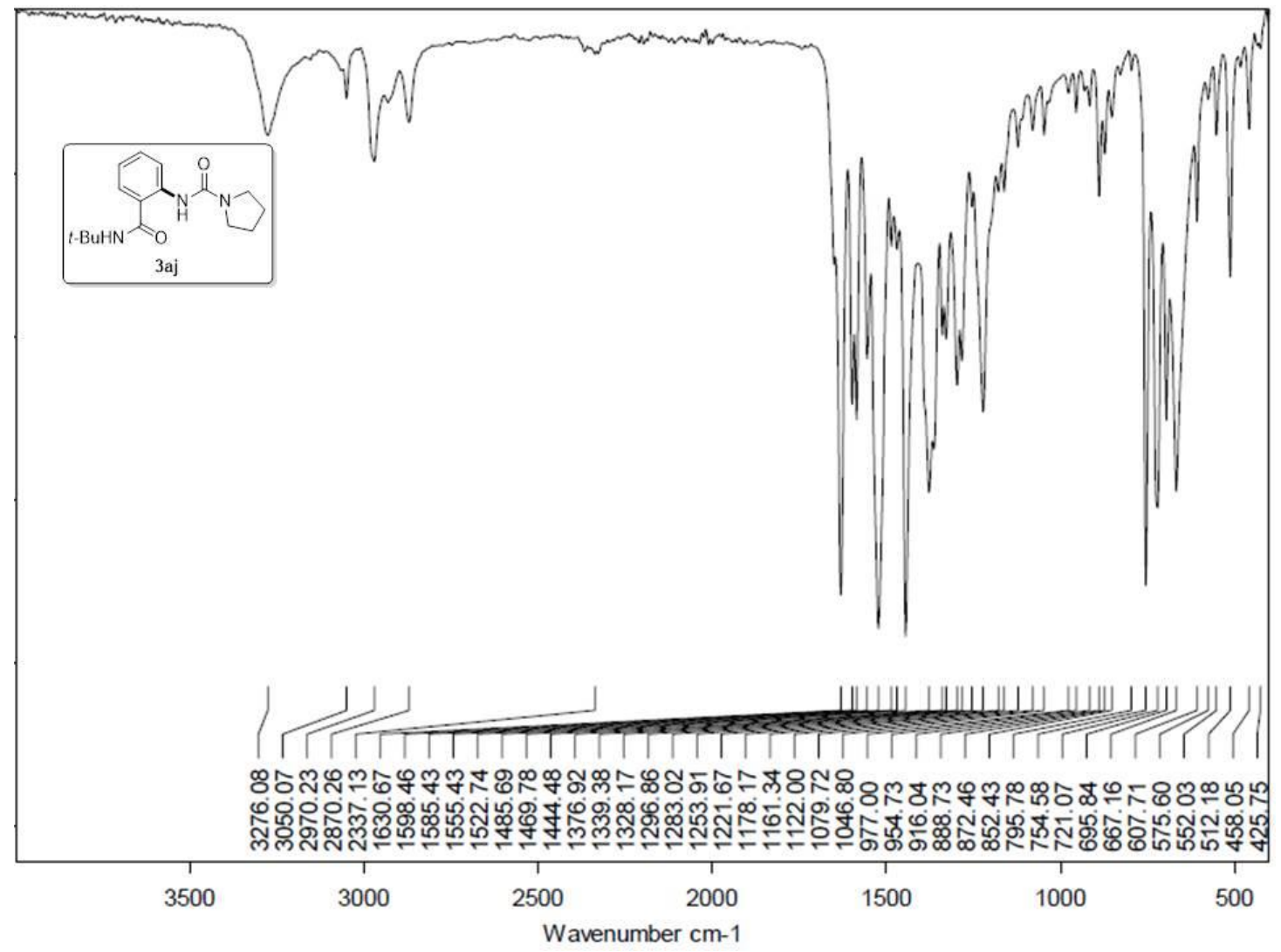


$\mathrm{N}$-(2-(tert-Butylcarbamoyl)phenyl)piperidine-1-carboxamide (Table 2, 3ak, ${ }^{1} \mathrm{H}$ NMR-500 MHz \& ${ }^{13} \mathrm{C}$ NMR-125 MHz in $\mathrm{CDCl}_{3}$ )
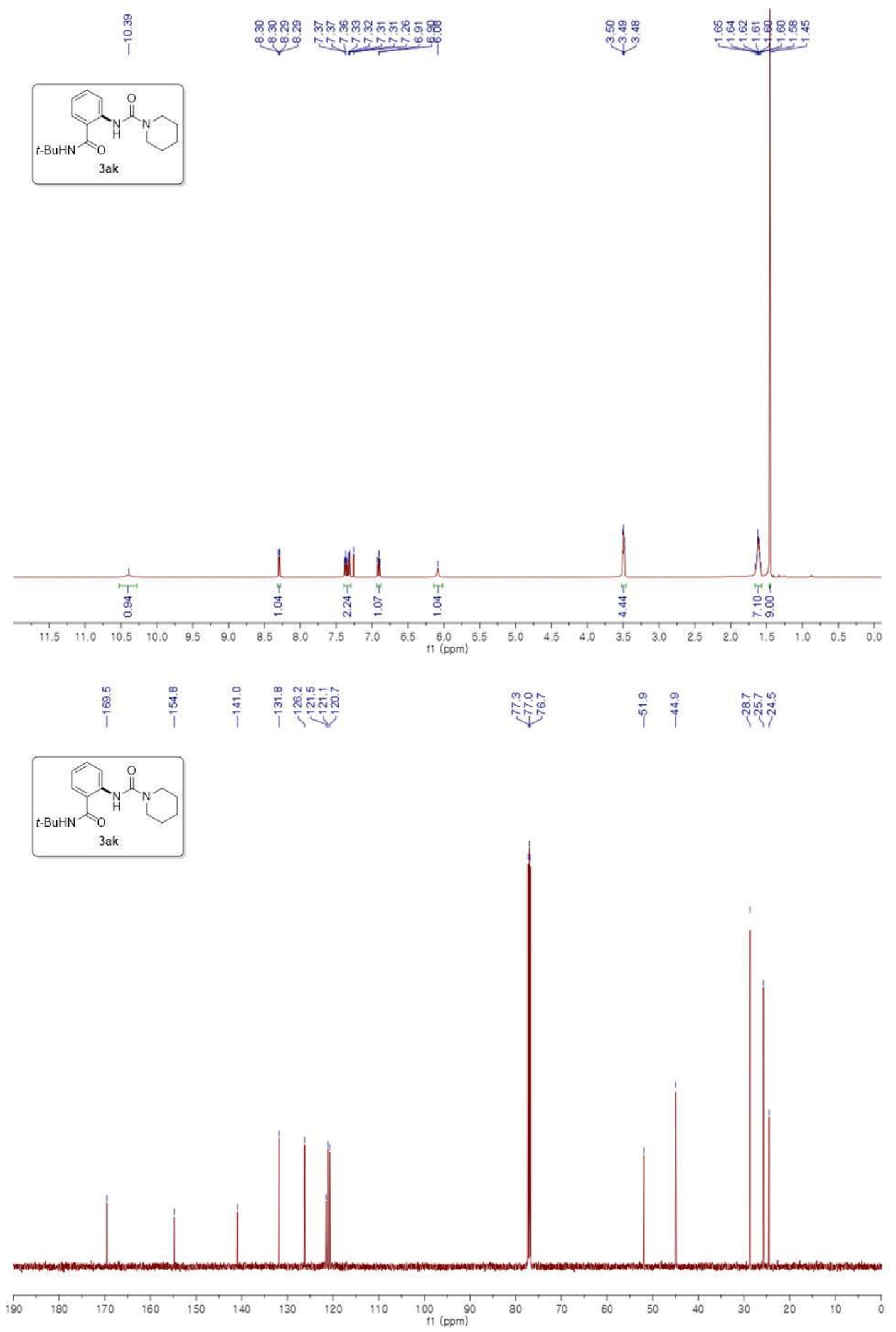


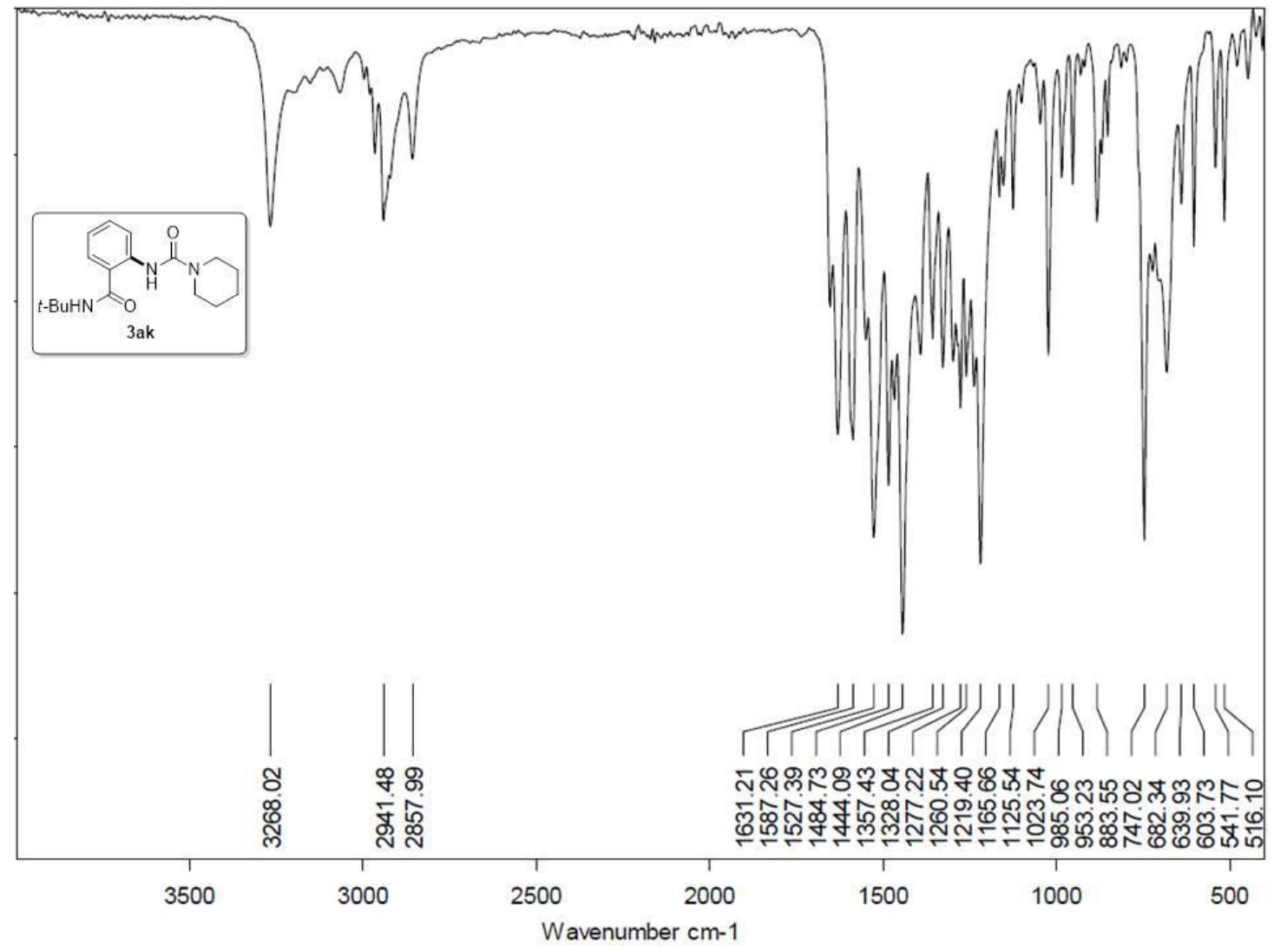


2-(3,3-Diphenylureido)- $\boldsymbol{N}$-isopropylbenzamide (Table 3, 3bc, ${ }^{1} \mathrm{H}$ NMR-500 MHz \& ${ }^{13} \mathrm{C}$ NMR-125 $\mathrm{MHz}$ in Acetone- $\left.d_{6}\right)$
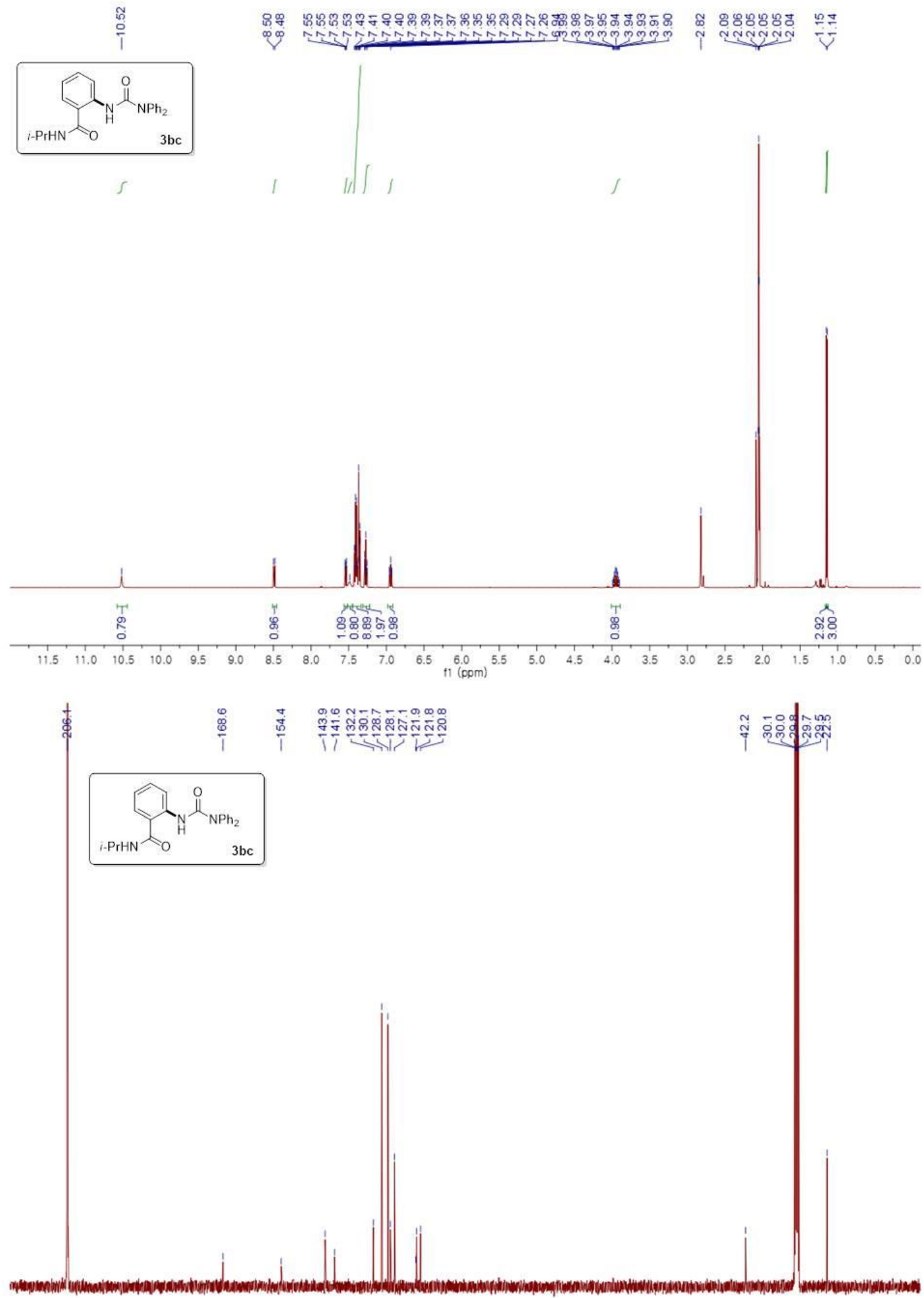

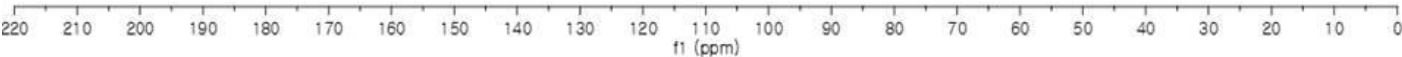




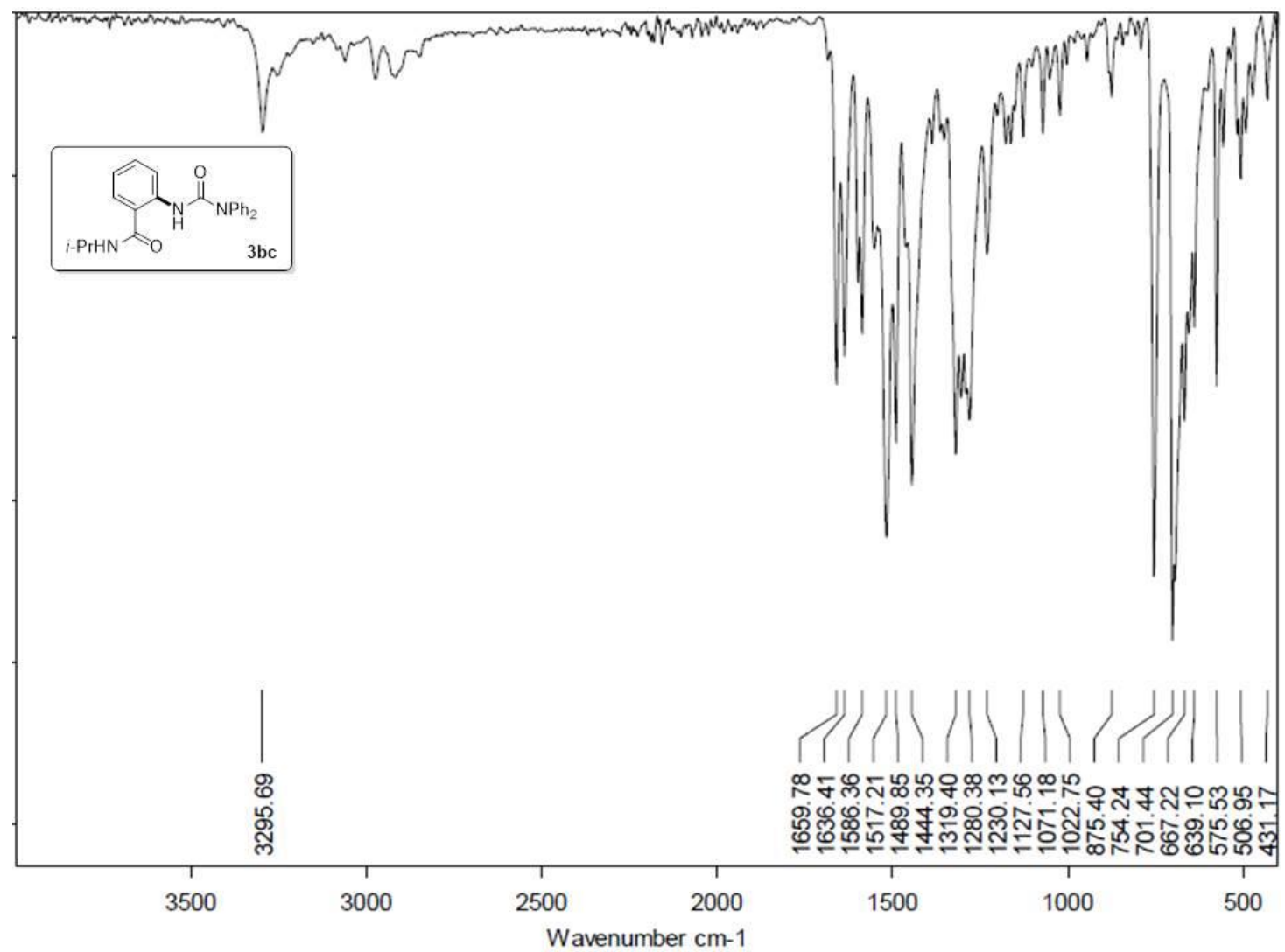


$N$-((1S,2R,5R)-Adamantan-2-yl)-2-(3,3-diphenylureido)benzamide (Table 3, 3cc, ${ }^{1} \mathrm{H}$ NMR-500 $\mathrm{MHz} \&{ }^{13} \mathrm{C}$ NMR-125 MHz in Acetone- $\left.d_{6}\right)$
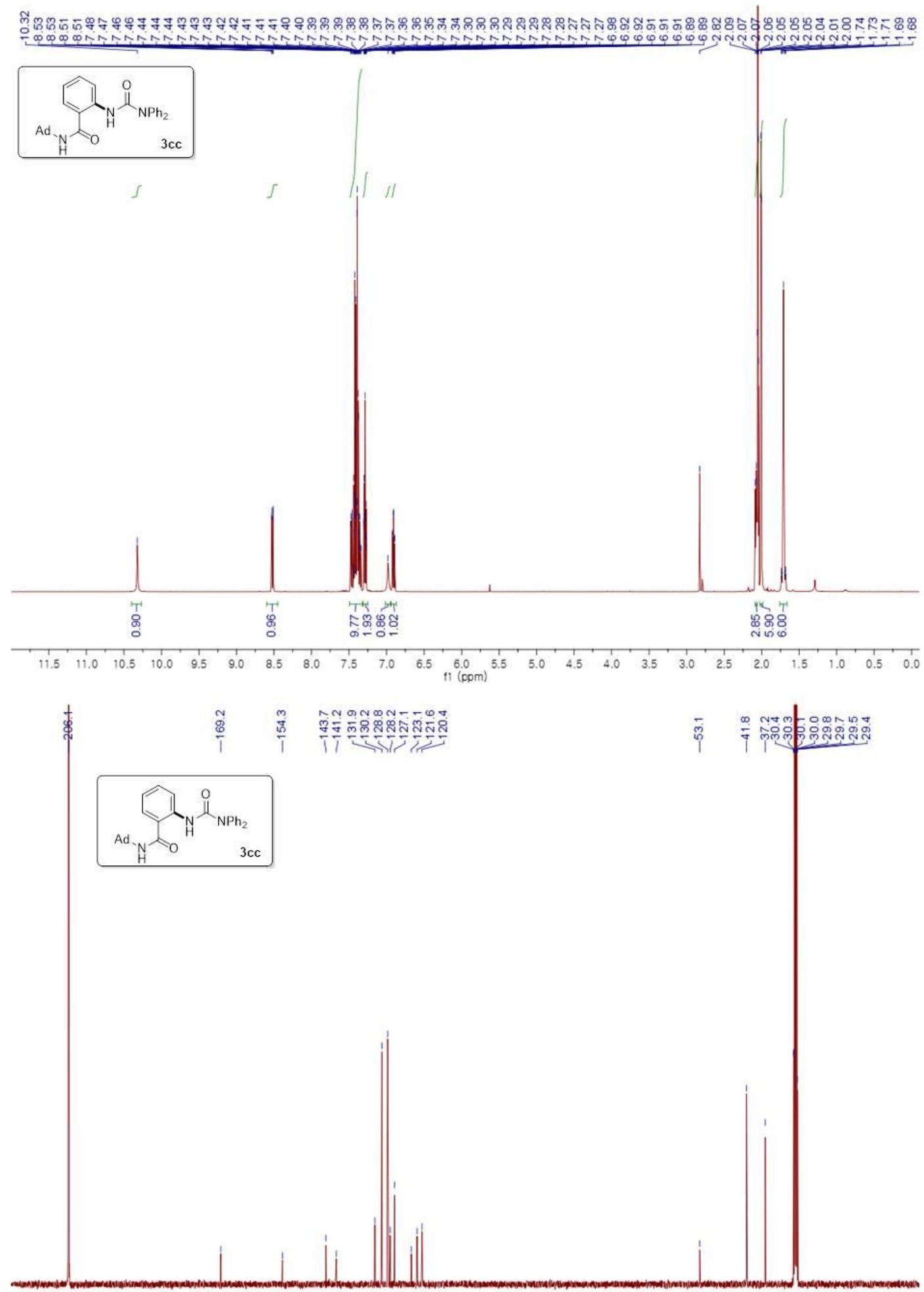

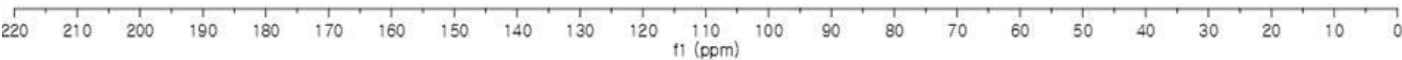




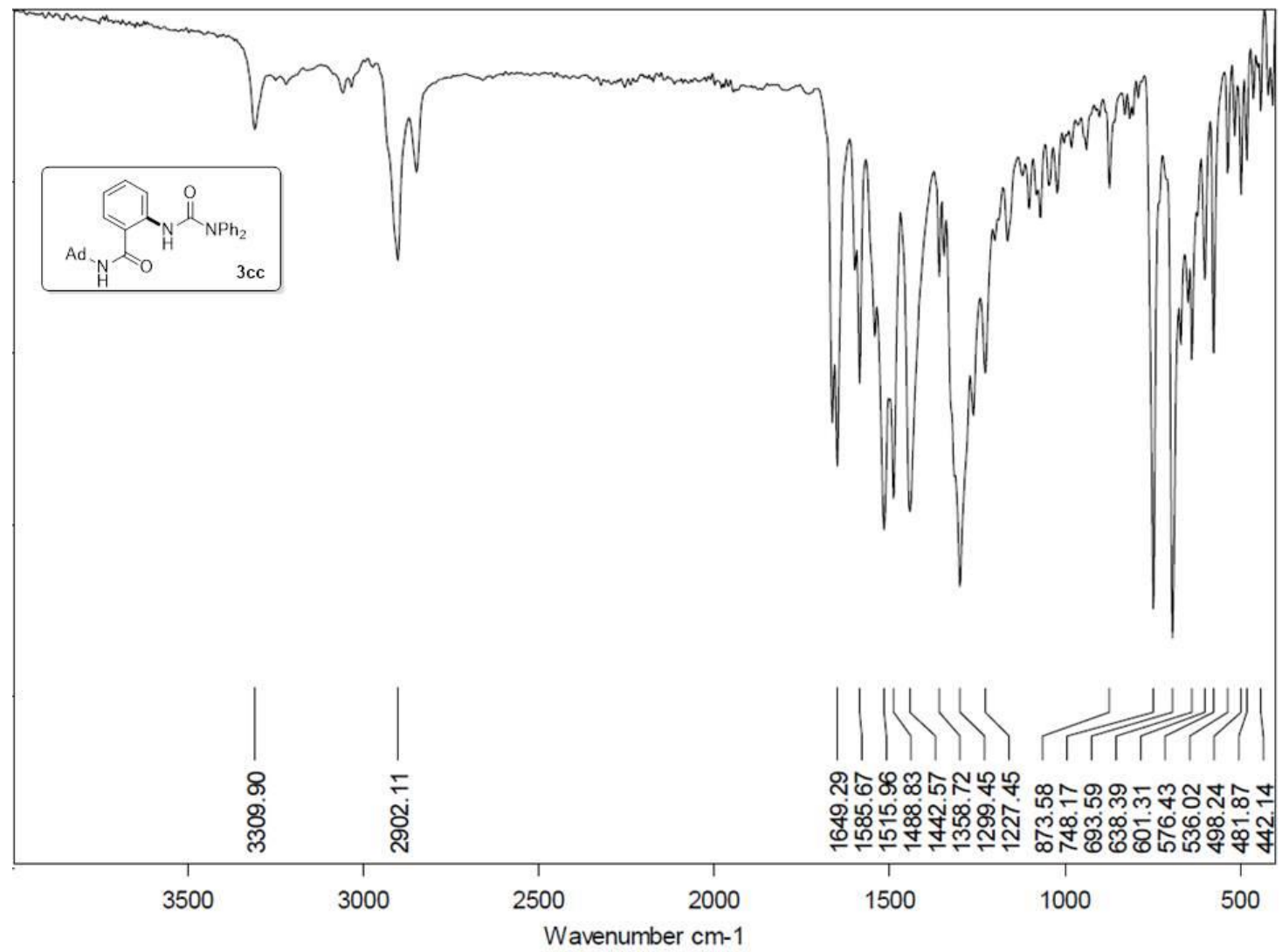


$\mathrm{N}$-(tert-Butyl)-2-(3,3-diphenylureido)-6-fluorobenzamide (Table 3, 3dc, ${ }^{1} \mathrm{H}$ NMR-500 MHz \& ${ }^{13} \mathrm{C}$ NMR-125 MHz in Acetone- $d_{6}$ )

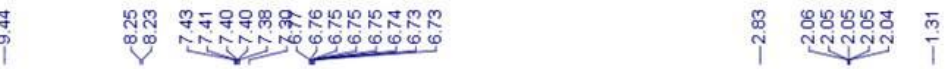
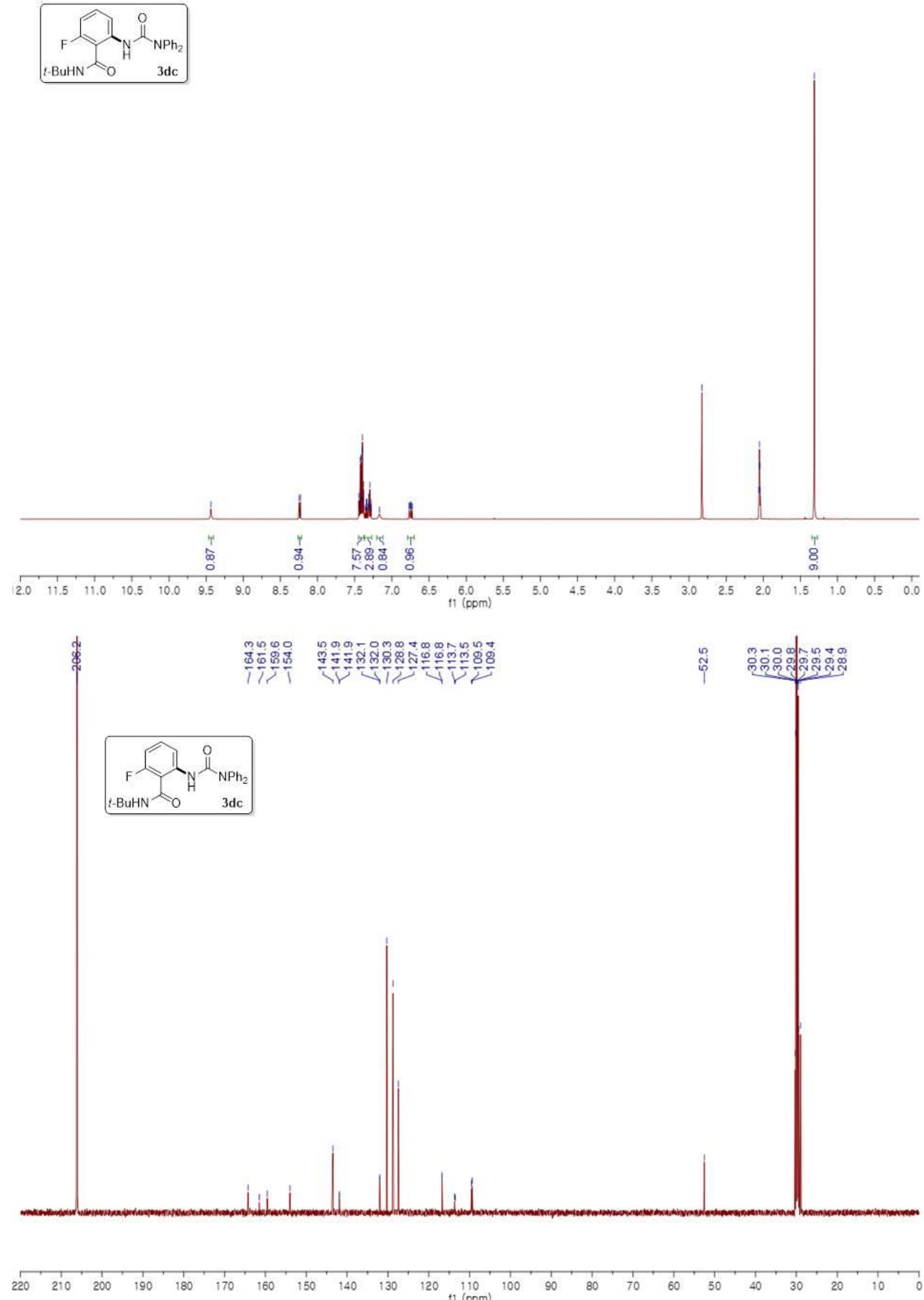


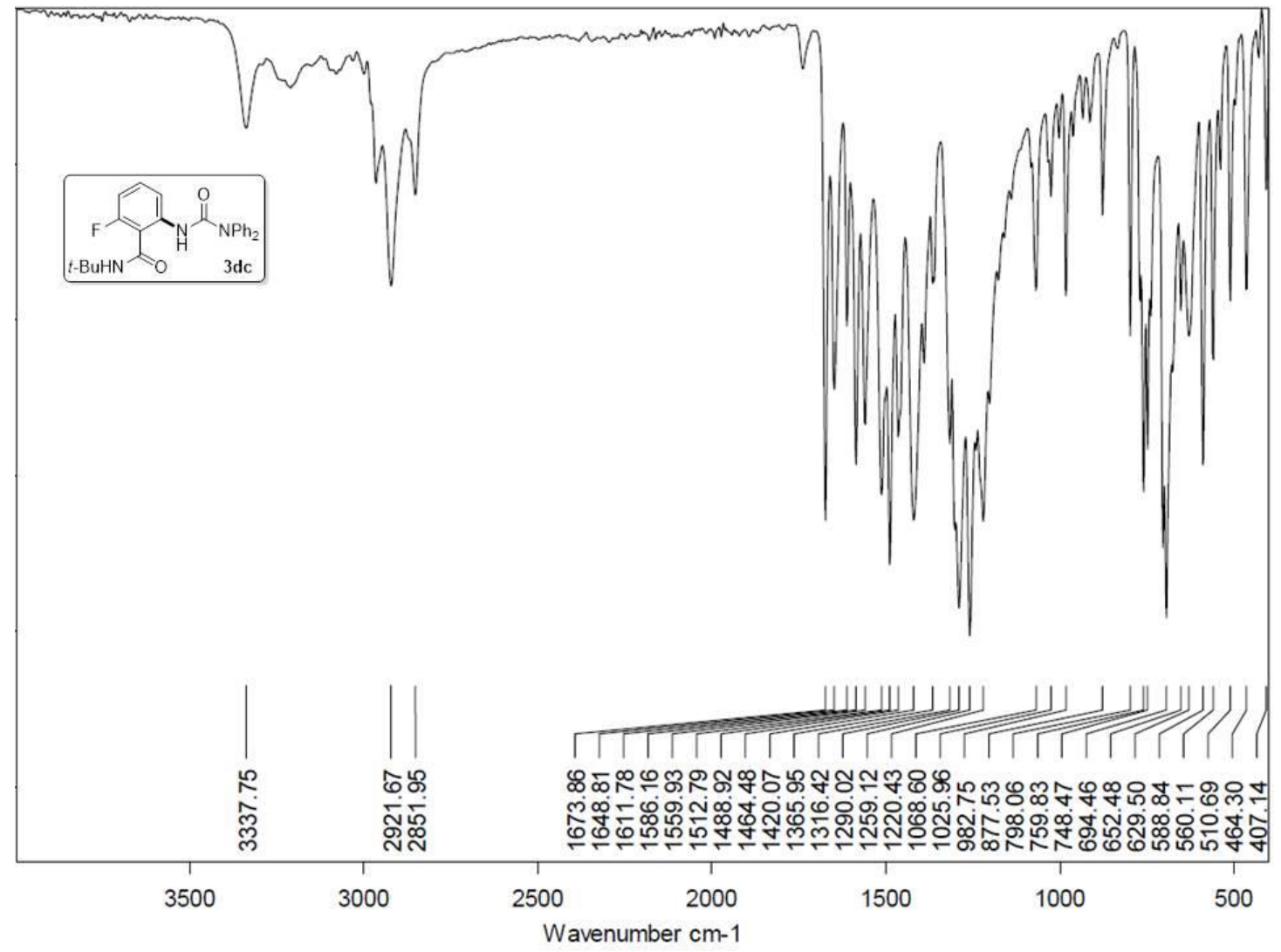


$N$-(tert-Butyl)-2-(3,3-diphenylureido)-4-fluorobenzamide (Table 3, 3ec, ${ }^{1} \mathrm{H}$ NMR-500 MHz \& ${ }^{13} \mathrm{C}$ NMR-125 MHz in Acetone- $\left.d_{6}\right)$
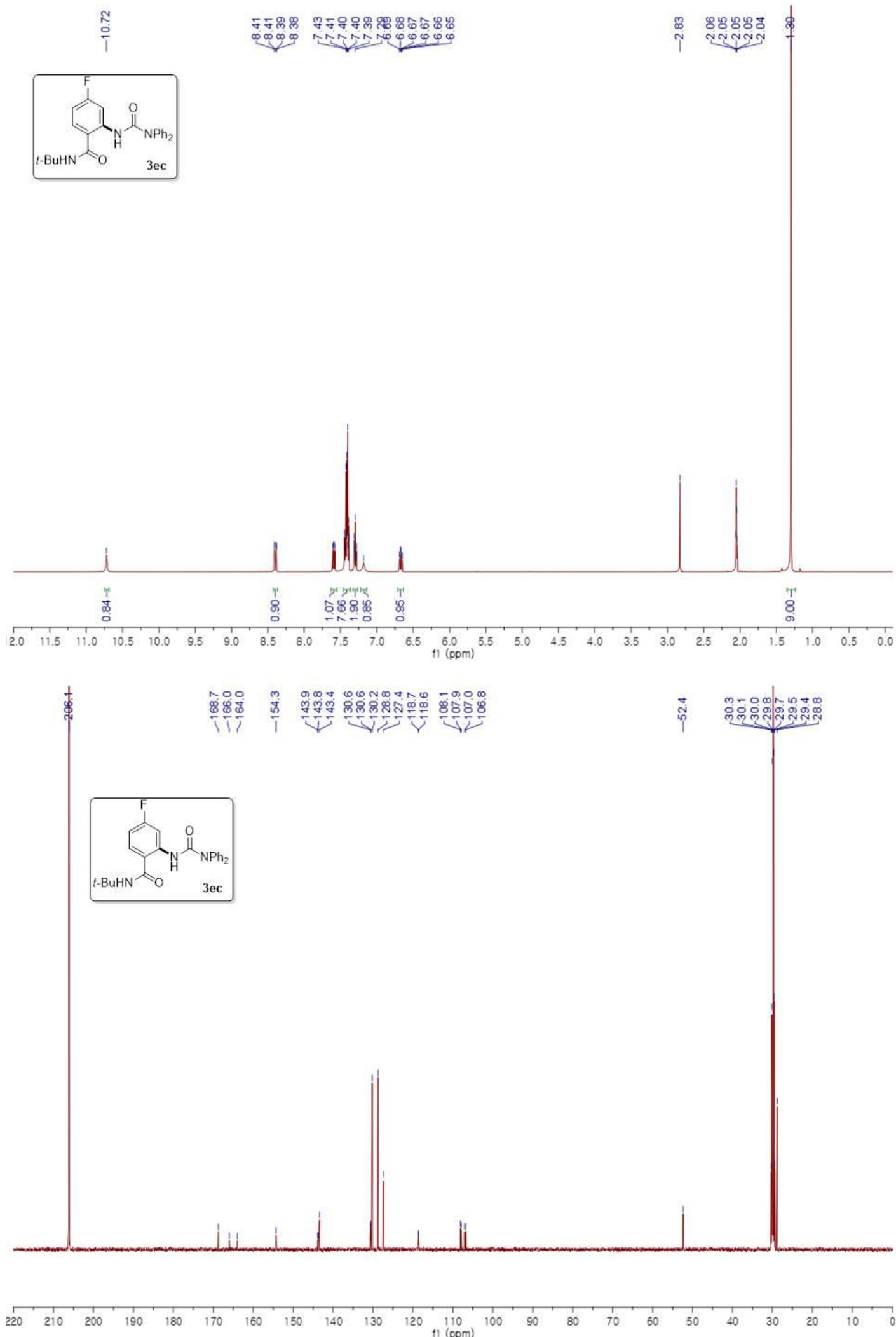


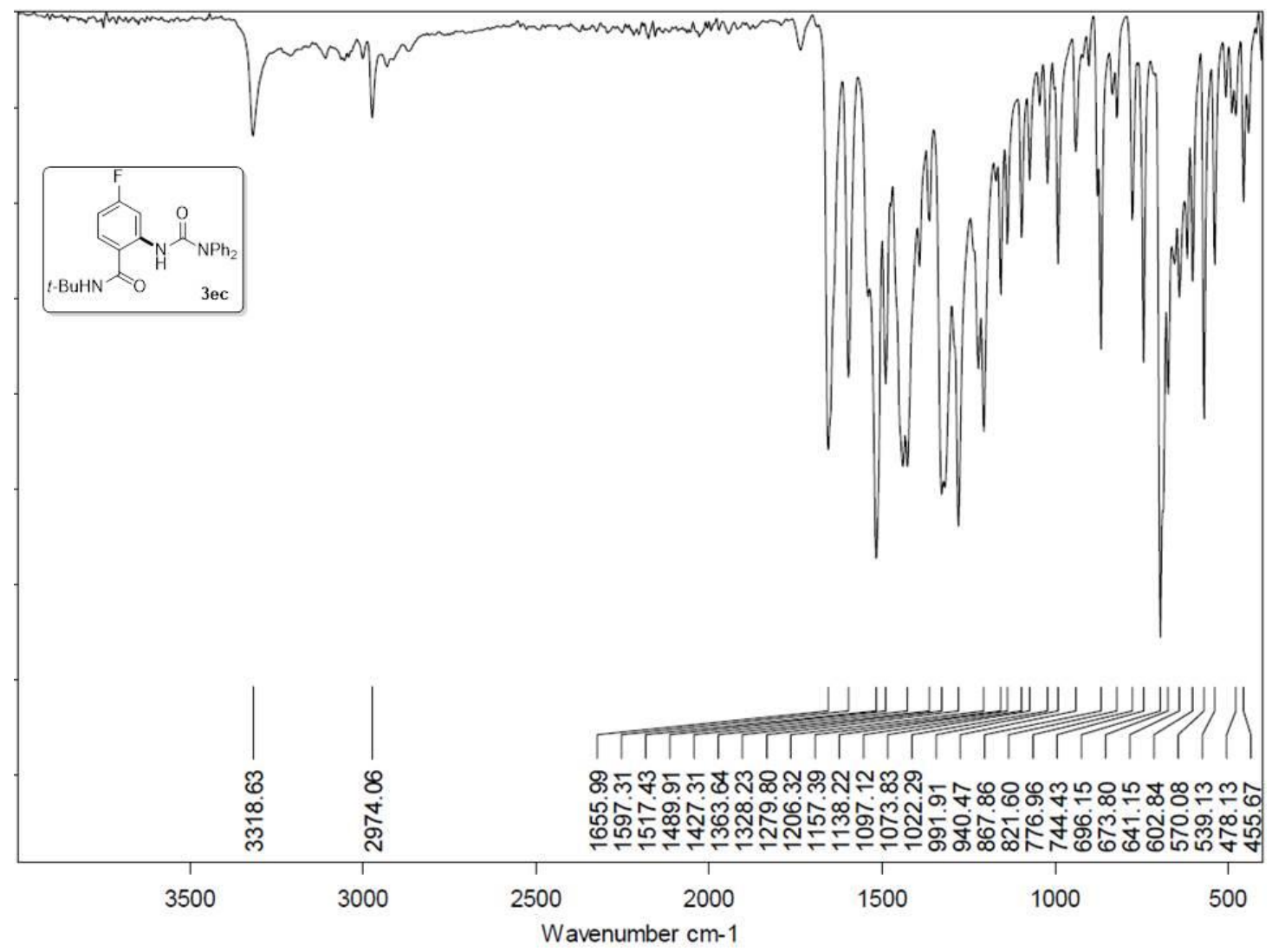


$\mathbf{N}$-(tert-Butyl)-2-(3,3-diphenylureido)-4-(trifluoromethyl)benzamide (Table 3, 3fc, ${ }^{1} \mathrm{H}$ NMR-500 $\mathrm{MHz} \&{ }^{13} \mathrm{C}$ NMR-125 MHz in Acetone- $\left.d_{6}\right)$
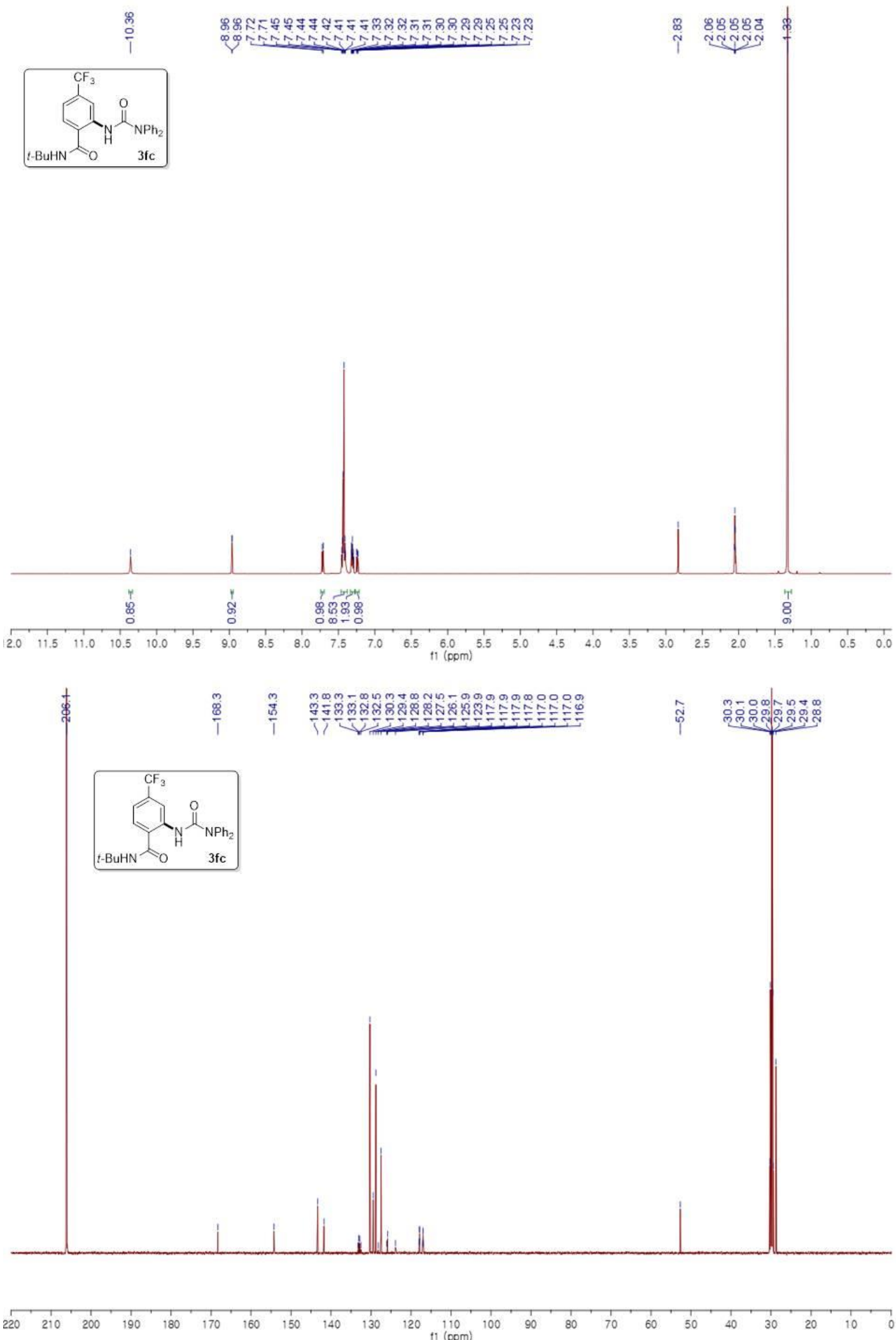


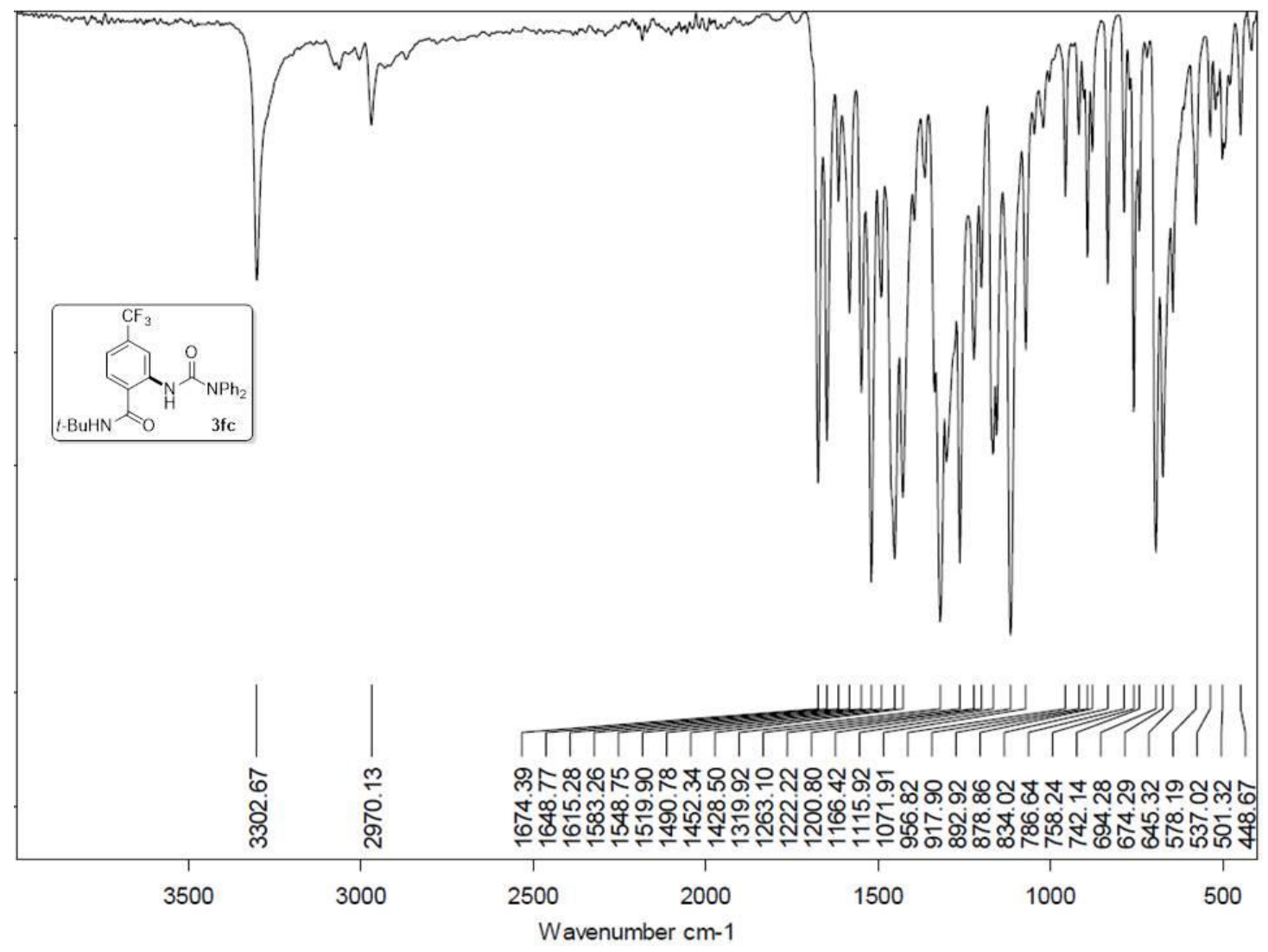


4-Bromo- $N$-(tert-butyl)-2-(3,3-diphenylureido)benzamide (Table 3, 3gc, ${ }^{1} \mathrm{H}$ NMR-500 MHz \& ${ }^{13} \mathrm{C}$ NMR-125 MHz in Acetone- $\left.d_{6}\right)$
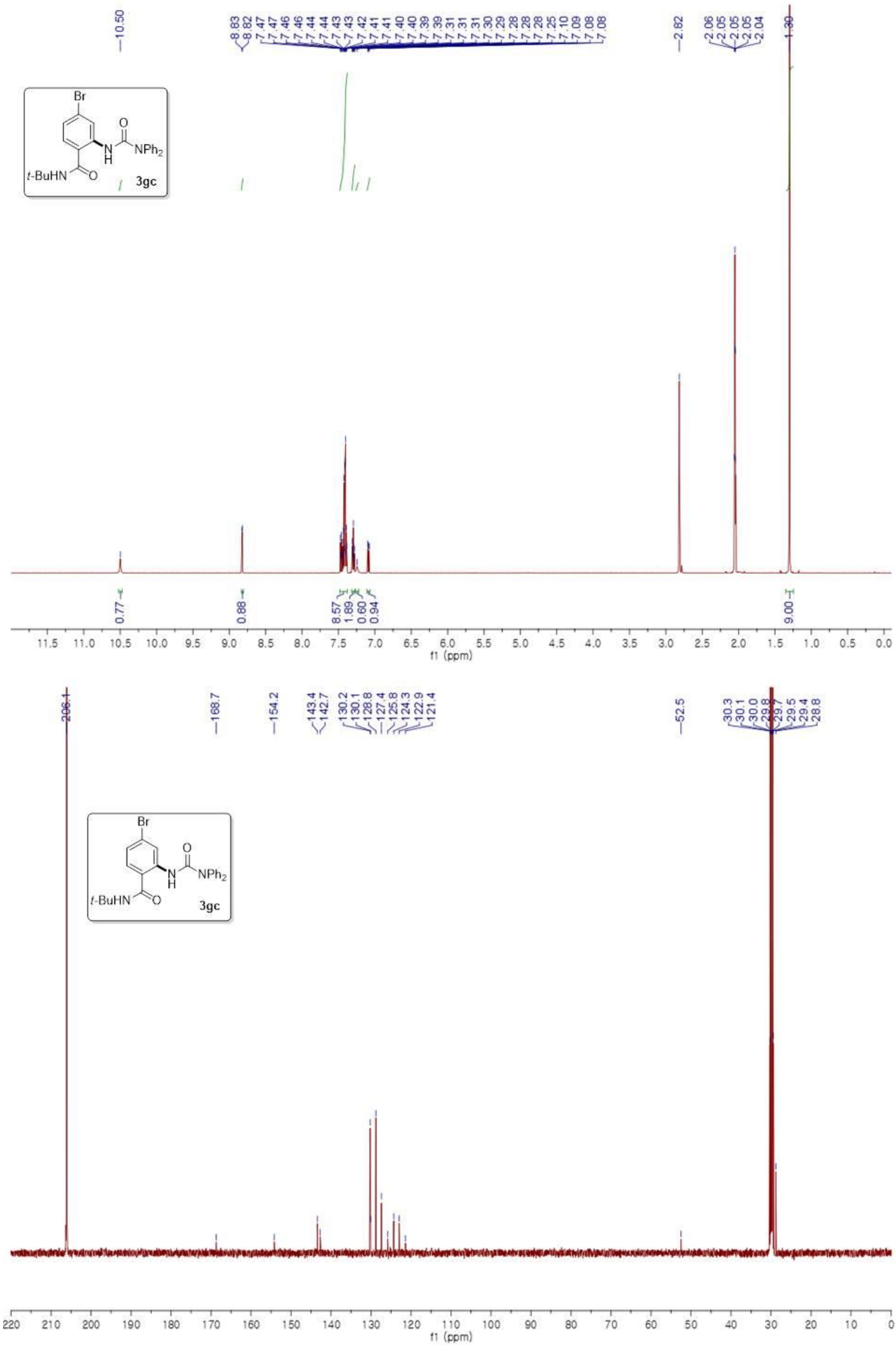


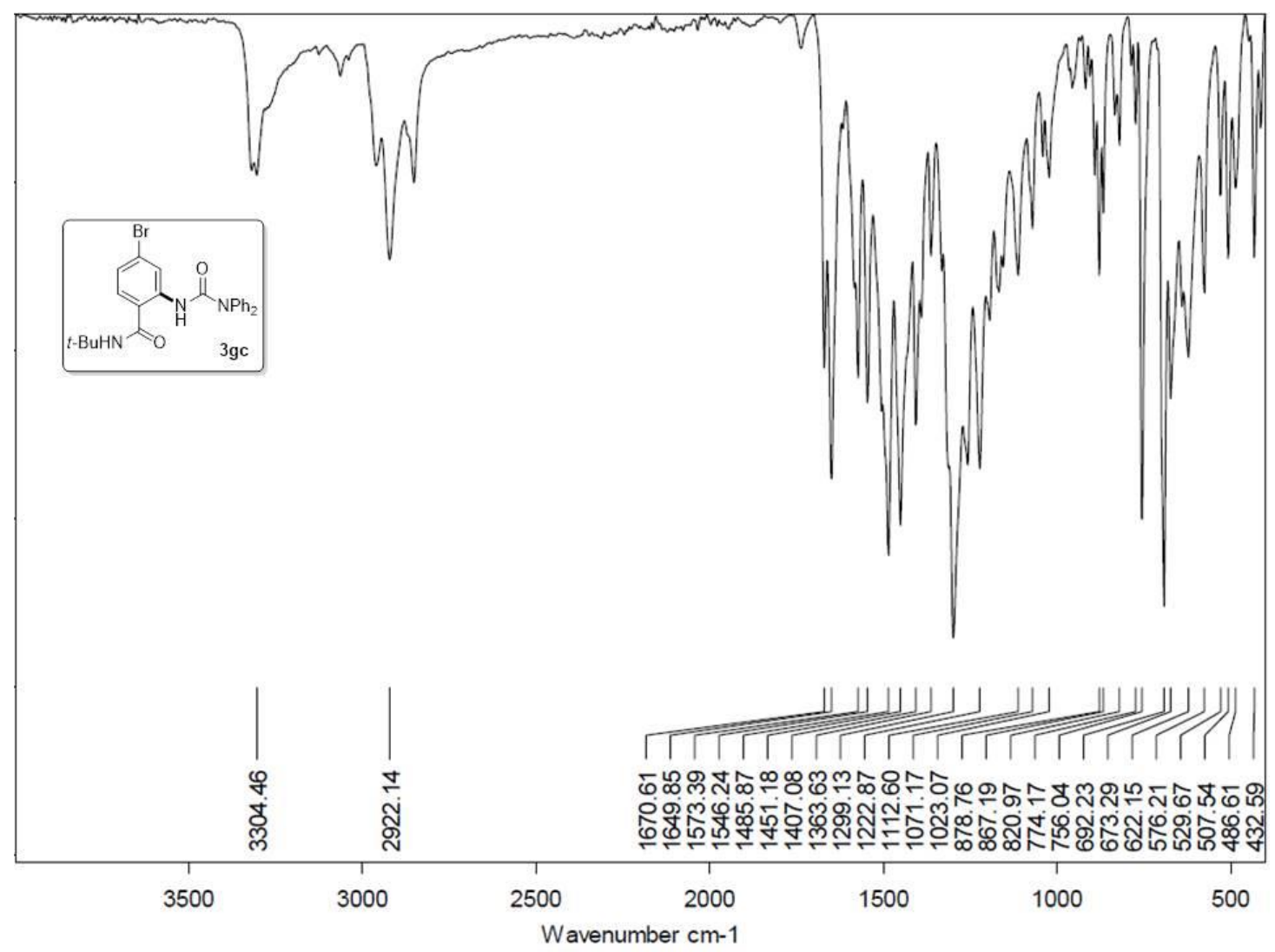


5-Bromo- $N$-(tert-butyl)-2-(3,3-diphenylureido)benzamide (Table 3,3 he, ${ }^{1} \mathrm{H}$ NMR-500 MHz \& ${ }^{13} \mathrm{C}$ NMR-125 MHz in Acetone- $\left.d_{6}\right)$
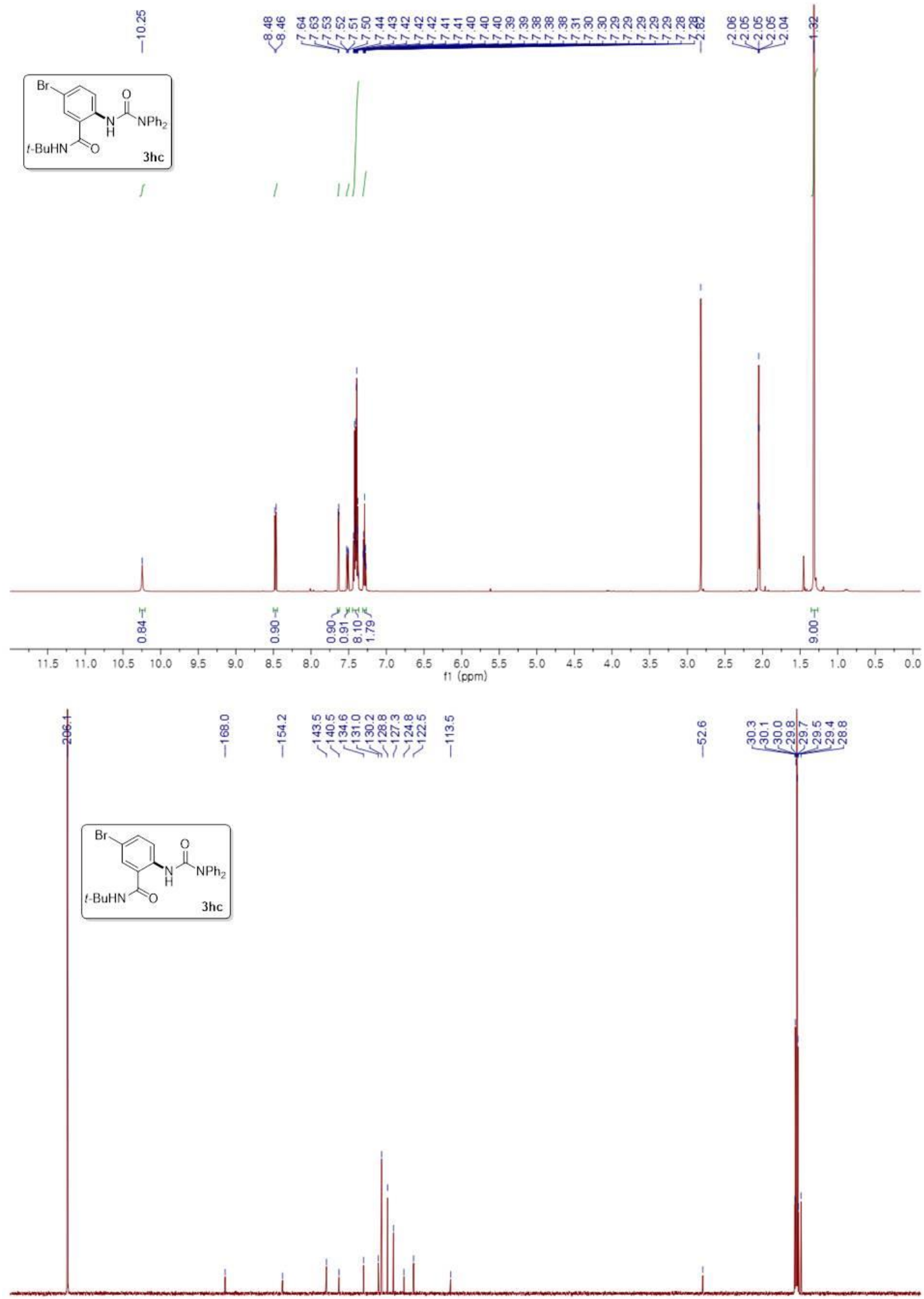

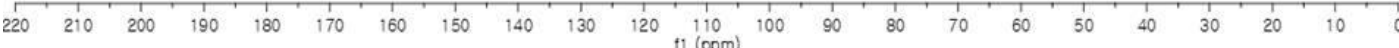




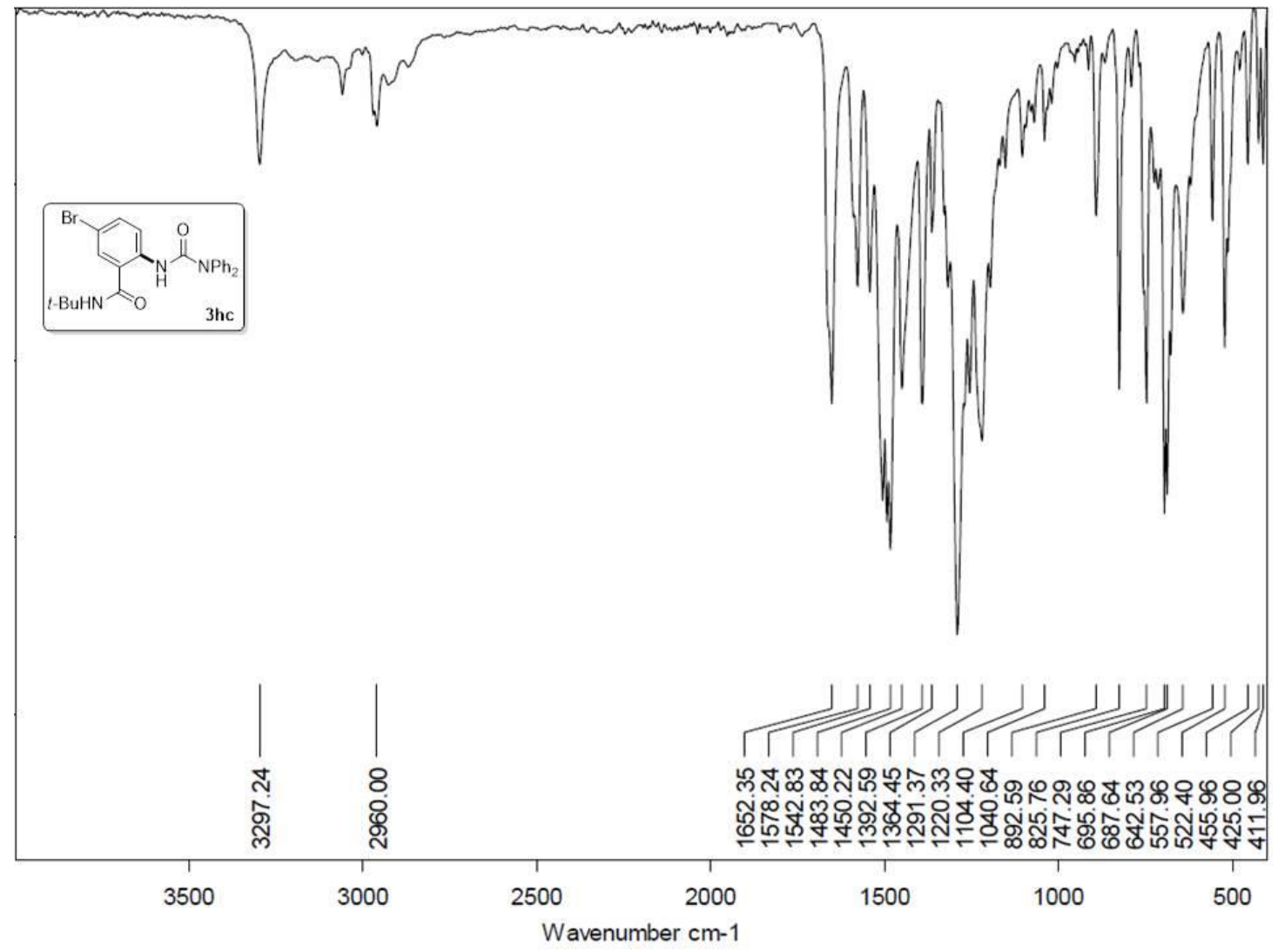


$\mathrm{N}$-(tert-Butyl)-2-(3,3-diphenylureido)-4-iodobenzamide (Table 3, 3ic, ${ }^{1} \mathrm{H}$ NMR-500 MHz \& ${ }^{13} \mathrm{C}$ NMR-125 MHz in Acetone- $d_{6}$ )
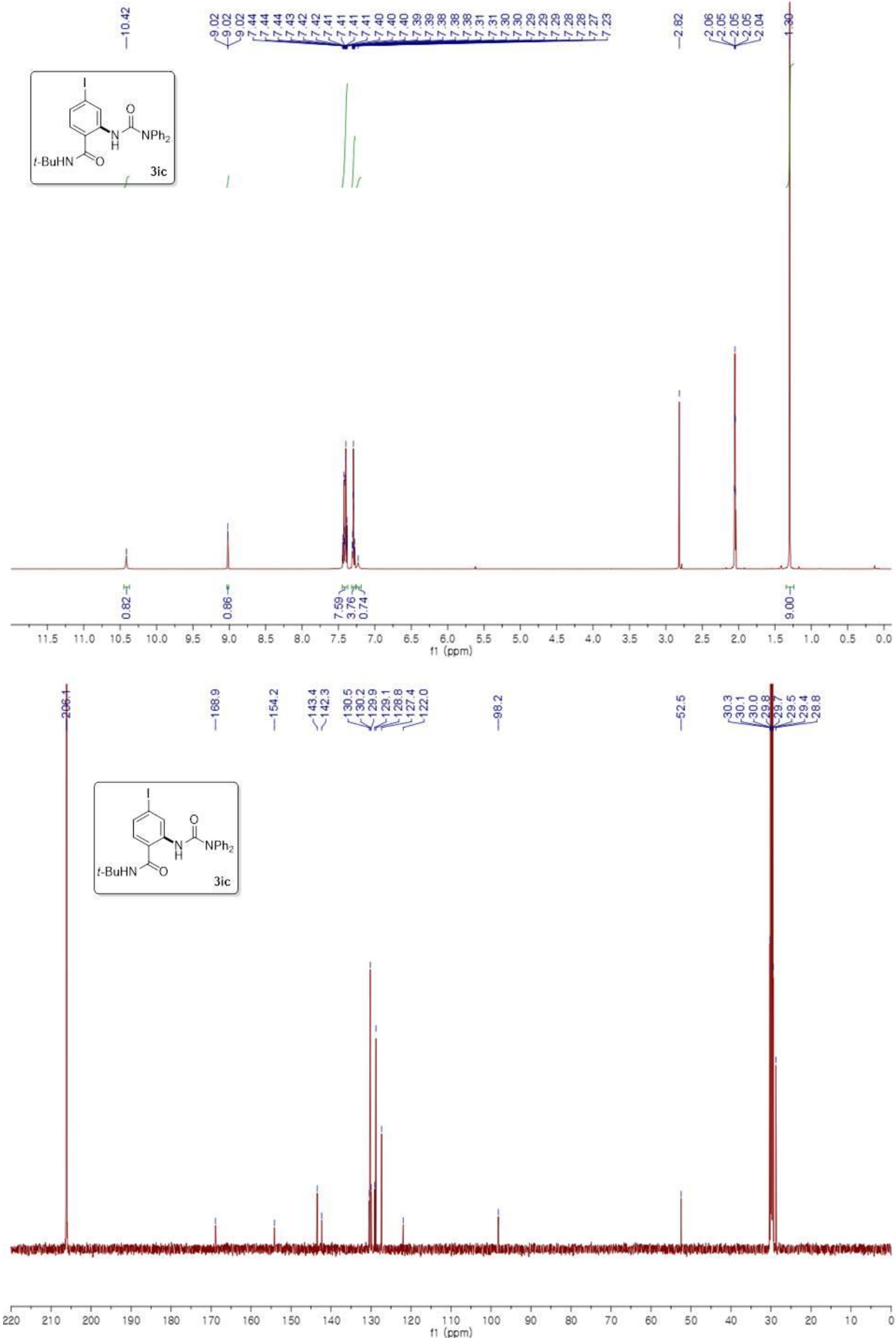


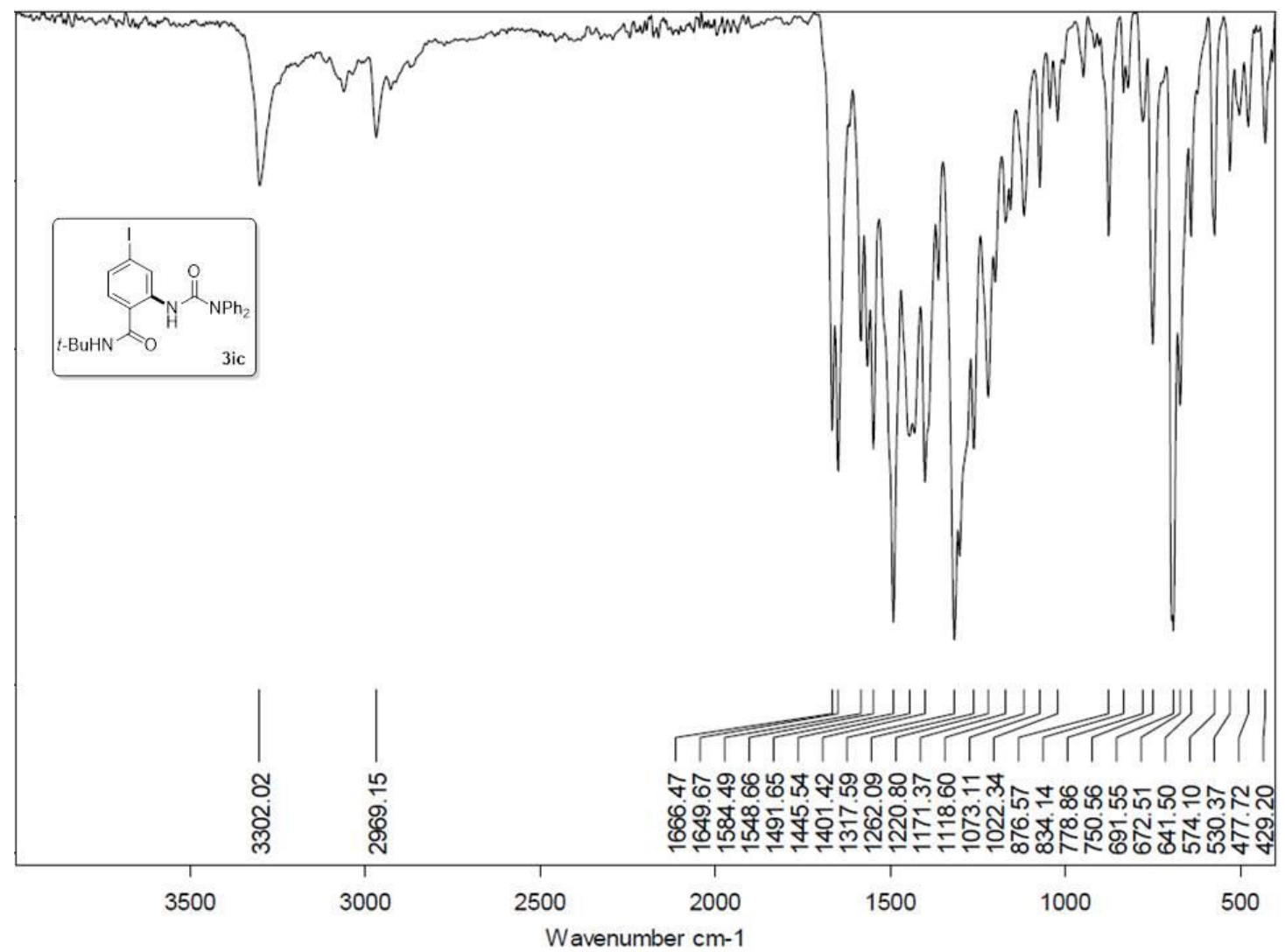


$\mathrm{N}$-(tert-Butyl)-2-(3,3-diphenylureido)-6-methylbenzamide (Table 3, 3jc, ${ }^{1} \mathrm{H}$ NMR-500 MHz \& ${ }^{13} \mathrm{C}$ NMR-125 MHz in Acetone- $\left.d_{6}\right)$

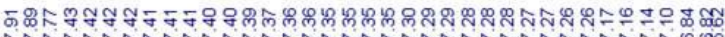

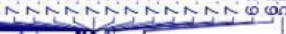

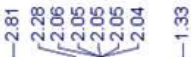

t-BuHN
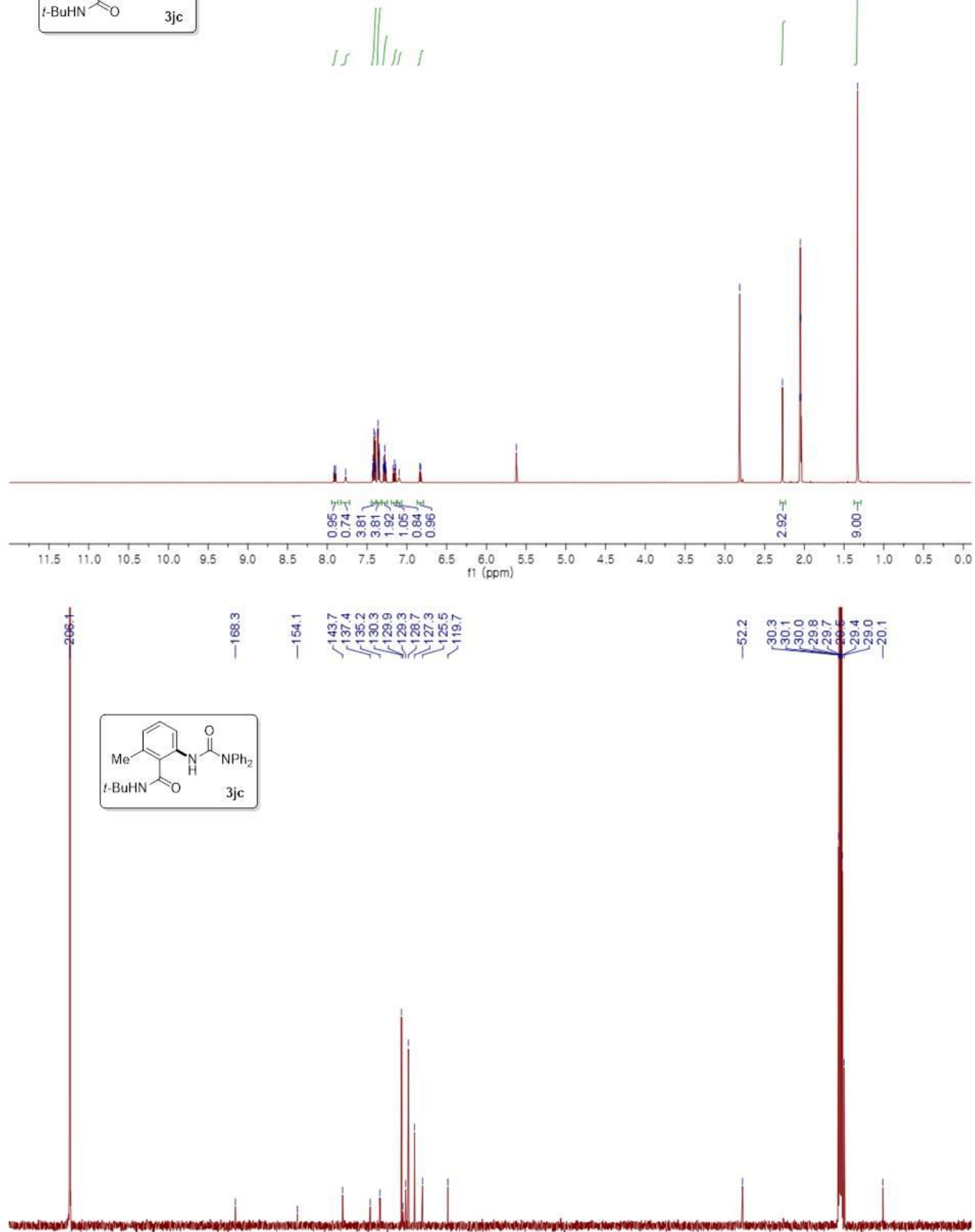

$\begin{array}{llllllllllllllllllllllllllllllllllllllllllll}220 & 210 & 200 & 190 & 180 & 170 & 160 & 150 & 140 & 130 & 120 & 110 & 100 & 90 & 80 & 70 & 60 & 50 & 40 & 30 & 20 & 10 & 0\end{array}$ 


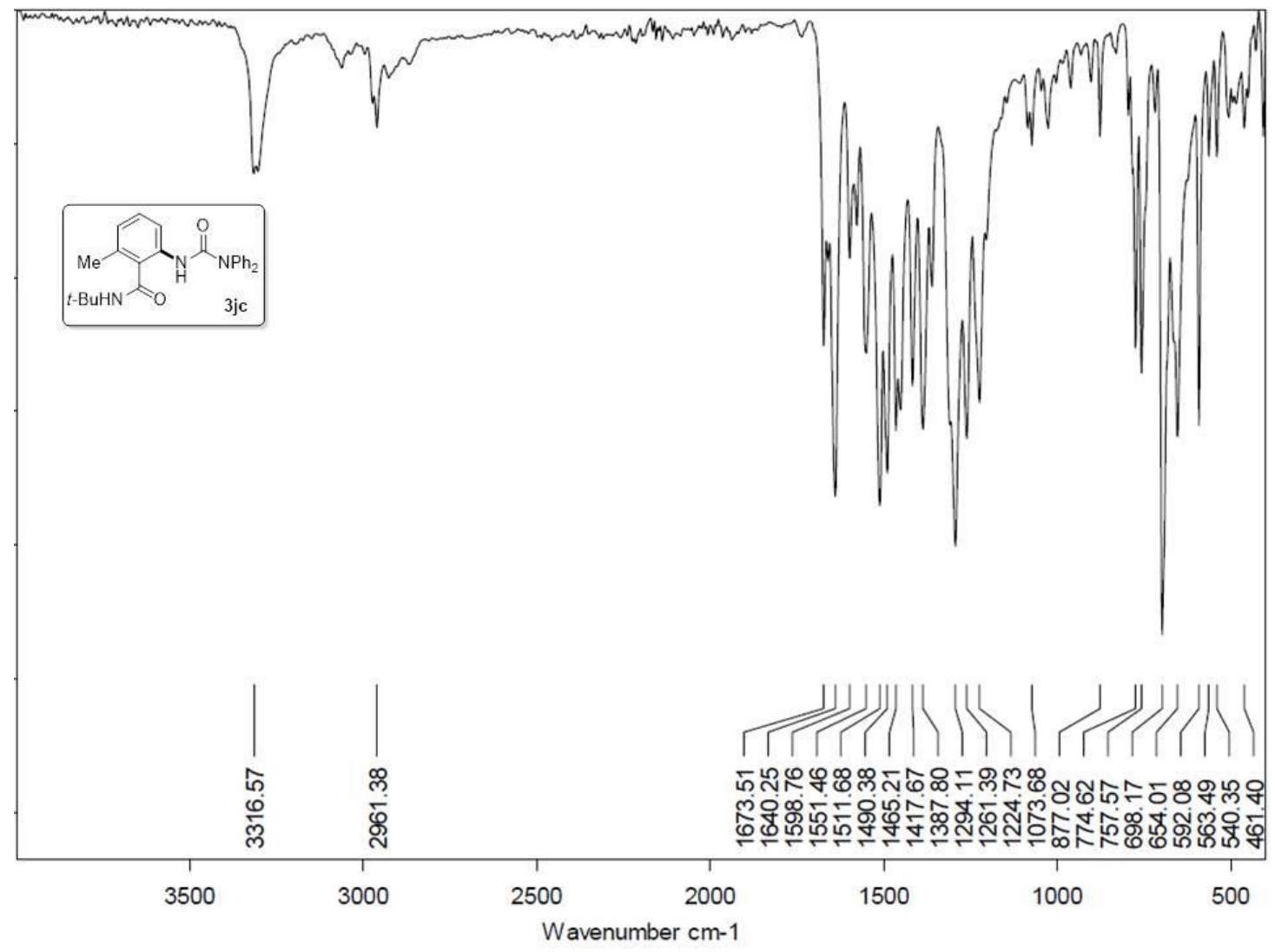


$\boldsymbol{N}$-(tert-Butyl)-2-(3,3-diphenylureido)-5-methylbenzamide (Table 3, 3kc, ${ }^{1} \mathrm{H}$ NMR-500 MHz \& ${ }^{13} \mathrm{C}$ NMR-125 MHz in Acetone- $\left.d_{6}\right)$
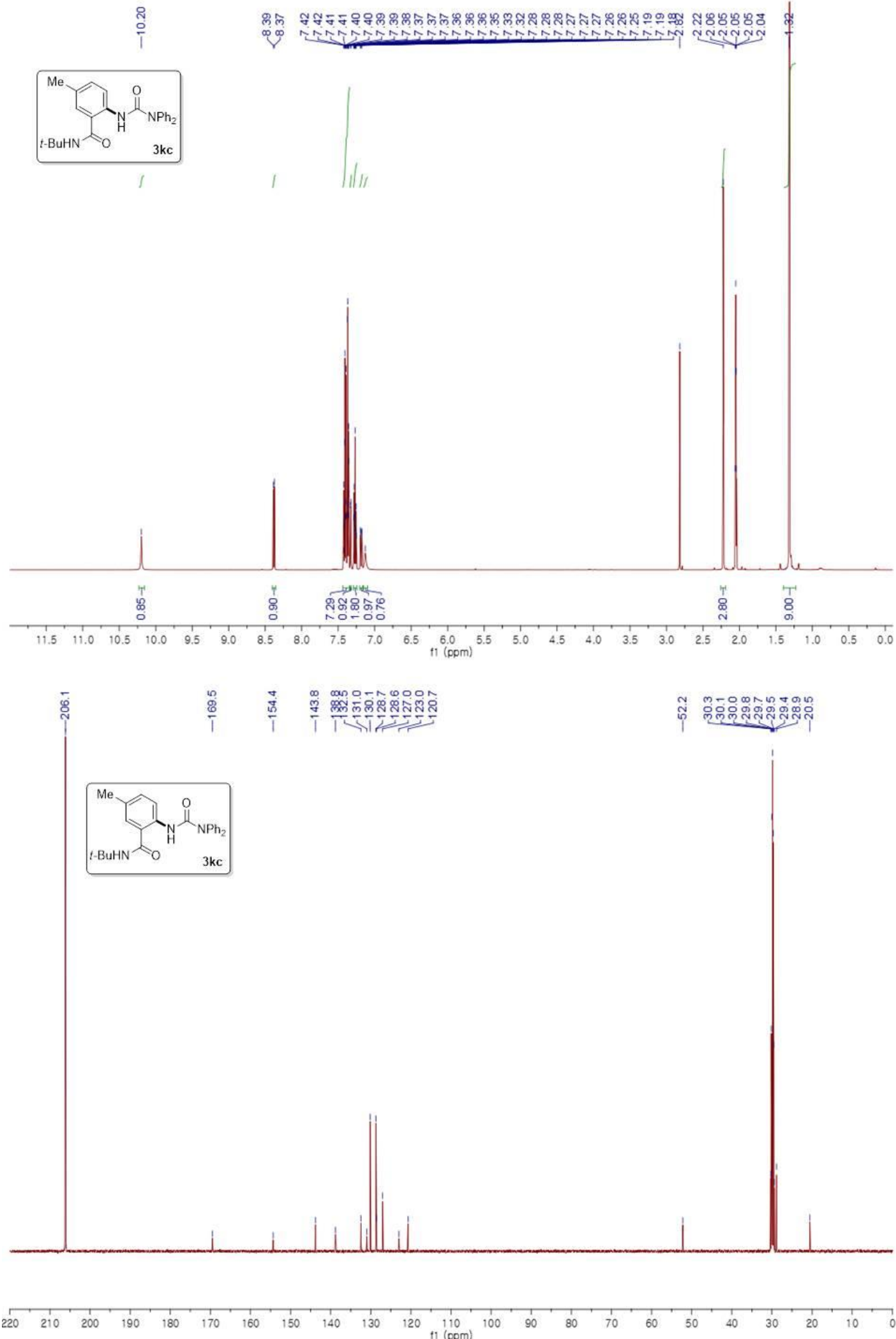


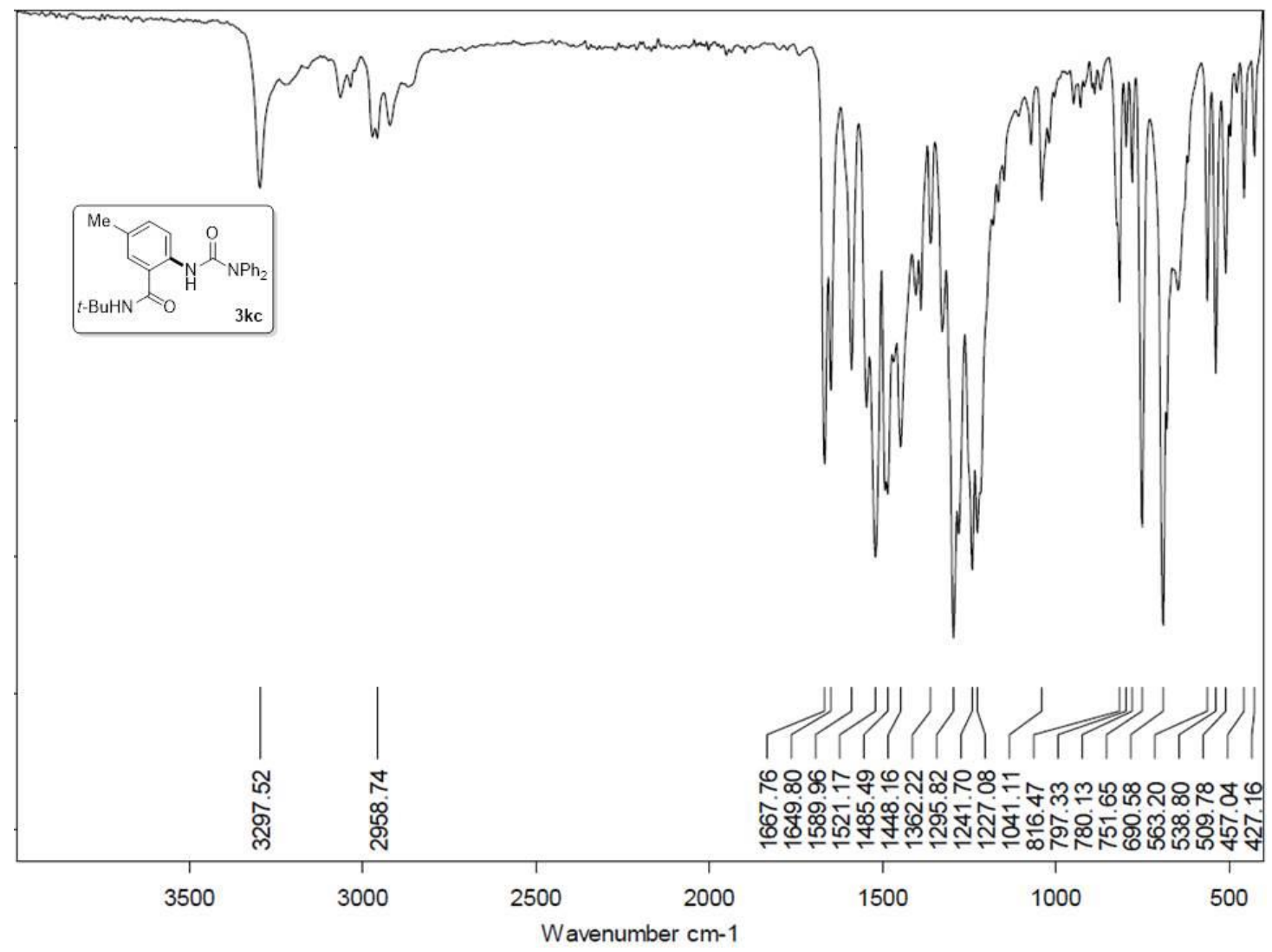


$\mathrm{N}$-(tert-Butyl)-2-(3,3-diphenylureido)-4-methylbenzamide (Table 3, 3lc, ${ }^{1} \mathrm{H} \mathrm{NMR}-500 \mathrm{MHz} \&{ }^{13} \mathrm{C}$ NMR-125 MHz in Acetone- $\left.d_{6}\right)$
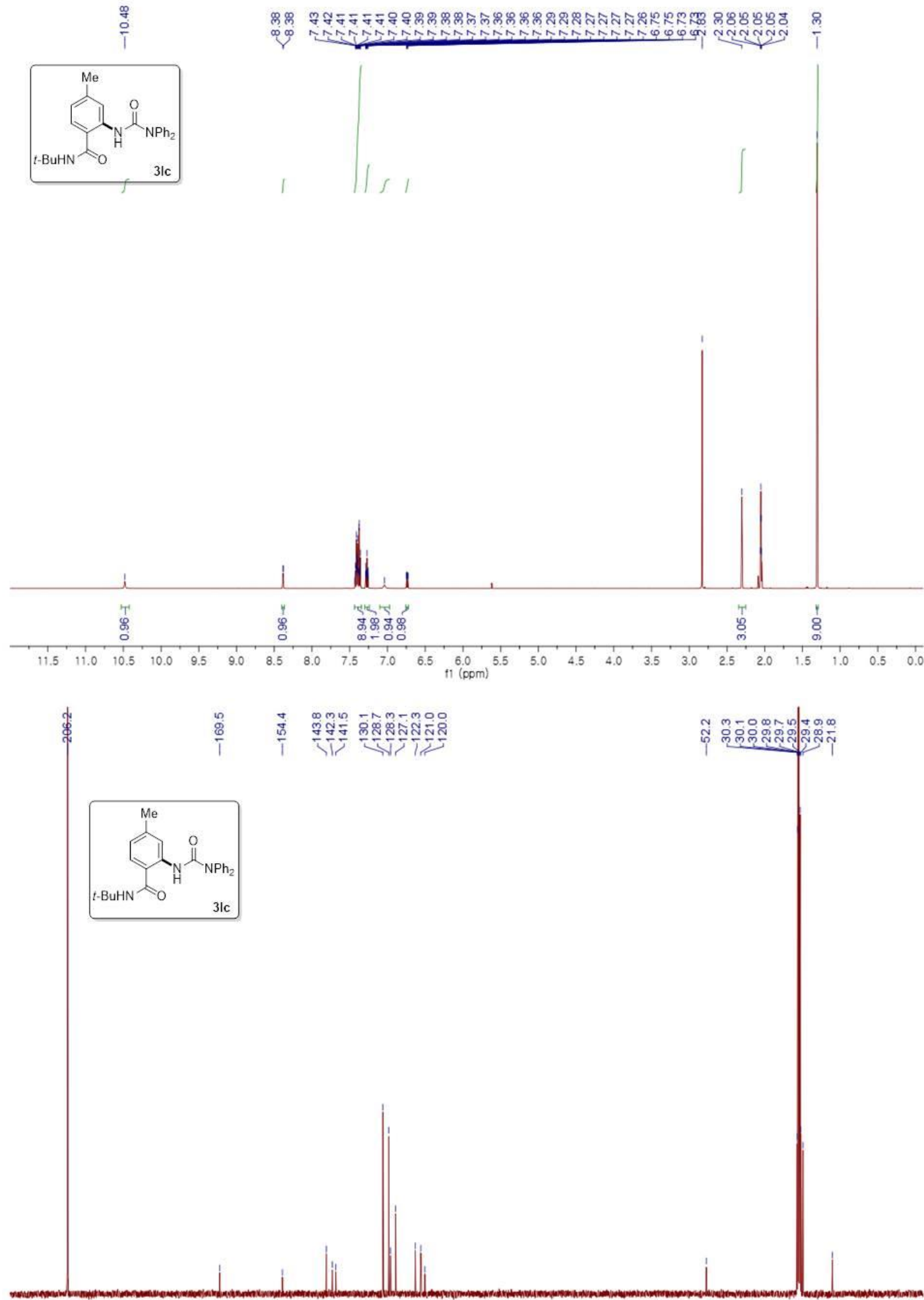

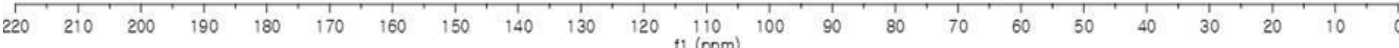




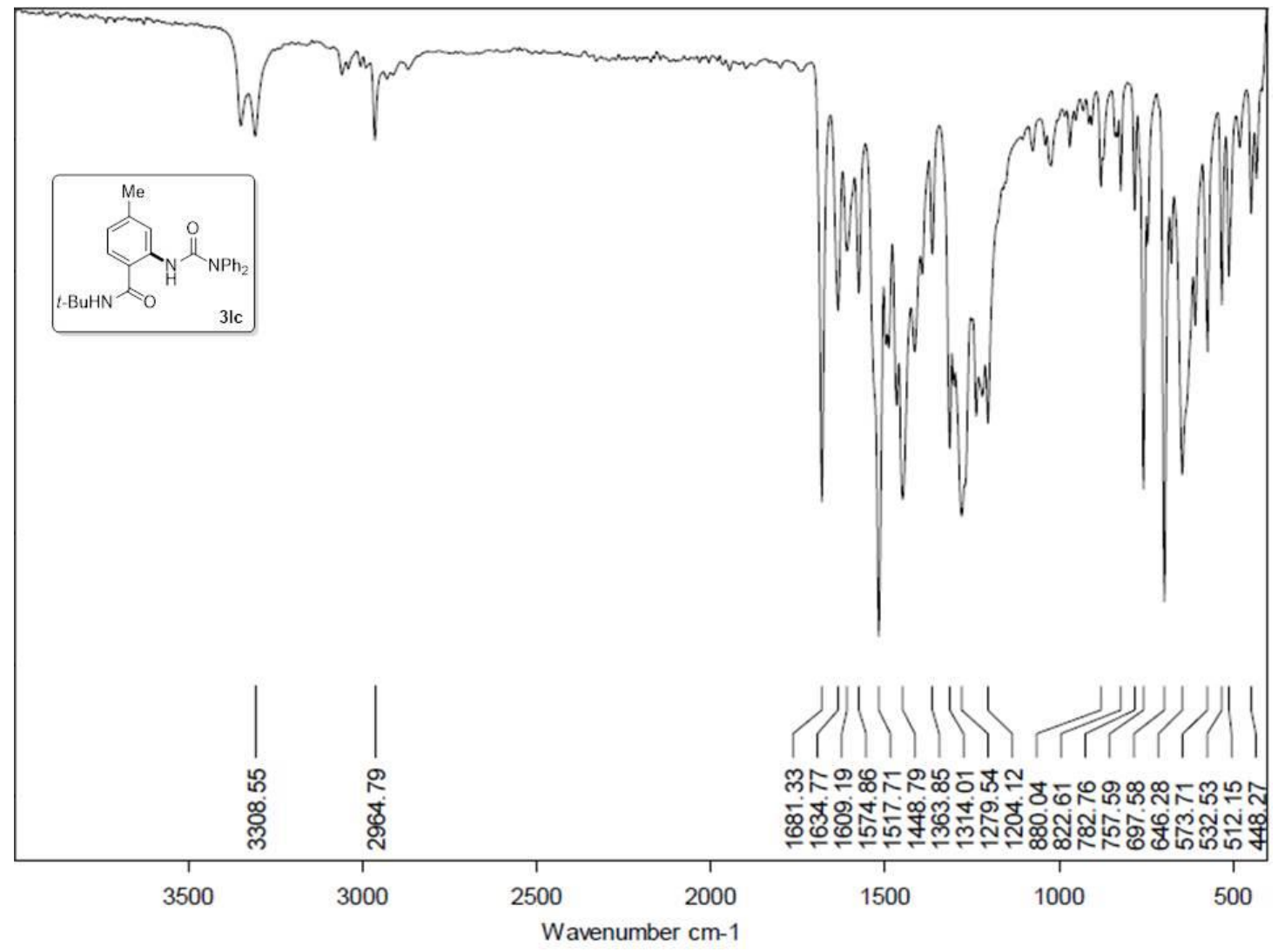


$\mathrm{N}$-(tert-Butyl)-2-(3,3-diphenylureido)-4-methoxybenzamide (Table 3, 3mc, ${ }^{1} \mathrm{H}$ NMR-500 MHz \& ${ }^{13} \mathrm{C}$ NMR-125 MHz in Acetone- $\left.d_{6}\right)$
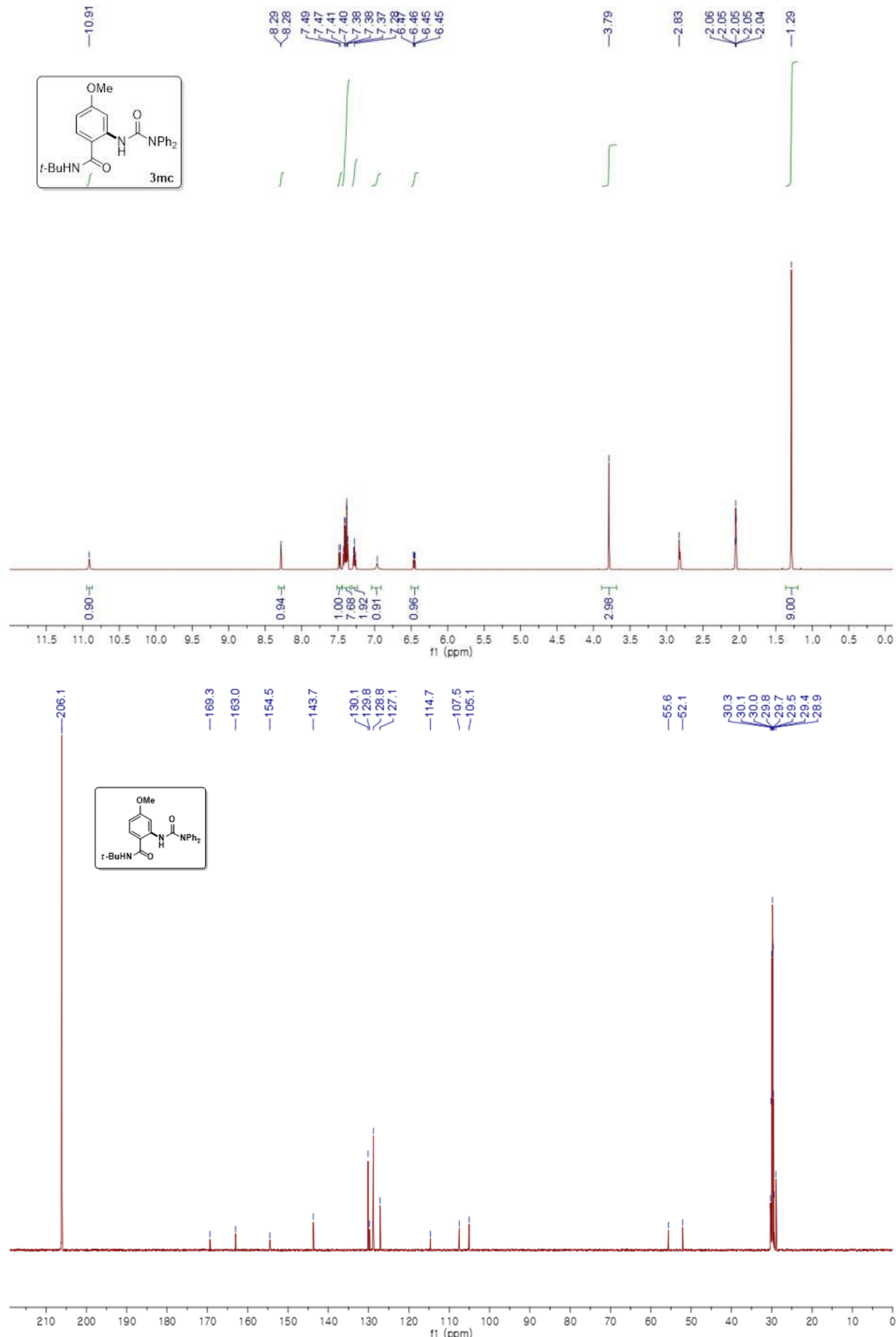


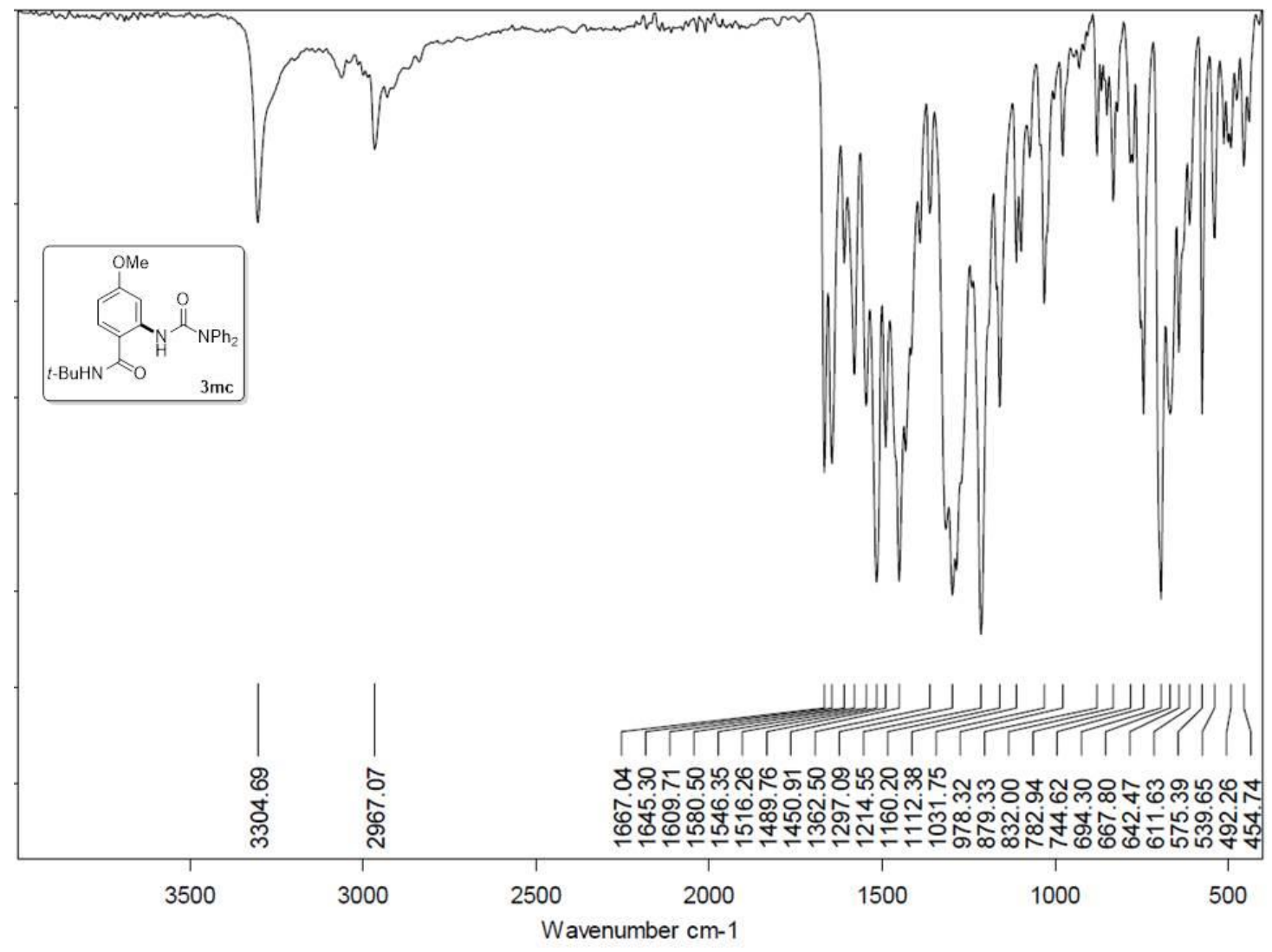


4-Acetyl- $\boldsymbol{N}$-(tert-butyl)-2-(3,3-diphenylureido)benzamide (Table 3, 3nc, ${ }^{1} \mathrm{H}$ NMR-500 MHz \& ${ }^{13} \mathrm{C}$ NMR-125 MHz in Acetone- $\left.d_{6}\right)$

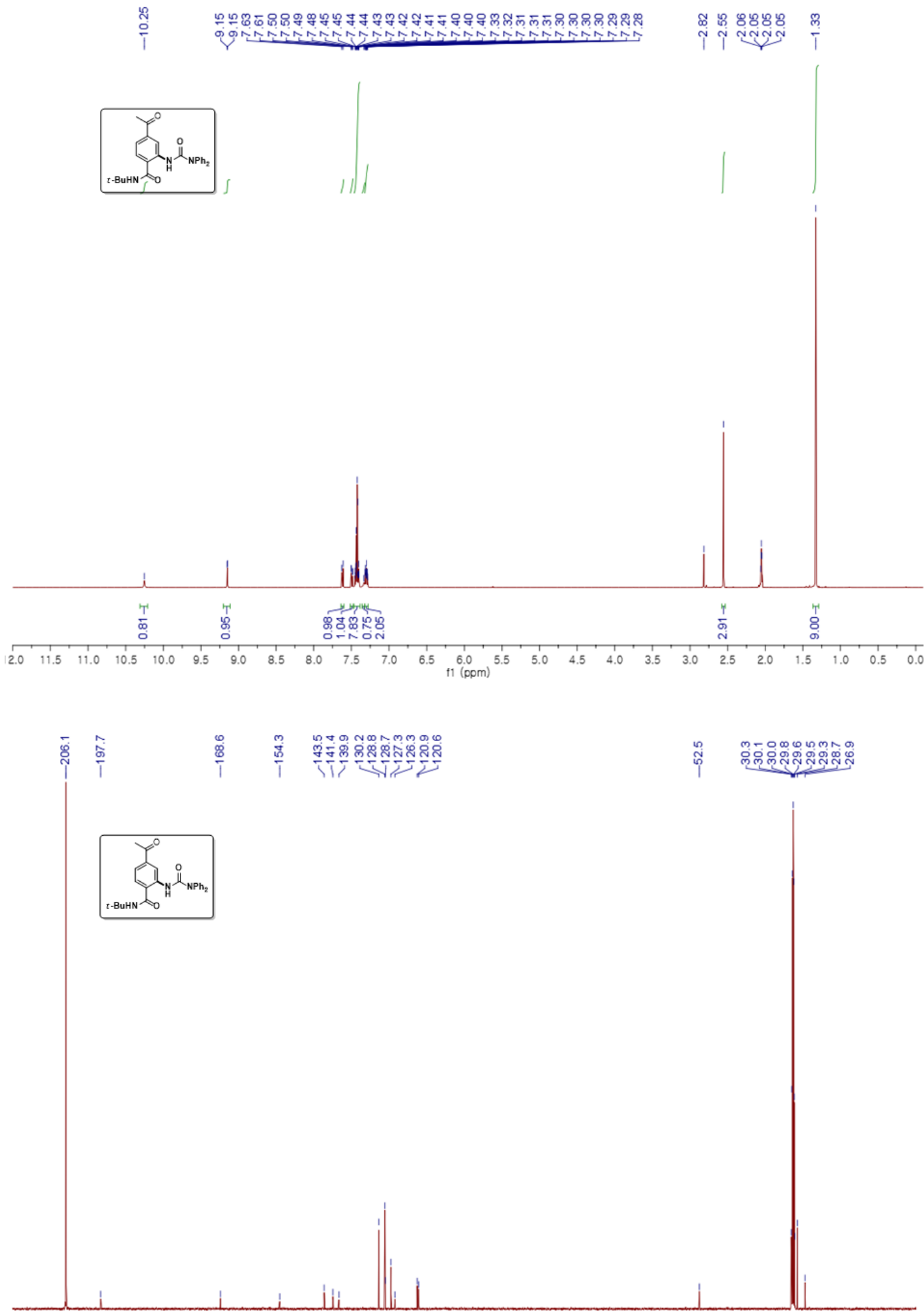

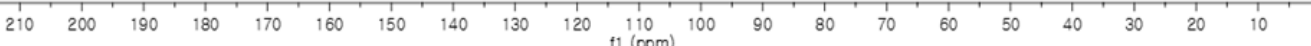




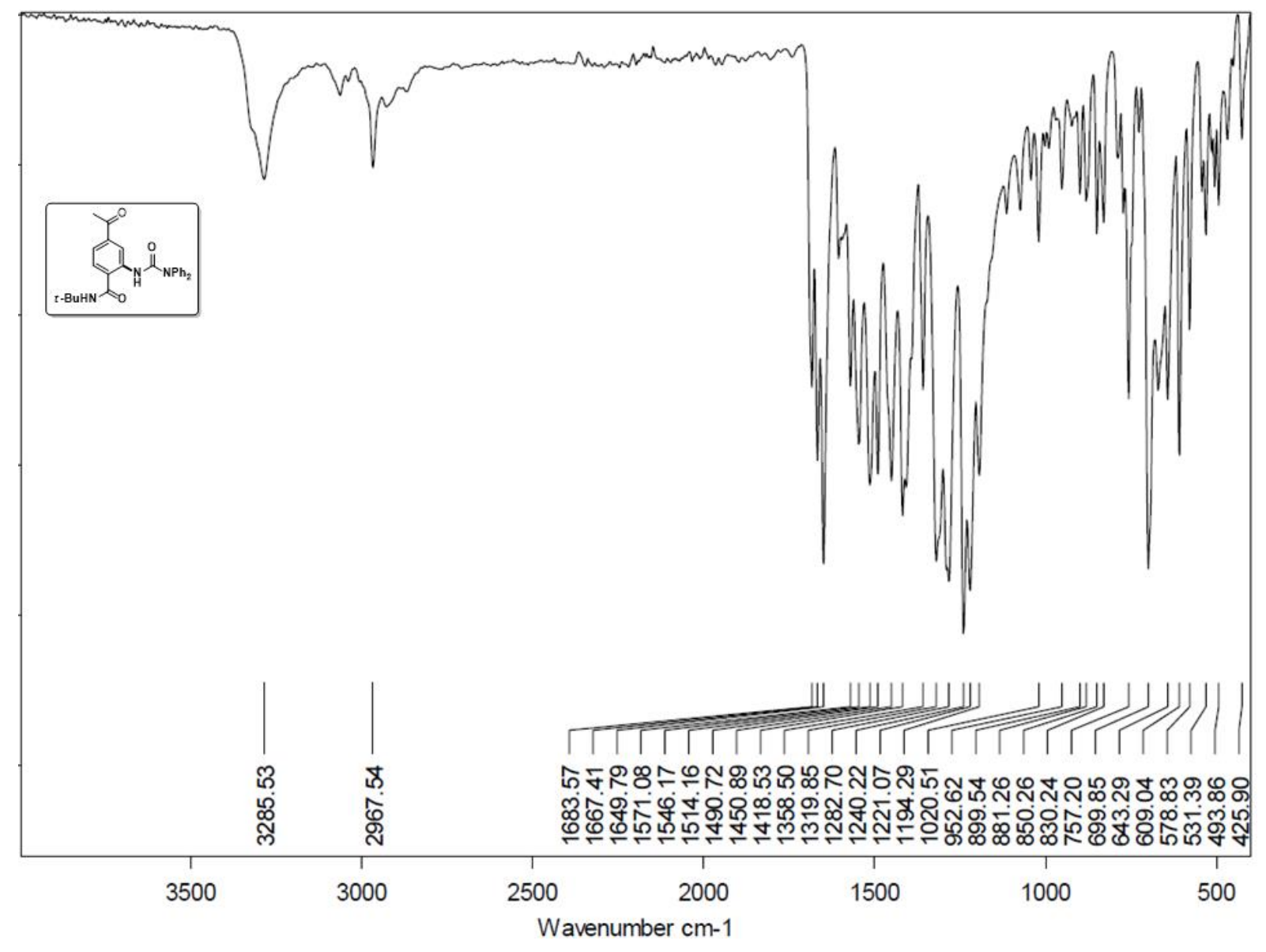


3-(2-Formyl-3-methylphenyl)-1,1-diphenylurea (Scheme 3, 6ac, ${ }^{1} \mathrm{H}$ NMR-500 MHz \& ${ }^{13} \mathrm{C}$ NMR$125 \mathrm{MHz}$ in Acetone- $d_{6}$ )
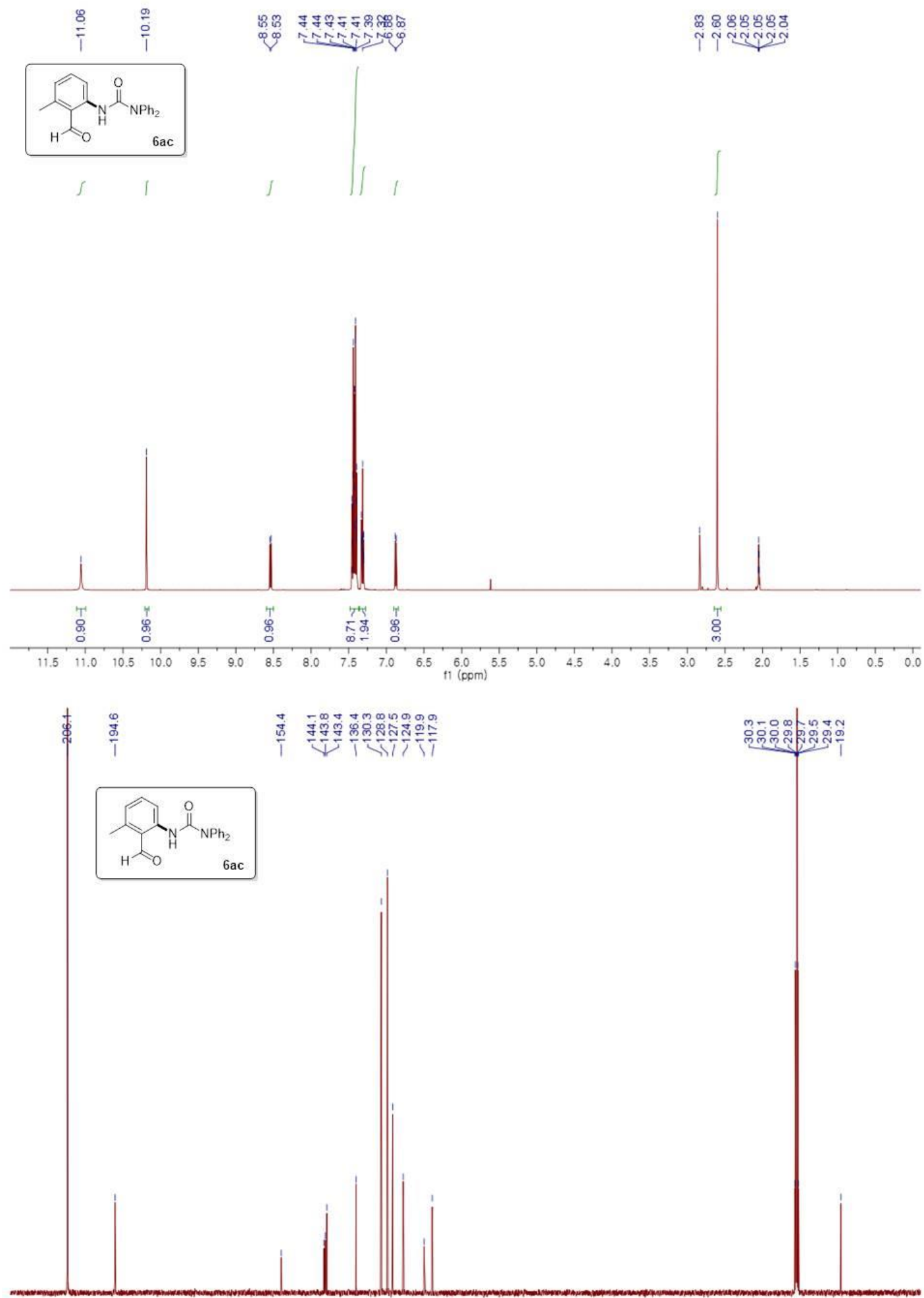

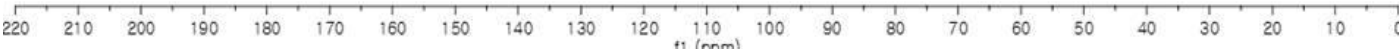




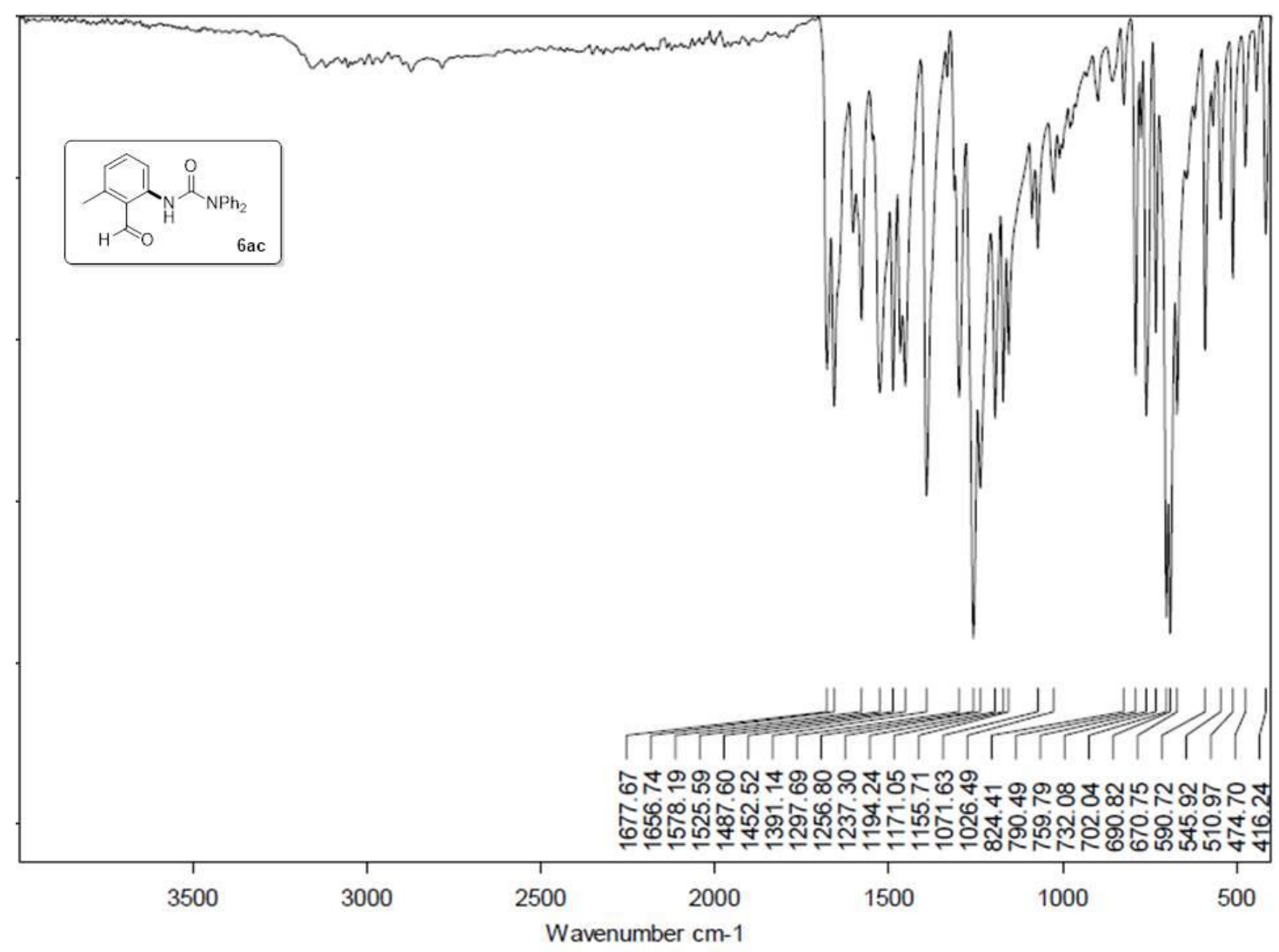

